\title{
GLOBAL CRYSTAL BASES FOR INTEGRABLE MODULES OVER A QUANTUM SYMMETRIC PAIR OF TYPE AIII
}

\author{
HIDEYA WATANABE
}

\begin{abstract}
In this paper, we study basic properties of global -crystal bases for integrable modules over a quantum symmetric pair coideal subalgebra $\mathbf{U}^{3}$ associated to the Satake diagram of type AIII without black nodes. Also, we obtain an intrinsic characterization of the $\mathrm{f}$-crystal bases, whose original definition is artificial.
\end{abstract}

\section{INTRODUCTION}

Let $\mathbf{U}=U_{q}\left(\mathfrak{s l}_{2 r+1}\right)$ be the quantum group over the field $\mathbb{Q}(q)$ of rational functions in one variable $q$, and $\mathbf{U}^{\jmath}$ its coideal subalgebra such that $\left(\mathbf{U}, \mathbf{U}^{\jmath}\right)$ forms a quantum symmetric pair of type AIII in the sense of [Le99]. Bao and Wang BW18a introduced the notion of $\boldsymbol{y}$-canonical bases for the based $\mathbf{U}$-modules. A based $\mathbf{U}$-module is a $\mathbf{U}$-module $M$ with a bar-involution $\psi_{M}$ and a distinguished basis B satisfying certain conditions (see [L10] for the precise definition). One of the key ingredients for the construction of the $\jmath$-canonical bases is the intertwiner (also known as the quasi- $K$-matrix) $\Upsilon$. Using $\Upsilon$, Bao and Wang defined a new involution $\psi_{M}^{J}:=\Upsilon \circ \psi_{M}$ on $M$ which is compatible with the bar-involution $\psi^{\jmath}$ on $\mathbf{U}^{\jmath}$. Then, for each $b \in \mathbf{B}$, there exists a unique $b^{\jmath} \in M$ such that $\psi_{M}^{\jmath}\left(b^{\jmath}\right)=b^{\jmath}$ and $b^{\jmath}-b \in \bigoplus_{\substack{b^{\prime} \in \mathbf{B} \\ b^{\prime} \prec{ }^{\jmath} b}} q \mathbb{Q}[q] b^{\prime}$, where $\preceq^{\jmath}$ is a certain partial order on $\mathbf{B}$. Clearly, $\left\{b^{\jmath} \mid b \in \mathbf{B}\right\}$ is a basis of $M$, which is called the $\jmath$-canonical basis of $(M, \mathbf{B})$.

The multi-parameter version of $\mathbf{U}^{\jmath}$ was considered in BWW18. Thanks to

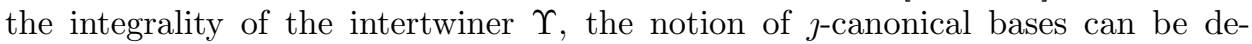
fined analogously. The condition $b^{\jmath}-b \in \underset{\substack{b^{\prime} \in \mathbf{B} \\ b^{\prime} \prec \jmath^{j} b}}{ } q \mathbb{Q}[q] b^{\prime}$ is replaced by $b^{\jmath}-b \in$ $\bigoplus_{b^{\prime} \in \mathbf{B}}\left(p \mathbb{Q}\left[p, q, q^{-1}\right] \oplus q \mathbb{Q}[q]\right) b^{\prime}$. The general theory of the $\jmath$-canonical bases (usually $b^{\prime} \prec^{3} b$

called $\imath$-canonical bases) for the general quantum symmetric pairs was developed in BW18b].

In W17, the author classified all irreducible $\mathbf{U}^{\jmath}$-modules in a category $\mathcal{O}_{\text {int }}^{\jmath}$, which is an analog of the category $\mathcal{O}_{\text {int }}$ of integrable $\mathbf{U}$-modules, and proved that $\mathcal{O}_{\text {int }}^{J}$ is semisimple; the isomorphism classes of irreducible modules in $\mathcal{O}_{\text {int }}^{J}$ are classified by the set $P^{\jmath}$ of bipartitions of length $(r ; r+1)$. When the parameters are in the asymptotic case, to each irreducible module in $\mathcal{O}_{\text {int }}^{J}$, the author associated a local basis, the $\mathrm{f}$-crystal basis, which is an analog of Kashiwara's crystal basis. By the complete reducibility, every object in $\mathcal{O}_{\text {int }}^{J}$ admits a $\jmath$-crystal basis. In particular,

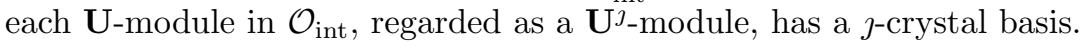

Received by the editors November 16, 2019, and, in revised form, September 21, 2020.

2020 Mathematics Subject Classification. Primary 17B10.

Key words and phrases. Quantum symmetric pair, Hecke algebra, global crystal basis.

This work was supported by JSPS KAKENHI grant number 17J00172. 
It should be noted that the $\mathrm{j}$-crystal basis of a $\mathbf{U}$-module in $\mathcal{O}_{\text {int }}$ is the localized J-canonical basis ( W17, Section 1.3]). To be precise, let $M \in \mathcal{O}_{\text {int }}$ with a canonical basis (or global crystal basis) B. Since $(M, \mathbf{B})$ is a based module, it has a $J_{-}$ canonical basis $\left\{b^{\jmath} \mid b \in \mathbf{B}\right\}$. Set $\mathcal{L}:=\operatorname{Span}_{\mathbf{A}_{0}} \mathbf{B}$, where $\mathbf{A}_{0}:=\{f / g \in \mathbb{Q}(p, q) \mid$ $\left.f, g \in p \mathbb{Q}\left[p, q, q^{-1}\right] \oplus \mathbb{Q}[q], \lim _{q \rightarrow 0}\left(\lim _{p \rightarrow 0} g\right) \neq 0\right\}$. Then, $\mathcal{B}:=\left\{b^{\jmath}+q \mathcal{L} \mid b \in \mathbf{B}\right\}$ is a $\mathbb{Q}$-basis of $\mathcal{L} / q \mathcal{L}$, and $(\mathcal{L}, \mathcal{B})$ forms a $\jmath$-crystal basis of $M$. Hence, $b^{\jmath}+q \mathcal{L}$ can be thought of as the localization of $b^{3}$ at $p=q=0$. Conversely, we may say that the -canonical basis of a based $\mathbf{U}$-module is a globalization of its $\jmath_{\text {-crystal basis. }}$

Here arises a natural question: Does a $\mathbf{U}^{\jmath}$-module in $\mathcal{O}_{\text {int }}^{\jmath}$ that is not a based U-module admit a globalization of its j-crystal basis? One of the main result of this paper gives the affirmative answer to this question.

In our strategy, the multi-parameter $q$-Schur duality between $\mathbf{U}^{\jmath}$ and the Hecke algebra of type $B$ ( $[$ WWW18 $)$, and the irreducibility of the Kazhdan-Lusztig cell representations of the asymptotic multi-parameter Hecke algebra of type $B$ ([BI03]) play key roles. Let us recall the latter objects briefly. Kazhdan and Lusztig [KL79] gave a partition $W=\sqcup_{X \in L(W)} X$ of a Coxeter group $W$ into the left cells; here, $L(W)$ denotes the set of left cells. To each left cell $X \in L(W)$, they associated an $\mathcal{H}$-module $C_{X}^{L}$ which is called the left cell representation corresponding to $X$. The left cell representation $C_{X}^{L}$ is defined to be the quotient of a left ideal $C_{\leq_{L} X}$ of $\mathcal{H}$ spanned by some Kazhdan-Lusztig basis elements by its subspace $C_{<_{L} X}$, which is also spanned by some Kazhdan-Lusztig basis elements. Therefore, $C_{X}^{L}$ has a basis consisting of the images of some Kazhdan-Lusztig basis elements under the canonical map $C_{\leq_{L} X} \rightarrow C_{X}^{L}$. It is known that each left cell representation is irreducible if $W$ is of type $A$. When $W$ is of type $B$, the irreducibility of the left cell representations depends on the choice of the parameters $p, q$. According to [BI03], the left cell representations are irreducible when the parameters are asymptotic.

By the multi-parameter $q$-Schur duality for type $B$, the tensor power $\mathbf{V}^{\otimes d}$ of the vector representation of $\mathbf{U}$ is equipped with a $\left(\mathbf{U}^{\jmath}, \mathbf{H}\right)$-bimodule structure whose irreducible decomposition is multiplicity free, where $\mathbf{H}$ denotes the multi-parameter Hecke algebra of type $B$ over the field $\mathbb{Q}(p, q)$ of rational functions in two variables $p, q$. Then, for each $X \in L(W)$, the left $\mathbf{U}^{\jmath}$-module $\mathbf{V}^{\otimes d} \otimes_{\mathbf{H}} \mathbf{C}_{X}^{L}$ is irreducible, where $\mathbf{C}_{X}^{L}:=\mathbb{Q}(p, q) \otimes_{\mathbb{Z}\left[p^{ \pm 1}, q^{ \pm 1}\right]} C_{X}^{L}$. Every irreducible $\mathbf{U}^{\jmath}$-module can be obtained in this way as $d \geq 1$ varies. The main result of this paper states that the basis of $\mathbf{V}^{\otimes d} \otimes_{\mathbf{H}} \mathbf{C}_{X}^{L}$ induced from the Kazhdan-Lusztig basis of $C_{X}^{L}$ is a globalization of the -crystal basis.

Our approach provides the following characterization of the $\jmath$-crystal bases and its globalization of the finite-dimensional irreducible $\mathbf{U}^{3}$-modules. Let $L \in \mathcal{O}_{\text {int }}^{J}$ be irreducible and $v \in L$ a highest weight vector. Define two symmetric bilinear forms $(\cdot, \cdot)_{1}$ and $(\cdot, \cdot)_{2}$ on $L$ and an involutive anti-linear automorphism $\psi_{L}^{3}$ on $L$ by

$$
\begin{aligned}
& (v, v)_{1}=1,(x m, n)_{1}=\left(m, \sigma^{\jmath}(x) n\right)_{1} \quad \text { for all } x \in \mathbf{U}^{\jmath}, m, n \in L, \\
& (v, v)_{2}=1,(x m, n)_{2}=\left(m, \tau^{\jmath}(x) n\right)_{2} \quad \text { for all } x \in \mathbf{U}^{\jmath}, m, n \in L, \\
& \psi_{L}^{\jmath}(v)=v, \psi_{L}^{\jmath}(x m)=\psi^{\jmath}(x) \psi_{L}^{\jmath}(m) \quad \text { for all } x \in \mathbf{U}^{\jmath}, m \in L,
\end{aligned}
$$

where $\sigma^{\jmath}, \tau^{\jmath}$, and $\psi^{\jmath}$ are automorphisms of $\mathbf{U}^{\jmath}$ defined in Proposition 3.1.1

Theorem A. Let $\boldsymbol{\lambda} \in P^{\jmath}, L(\boldsymbol{\lambda})$ the corresponding irreducible $\mathbf{U}^{\jmath}$-module, $(\mathcal{L}(\boldsymbol{\lambda}), \mathcal{B}(\boldsymbol{\lambda}))$ the $\mathrm{J}$-crystal basis of $L(\boldsymbol{\lambda})$ such that $v+q \mathcal{L}(\boldsymbol{\lambda}) \in \mathcal{B}(\boldsymbol{\lambda})$. Then, there exist $G^{\jmath}(b), b \in \mathcal{B}(\boldsymbol{\lambda})$ satisfying the following. 
(1) $\mathcal{L}(\boldsymbol{\lambda})=\left\{m \in L(\boldsymbol{\lambda}) \mid(m, m)_{2} \in \mathbf{A}_{0}\right\}$.

(2) $\mathcal{B}(\boldsymbol{\lambda})$ forms an orthonormal basis of $\mathcal{L}(\boldsymbol{\lambda}) / q \mathcal{L}(\boldsymbol{\lambda})$ with respect to the symmetric bilinear form induced from $(\cdot, \cdot)_{2}$.

(3) Set $L_{\mathbf{A}}(\boldsymbol{\lambda}):=\operatorname{Span}_{\mathbf{A}}\left\{G^{\jmath}(b) \mid b \in \mathcal{B}(\boldsymbol{\lambda})\right\}$, where $\mathbf{A}:=\mathbb{Q}\left[p, p^{-1}, q, q^{-1}\right]$. Then, the triple $\left(\mathcal{L}(\boldsymbol{\lambda}), L_{\mathbf{A}}(\boldsymbol{\lambda}), \psi_{L}^{J}(\mathcal{L}(\boldsymbol{\lambda}))\right)$ forms a balanced triple with the global basis $\left\{G^{\jmath}(b) \mid b \in \mathcal{B}(\boldsymbol{\lambda})\right\}$.

(4) $L(\boldsymbol{\lambda})$ has the basis dual to $G^{J}(\mathcal{B}(\boldsymbol{\lambda}))$ with respect to $(\cdot, \cdot)_{1}$.

Next, we investigate basic properties of global $\jmath$-crystal basis for not necessarily irreducible $\mathbf{U}^{\jmath}$-modules. Especially, we roughly describe the matrix coefficients of the actions of the generators of $\mathbf{U}^{\jmath}$ with respect to a given global -crystal basis.

We end this paper by proving that the global $\jmath$-crystal basis for a $\mathbf{U}$-module (not $\mathbf{U}^{3}$-module) is compatible with the filtration coming from the dominance order of the bipartitions (see subsection 9.3 for the definition of this filtration). A similar result is well-known for ordinary global crystal bases ([K93], [L10]).

Theorem B. Let $M$ be a $\mathbf{U}$-module with a global j-crystal basis $G^{\jmath}(\mathcal{B})$. Then, for each $\boldsymbol{\lambda} \in P^{\jmath}$, the subquotient $W_{\boldsymbol{\lambda}}(M)$ of $M$ has $\left\{G^{\jmath}(b)+W_{\succ \boldsymbol{\lambda}}(M) \mid I(b)=\boldsymbol{\lambda}\right\}$ as a global $\mathrm{\jmath}$-crystal basis. Moreover, there exists an isomorphism $L(\boldsymbol{\lambda})^{\oplus m_{\boldsymbol{\lambda}}} \rightarrow W_{\boldsymbol{\lambda}}(M)$ which restricts to a bijection $\left\{G^{\jmath}(b) \mid b \in \mathcal{B}(\boldsymbol{\lambda})\right\}^{\oplus m_{\boldsymbol{\lambda}}} \rightarrow\left\{G^{\jmath}(b)+W_{\succ \boldsymbol{\lambda}}(M) \mid I(b)=\right.$ $\boldsymbol{\lambda}\}$, where $m_{\boldsymbol{\lambda}}$ denotes the multiplicity of $L(\boldsymbol{\lambda})$ in $M$.

In particular, if we take $M$ to be an irreducible $\mathbf{U}^{\jmath}$-module, we obtain the following.

Corollary C. Let $\boldsymbol{\lambda} \in P^{\jmath}$. Then, $\left\{G^{\jmath}(b) \mid b \in \mathcal{B}(\boldsymbol{\lambda})\right\}$ is a unique global j-crystal basis for $L(\boldsymbol{\lambda})$.

Finally, we mention that the results above are valid for the quantum symmetric pair $\left(\mathbf{U}, \mathbf{U}^{\imath}\right)$ of type AIV once we define a corresponding category $\mathcal{O}_{\text {int }}^{\imath}$ properly.

This paper is organized as follows. In Section 2, we prepare necessary notations concerning (bi)partitions and Young (bi)tableaux. In Section 3 and 4 , we give a brief review of [W17. In Section [5, we introduce the notion of global $\jmath$-crystal bases, and show that the $f$-canonical bases are examples of them. Sections 6 6 are devoted to proving the existence theorem for the global $\mathrm{j}$-crystal bases of the finitedimensional irreducible $\mathbf{U}^{\jmath}$-modules. After studying basic properties of the global J-crystal bases in Section 9, we finally prove the compatibility of the $\jmath$-crystal bases and the filtration associated to the dominance order of the bipartitions in Section 10.

\section{Notations}

Throughout this paper, we fix a positive integer $r$. For $n \in \frac{1}{2} \mathbb{Z}$, set $\underline{n}:=n-\frac{1}{2}$. Note that $-\underline{n}=-n+\frac{1}{2} \neq \underline{-n}$. We set

$$
I:=\{-r, \ldots,-1,0,1, \ldots, r\}, \quad \mathbb{I}:=\{-\underline{r}, \ldots,-\underline{1}, \underline{1}, \ldots, \underline{r}\}, \quad \mathbb{I}^{\jmath}:=\{1, \ldots, r\} .
$$

A partition of $n \in \mathbb{N}$ of length $l \in \mathbb{N}$ is a nonincreasing sequence $\lambda=\left(\lambda_{1}, \ldots, \lambda_{l}\right)$ of nonnegative integers satisfying $\sum_{i=1}^{l} \lambda_{i}=n$. Let $|\lambda|:=n$ and $\ell(\lambda):=l$, and call them the size and the length of $\lambda$, respectively. We denote by $\operatorname{Par}_{l}(n)$ the set of partitions of $n$ of length $l$.

We often identify a partition with a Young diagram in a usual way. Let $(L, \preceq)$ be a totally ordered set. A semistandard tableau of shape $\lambda \in \operatorname{Par}_{l}(n)$ in letters 
$L$ is a filling of the Young diagram $\lambda$ with elements of $L$, which weakly increases (with respect to the total order $\preceq$ ) from left to right along the rows, and strictly increases from the top to the bottom along the columns.

A bipartition of $n \in \mathbb{N}$ of length $(l ; m) \in \mathbb{N}^{2}$ is an ordered pair $\boldsymbol{\lambda}:=\left(\boldsymbol{\lambda}^{-} ; \boldsymbol{\lambda}^{+}\right)$of partitions such that $\ell\left(\boldsymbol{\lambda}^{-}\right)=l, \ell\left(\boldsymbol{\lambda}^{+}\right)=m$, and $|\boldsymbol{\lambda}|:=\left|\boldsymbol{\lambda}^{-}\right|+\left|\boldsymbol{\lambda}^{+}\right|=n$. We denote by $P_{(l ; m)}(n)$ the set of bipartitions of $n$ of length $(l ; m)$. For totally ordered sets $\left(L^{-}, \preceq^{-}\right)$and $\left(L^{+}, \preceq^{+}\right)$, a semistandard tableau of shape $\boldsymbol{\lambda} \in P_{(l ; m)}(n)$ in letters $\left(L^{-} ; L^{+}\right)$is an ordered pair $\mathbf{T}=\left(T^{-} ; T^{+}\right)$, where $T^{ \pm}$is a semistandard tableau of shape $\boldsymbol{\lambda}^{ \pm}$in letters $L^{ \pm}$.

For partitions $\mu \subset \lambda$, define the skew partition $\lambda / \mu$ in a usual way. For bipartitions $\boldsymbol{\mu} \subset \boldsymbol{\lambda}$ (i.e., $\boldsymbol{\mu}^{-} \subset \boldsymbol{\lambda}^{-}$and $\boldsymbol{\mu}^{+} \subset \boldsymbol{\lambda}^{+}$), define the skew bipartition $\boldsymbol{\lambda} / \boldsymbol{\mu}$ to be $\left(\boldsymbol{\lambda}^{-} / \boldsymbol{\mu}^{-} ; \boldsymbol{\lambda}^{+} / \boldsymbol{\mu}^{+}\right)$. A skew partition $\lambda / \mu$ is said to be a horizontal strip if each column of $\lambda / \mu$ contains at most one box. We say that a skew bipartition $\boldsymbol{\lambda} / \boldsymbol{\mu}$ is a horizontal strip if $\boldsymbol{\lambda}^{ \pm} / \boldsymbol{\mu}^{ \pm}$are.

Set

- $P(n)=P_{r}(n):=\operatorname{Par}_{2 r+1}(n)$ : the set of partitions of $n$ of length $2 r+1$.

- $P=P_{r}:=\bigsqcup_{n \in \mathbb{N}} P(n)$ : the set of partitions of length $2 r+1$.

- $\operatorname{Par}_{l}:=\bigsqcup_{n \in \mathbb{N}} \operatorname{Par}_{l}(n)$ : the set of partitions of length $l$.

- $P^{\jmath}(n)=P_{r}^{J}(n):=P_{(r+1 ; r)}(n)$ : the set of bipartitions of $n$ of length $(r+1 ; r)$.

- $P^{\jmath}=P_{r}^{\jmath}:=\bigsqcup_{n \in \mathbb{N}} P^{\jmath}(n)$ : the set of bipartitions of length $(r+1 ; r)$.

- $\operatorname{SST}(\lambda)$ : the set of semistandard tableaux of shape $\lambda \in P(n)$ in letters $I$.

- $\operatorname{SST}(\boldsymbol{\lambda})$ : the set of semistandard tableaux of shape $\boldsymbol{\lambda} \in P^{\jmath}(n)$ in letters $\left(I \backslash \mathbb{I}^{\jmath} ; \mathbb{I}^{J}\right)$ with total orders $0 \prec^{-}-1 \prec^{-} \ldots \prec^{-}-r$ and $1 \prec^{+} \ldots \prec^{+} r$.

For $\boldsymbol{\lambda} \in P^{3}$, we refer the $i$-th row of $\boldsymbol{\lambda}^{-}$to as the $-(i-1)$-th row of $\boldsymbol{\lambda}$, and the $j$-th row of $\boldsymbol{\lambda}^{+}$to as the $j$-th row of $\boldsymbol{\lambda}$. Also, for $i \in I$, set $\boldsymbol{\lambda}_{i}$ to be the length of the $i$-th row of $\boldsymbol{\lambda}$, i.e.,

$$
\boldsymbol{\lambda}_{i}:= \begin{cases}\boldsymbol{\lambda}_{-i+1}^{-} & \text {if } i \leq 0, \\ \boldsymbol{\lambda}_{i}^{+} & \text {if } i>0 .\end{cases}
$$

For $i \in \mathbb{I}^{\jmath}$, set $\boldsymbol{\lambda} \downarrow_{i}:=\left(\boldsymbol{\lambda}_{0}, \boldsymbol{\lambda}_{-1}, \ldots, \boldsymbol{\lambda}_{-i} ; \boldsymbol{\lambda}_{1}, \ldots, \boldsymbol{\lambda}_{i}\right) \in P_{i}^{\jmath}$.

For $\mathbf{T} \in \operatorname{SST}(\boldsymbol{\lambda})$ and $i \in \mathbb{I}^{\jmath}$, set $\mathbf{T} \downarrow_{i}$ to be the semistandard tableau obtained from $\mathbf{T}$ by deleting the boxes whose entries are less than $-i$ or greater than $i$.

For each $\boldsymbol{\lambda} \in P^{3}$, let $\mathbf{T}_{\boldsymbol{\lambda}} \in \operatorname{SST}(\boldsymbol{\lambda})$ be the unique semistandard tableau of shape $\boldsymbol{\lambda}$ whose entries in the $i$-th row are $i$. Note that we have $\mathbf{T}_{\boldsymbol{\lambda}} \downarrow_{i}=\mathbf{T}_{\boldsymbol{\lambda}_{\downarrow_{i}}}$. For $\mathbf{T} \in \operatorname{SST}(\boldsymbol{\lambda})$ and $i \in I$, set $\mathbf{T}(i)$ to be the number of boxes of $\mathbf{T}$ whose entries are $i$.

\section{Definition 2.0.1.}

(1) $\preceq$ is a partial order (called the dominance order) on $\operatorname{Par}_{l}$ defined as follows. For $\lambda, \mu \in \operatorname{Par}_{l}$, we have $\lambda \preceq \mu$ if

(a) $|\lambda|=|\mu|$ and

(b) $\sum_{i=1}^{j} \lambda_{i} \leq \sum_{i=1}^{j} \mu_{i}$ for all $1 \leq j \leq l$.

(2) $\preceq$ is a partial order (also called the dominance order) on $P^{\jmath}$ defined as follows. For $\boldsymbol{\lambda}, \boldsymbol{\mu} \in P^{\jmath}$, we have $\boldsymbol{\lambda} \preceq \boldsymbol{\mu}$ if

(a) $|\boldsymbol{\lambda}|=|\boldsymbol{\mu}|$,

(b) $\sum_{i=0}^{j} \boldsymbol{\lambda}_{-i} \leq \sum_{i=0}^{j} \boldsymbol{\mu}_{-i}$ for all $0 \leq j \leq r$, and

(c) $\left|\boldsymbol{\lambda}^{-}\right|+\sum_{i=1}^{j} \boldsymbol{\lambda}_{i} \leq\left|\boldsymbol{\mu}^{-}\right|+\sum_{i=1}^{j} \boldsymbol{\mu}_{i}$ for all $1 \leq j \leq r$. 
(3) $\unlhd$ is a partial order on $P^{\jmath}$ defined as follows. For $\boldsymbol{\lambda}, \boldsymbol{\mu} \in P^{\jmath}$, we have $\boldsymbol{\lambda} \unlhd \boldsymbol{\mu}$ if $\boldsymbol{\lambda}^{-} \preceq \boldsymbol{\mu}^{-}$(dominance order on $\operatorname{Par}_{r+1}$ ) and $\boldsymbol{\lambda}^{+} \preceq \boldsymbol{\mu}^{+}$(dominance order on $\left.\operatorname{Par}_{r}\right)$.

Clearly, $\boldsymbol{\lambda} \unlhd \boldsymbol{\mu}$ implies $\boldsymbol{\lambda} \preceq \boldsymbol{\mu}$.

\section{Representation theory of $\mathbf{U}^{3}$}

Let $p$ and $q$ be independent indeterminates.

3.1. Definition of $\mathbf{U}^{\jmath}$. Let $\Lambda$ be the free $\mathbb{Z}$-module with a free basis $\left\{\epsilon_{i} \mid i \in I\right\}$, and with a symmetric bilinear form $(\cdot, \cdot)$ defined by $\left(\epsilon_{i}, \epsilon_{j}\right)=\delta_{i, j}$. For $i \in \mathbb{I}$, set

$$
\alpha_{i}:=\epsilon_{\underline{i}}-\epsilon_{\underline{i+1}}, \quad Q:=\sum_{i \in \mathbb{I}} \mathbb{Z} \alpha_{i}, \quad Q_{+}:=\sum_{i \in \mathbb{I}} \mathbb{Z}_{\geq 0} \alpha_{i}
$$

For $\lambda, \mu \in \Lambda$, we write $\mu \leq \lambda$ if $\lambda-\mu \in Q_{+}$. This defines a partial order on $\Lambda$.

The quantum group $\mathbf{U}=\mathbf{U}_{2 r+1}=U_{q}\left(\mathfrak{s l}_{2 r+1}\right)$ of type $A_{2 r}$ is an associative algebra over $\mathbb{Q}(p, q)$ with generators $E_{i}, F_{i}, K_{i}^{ \pm 1}, i \in \mathbb{I}$ subject to the following relations: For $i, j \in \mathbb{I}$,

$$
\begin{aligned}
& K_{i} K_{i}^{-1}=K_{i}^{-1} K_{i}=1, \\
& K_{i} K_{j}=K_{j} K_{i}, \\
& K_{i} E_{j} K_{i}^{-1}=q^{\left(\alpha_{i}, \alpha_{j}\right)} E_{i}, \\
& K_{i} F_{j} K_{i}^{-1}=q^{-\left(\alpha_{i}, \alpha_{j}\right)} F_{i}, \\
& E_{i} F_{j}-F_{j} E_{i}=\delta_{i, j} \frac{K_{i}-K_{i}^{-1}}{q-q^{-1}}, \\
& E_{i}^{2} E_{j}-\left(q+q^{-1}\right) E_{i} E_{j} E_{i}+E_{j} E_{i}^{2}=0 \quad \text { if }|i-j|=1, \\
& F_{i}^{2} F_{j}-\left(q+q^{-1}\right) F_{i} F_{j} F_{i}+F_{j} F_{i}^{2}=0 \quad \text { if }|i-j|=1, \\
& E_{i} E_{j}-E_{j} E_{i}=0 \quad \text { if }|i-j|>1, \\
& F_{i} F_{j}-F_{j} F_{i}=0 \quad \text { if }|i-j|>1 .
\end{aligned}
$$

In this paper, we use the comultiplication $\Delta$ of $\mathbf{U}$ given by

$$
\begin{aligned}
& \Delta\left(K_{i}^{ \pm 1}\right)=K_{i}^{ \pm 1} \otimes K_{i}^{ \pm 1}, \quad \Delta\left(E_{i}\right)=1 \otimes E_{i}+E_{i} \otimes K_{i}^{-1}, \\
& \Delta\left(F_{i}\right)=F_{i} \otimes 1+K_{i} \otimes F_{i} \quad i \in \mathbb{I} .
\end{aligned}
$$

Let $\left(\mathbf{U}, \mathbf{U}^{\jmath}\right)$ denote the quantum symmetric pair over $\mathbb{Q}(p, q)$ of type AIII without black nodes, that is, $\mathbf{U}^{\jmath}=\mathbf{U}_{r}^{\jmath}$ is the subalgebra of $\mathbf{U}$ generated by

$$
\begin{aligned}
& k_{i}^{ \pm 1}:=\left(K_{\underline{i}} K_{-\underline{i}}\right)^{ \pm 1}, \\
& e_{i}:=E_{\underline{i}}+p^{-\delta_{i, 1}} F_{-\underline{i}} K_{\underline{i}}^{-1}, \\
& f_{i}:=E_{-\underline{i}}+p^{\delta_{i, 1}} K_{-\underline{i}}^{-1} F_{\underline{i}}, \quad i \in \mathbb{I}^{\jmath} .
\end{aligned}
$$


The $\mathbf{U}^{\jmath}$ has the following defining relations ([Le99], see also [BW18a], BWW18]): For $i, j \in \mathbb{I}^{\prime}$,

$$
\begin{aligned}
& k_{i} k_{i}^{-1}=k_{i}^{-1} k_{i}=1, \\
& k_{i} k_{j}=k_{j} k_{i}, \\
& k_{i} e_{j} k_{i}^{-1}=q^{\left(\alpha_{\underline{i}}-\alpha_{-\underline{i}}, \alpha_{\underline{j}}\right)} e_{j}, \\
& k_{i} f_{j} k_{i}^{-1}=q^{-\left(\alpha_{\underline{i}}-\alpha_{-\underline{i}}, \alpha_{\underline{j}}\right)} f_{j}, \\
& e_{i} f_{j}-f_{j} e_{i}=\delta_{i, j} \frac{k_{i}-k_{i}^{-1}}{q-q^{-1}} \quad \text { if }(i, j) \neq(1,1), \\
& e_{i}^{2} e_{j}-\left(q+q^{-1}\right) e_{i} e_{j} e_{i}+e_{j} e_{i}^{2}=0 \quad \text { if }|i-j|=1, \\
& f_{i}^{2} f_{j}-\left(q+q^{-1}\right) f_{i} f_{j} f_{i}+f_{j} f_{i}^{2}=0 \quad \text { if }|i-j|=1, \\
& e_{i} e_{j}-e_{j} e_{i}=0 \quad \text { if }|i-j|>1, \\
& f_{i} f_{j}-f_{j} f_{i}=0 \quad \text { if }|i-j|>1, \\
& e_{1}^{2} f_{1}-\left(q+q^{-1}\right) e_{1} f_{1} e_{1}+f_{1} e_{1}^{2}=-\left(q+q^{-1}\right) e_{1}\left(p q k_{1}+p^{-1} q^{-1} k_{1}^{-1}\right), \\
& f_{1}^{2} e_{1}-\left(q+q^{-1}\right) f_{1} e_{1} f_{1}+e_{1} f_{1}^{2}=-\left(q+q^{-1}\right)\left(p q k_{1}+p^{-1} q^{-1} k_{1}^{-1}\right) f_{1} .
\end{aligned}
$$

\section{Proposition 3.1.1.}

(1) BW18a, Lemma 6.1 (3)]. There exists a unique $\mathbb{Q}$-algebra automorphism $\psi^{\jmath}$ of $\mathbf{U}^{\jmath}$ which maps $e_{i}, f_{i}, k_{i}, p, q$ to $e_{i}, f_{i}, k_{i}^{-1}, p^{-1}, q^{-1}$, respectively.

(2) There exists a unique $\mathbb{Q}(p, q)$-algebra anti-automorphism $\sigma^{\jmath}$ of $\mathbf{U}^{3}$ which maps $e_{i}, f_{i}, k_{i}$ to $f_{i}, e_{i}, k_{i}$, respectively.

(3) BW18b, Proposition 4.6]. There exists a unique $\mathbb{Q}(p, q)$-algebra anti-automorphism $\tau^{\jmath}$ of $\mathbf{U}^{\jmath}$ which maps $e_{i}, f_{i}, k_{i}$ to $p^{-\delta_{i, 1}} q^{-1} k_{i}^{-1} f_{i}, p^{\delta_{i, 1}} q e_{i} k_{i}, k_{i}$, respectively.

Proof. It suffices to show that the images of the generators of $\mathbf{U}^{3}$ satisfy the defining relations of $\mathbf{U}^{\jmath}$; it is straightforward.

Remark 3.1.2. We have similar automorphisms on $\mathbf{U}$ :

(1) There exists a unique $\mathbb{Q}$-algebra automorphism $\psi$ of $\mathbf{U}$ which maps $E_{i}, F_{i}$, $K_{i}, p, q$ to $E_{i}, F_{i}, K_{i}^{-1}, p^{-1}, q^{-1}$, respectively.

(2) There exists a unique $\mathbb{Q}(p, q)$-algebra anti-automorphism $\sigma$ of $\mathbf{U}^{3}$ which maps $E_{i}, F_{i}, K_{i}$ to $F_{i}, E_{i}, K_{i}$, respectively.

(3) There exists a unique $\mathbb{Q}(p, q)$-algebra anti-automorphism $\tau$ of $\mathbf{U}^{\jmath}$ which maps $E_{i}, F_{i}, K_{i}$ to $q F_{i} K_{i}^{-1}, q^{-1} K_{i} E_{i}, K_{i}$, respectively.

Note that $\tau^{\jmath}$ is the restriction of $\tau$ [BW18b, Proposition 4.6], while the others are not.

Let $\mathbf{U}(\mathfrak{l})$ denote the subalgebra of $\mathbf{U}$ generated by $E_{i}, F_{i}, K_{j}^{ \pm 1}, i \in \mathbb{I} \backslash\{\underline{1}\}, j \in \mathbb{I}$. Note that we have $e_{i}, f_{i}, k_{j} \in \mathbf{U}(\mathfrak{l})$ for all $i \in \mathbb{I}^{\jmath} \backslash\{1\}, j \in \mathbb{I}^{\jmath}$. Note that $\mathbf{U}(\mathfrak{l})$ is the quantum group of type $A_{r} \times A_{r-1}$ with weight lattice $\Lambda$.

3.2. Category $\mathcal{O}_{\text {int }}^{J}$. Let us extend the bilinear form $(\cdot, \cdot)$ on $\Lambda$ to $\Lambda_{\mathbb{R}}:=\mathbb{R} \otimes_{\mathbb{Z}} \Lambda$. Set $\beta_{i}:=\alpha_{\underline{i}}-\alpha_{-\underline{i}}, i \in \mathbb{I}^{\jmath}$, and $J:=\left\{\lambda \in \Lambda_{\mathbb{R}} \mid\left(\beta_{i}, \lambda\right)=0\right.$ for all $\left.i \in \mathbb{I}^{\jmath}\right\}$. Then, the induced bilinear form $(\cdot, \cdot):\left(\sum_{i \in \mathbb{I} J} \mathbb{R} \beta_{i}\right) \times\left(\Lambda_{\mathbb{R}} / J\right) \rightarrow \mathbb{R}$ denoted by the same symbol is nondegenerate. Let $\delta_{j} \in \Lambda_{\mathbb{R}} / J$ be such that $\left(\beta_{i}, \delta_{j}\right)=\delta_{i, j}$ for all $i, j \in \mathbb{I}^{\jmath}$. 
Set $\Lambda^{\jmath}:=\sum_{i \in \mathbb{I}^{\jmath}} \mathbb{Z} \delta_{i}$. Let $\gamma_{i}:=\alpha_{\underline{i}}+J \in \Lambda^{\jmath}, i \in \mathbb{I}^{\jmath}$, and $Q_{+}^{\jmath}:=\sum_{i \in \mathbb{I}^{\jmath}} \mathbb{Z}_{\geq 0} \gamma_{i} \subset \Lambda^{\jmath}$. For $\lambda, \mu \in \Lambda^{\jmath}$, we write $\mu \leq^{\jmath} \lambda$ if $\bar{\lambda}-\mu \in Q_{+}^{J}$. This defines a partial order on $\Lambda^{\jmath}$.

For a $\mathbf{U}^{\jmath}$-module $M$ and $\lambda \in \Lambda^{\jmath}$, we call $M_{\lambda}:=\left\{m \in M \mid k_{i} m=q^{\left(\beta_{i}, \lambda\right)} m\right.$ for all $\left.i \in \mathbb{I}^{\jmath}\right\}$ the weight space of $M$ of weight $\lambda$. The category $\mathcal{O}_{\text {int }}^{J}$ is the full subcategory of the category of all $\mathbf{U}^{\jmath}$-modules consisting of $\mathbf{U}^{\jmath}$-modules $M$ satisfying the following:

- $M$ has a weight space decomposition, i.e., $M=\bigoplus_{\lambda \in \Lambda^{J}} M_{\lambda}$.

- Each weight space of $M$ is finite-dimensional.

- There exist $\mu_{1}, \ldots, \mu_{l} \in \Lambda^{\jmath}$ such that if $M_{\lambda} \neq 0$, then $\lambda \leq{ }^{\jmath} \mu_{i}$ for some $i=1, \ldots, l$.

- The $f_{i}$ 's act on $M$ locally nilpotently.

Theorem 3.2.1 ([W17]). The following hold:

(1) W17, Theorem 4.4.3]. $\mathcal{O}_{\mathrm{int}}^{\mathrm{J}}$ is semisimple.

(2) [W17, Corollary 7.6.3, 7.6.4]. Each irreducible $\mathbf{U}^{3}$-module in $\mathcal{O}_{\text {int }}^{J}$ is isomorphic to the irreducible highest weight module $L(\boldsymbol{\lambda})$ with highest weight $\boldsymbol{\lambda}$ (in the sense of [W17]) for some $\boldsymbol{\lambda} \in P^{J}$.

(3) For $\boldsymbol{\lambda}, \boldsymbol{\mu} \in P^{3}$, we have $L(\boldsymbol{\lambda}) \simeq L(\boldsymbol{\mu})$ if and only if $\boldsymbol{\lambda}_{i}-\boldsymbol{\mu}_{i}$ is constant as $i$ runs through $-r, \ldots, r$.

Remark 3.2.2. The last statement follows from the definition of $L(\boldsymbol{\lambda})$.

For each $\boldsymbol{\lambda} \in P^{3}$, let $\mathrm{wt}^{\jmath}(\boldsymbol{\lambda}) \in \Lambda^{\jmath}$ denote the weight of a highest weight vector of $L(\boldsymbol{\lambda})$, namely,

$$
\mathrm{wt}^{\jmath}(\boldsymbol{\lambda}):=\sum_{i \in \mathbb{I}^{\jmath}}\left(\boldsymbol{\lambda}_{i-1}-\boldsymbol{\lambda}_{i}+\boldsymbol{\lambda}_{-(i-1)}-\boldsymbol{\lambda}_{-i}\right) \delta_{i} .
$$

Remark 3.2.3. There is an algebra $\mathbf{U}^{\imath}$ that is closely related to $\mathbf{U}^{\jmath}$ (see [BW18a]). It is a coideal subalgebra of $U_{q}\left(\mathfrak{s l}_{2 r}\right)$ such that the pair $\left(U_{q}\left(\mathfrak{s l}_{2 r}\right), \mathbf{U}^{\imath}\right)$ forms a quantum symmetric pair of type AIV. As mentioned in the introduction part, the results in this paper have counterparts for $\mathbf{U}^{\imath}$. The arguments are parallel except the definition of the category $\mathcal{O}_{\text {int }}^{\imath}$, which is the $\mathbf{U}^{\imath}$-analog of the category $\mathcal{O}_{\text {int }}^{\jmath}$. The main difference between $\mathbf{U}^{\imath}$ and $\mathbf{U}^{\imath}$ is that $\mathbf{U}^{\imath}$ has a distinguished generator $t$. In the definition of $\mathcal{O}_{\text {int }}^{\imath}$, we have to add the constraint that $t$ acts on each $M \in \mathcal{O}_{\text {int }}^{\imath}$ diagonally with eigenvalues of the form $\frac{p q^{a}-p^{-1} q^{-a}}{q-q^{-1}}, a \in \mathbb{Z}$. Then, $\mathcal{O}_{\text {int }}^{\imath}$ becomes semisimple, and the isoclasses of irreducible modules in $\mathcal{O}_{\text {int }}^{\imath}$ is parametrized by the set of bipartitions of length $(r ; r)$.

\section{Crystal basis theory}

4.1. Crystal bases. The notion of crystal bases (or local bases at $q=0$ ) for integrable modules over quantum groups was introduced independently by Kashiwara and Lusztig in different ways ([K90, L90a $)$. Although we will not review the detail, we formulate here some notations concerning the crystal bases. Let $\mathcal{O}_{\text {int }}$ denote the full subcategory of the BGG-category $\mathcal{O}$ for $\mathbf{U}$ consisting of the integrable modules. Let $\widetilde{E}_{i}, \widetilde{F}_{i}, i \in \mathbb{I}$ denote the Kashiwara operators. Let $M \in \mathcal{O}_{\text {int }},(\mathcal{L}, \mathcal{B})$ be its crystal basis. For $b \in \mathcal{B}$ and $i \in \mathbb{I}$, set

$$
\varepsilon_{i}(b):=\max \left\{n \mid \widetilde{E}_{i}^{n} b \neq 0\right\}, \quad \varphi_{i}(b):=\max \left\{n \mid \widetilde{F}_{i}^{n} b \neq 0\right\} .
$$

Also, $\operatorname{wt}(b) \in \Lambda$ denotes the weight of $b$. 
Recall that, for each $\lambda \in P$, the irreducible module $L(\lambda)$ has a unique crystal basis $(\mathcal{L}(\lambda), \mathcal{B}(\lambda))$, which is identical to $\operatorname{SST}(\lambda)$. For each $M \in \mathcal{O}_{\text {int }}$ with a crystal basis $(\mathcal{L}, \mathcal{B})$, we have a unique irreducible decomposition $\mathcal{B}=\bigsqcup_{i=1}^{l} \mathcal{B}_{i}$, where $\mathcal{B}_{i} \simeq$ $\mathcal{B}\left(\lambda_{i}\right)$ for some $\lambda_{i} \in P$. By retaking $\lambda_{i}$ 's if necessary, we may assume that $\left|\lambda_{i}\right|-$ $\left|\lambda_{j}\right|<2 r+1$ for all $i, j \in\{1, \ldots, l\}$, and that there exists $i$ such that $\left(\lambda_{i}\right)_{2 r+1}=0$. Then, $\lambda_{i}$ 's are uniquely determined; we set $P(M)=P_{r}(M):=\left\{\lambda_{1}, \ldots, \lambda_{l}\right\}$. For $b \in \mathcal{B}$, we define $I(b)=I_{r}(b) \in P(M)$ to be $\lambda_{i}$ if $b \in \mathcal{B}_{i}$. Also let $C(b)=C_{r}(b) \subset$ $\mathcal{B}$ denote the connected component of $\mathcal{B}$ containing $b$. Furthermore, if we write $b=\widetilde{F}_{i_{1}} \cdots \widetilde{F}_{i_{l}} b_{0}$ for some $i_{1}, \ldots, i_{l} \in \mathbb{I}$, where $b_{0}$ denotes the highest weight vector in $C(b)$, then define $T_{b} \in \operatorname{SST}(I(b))$ by $T_{b}:=\widetilde{F}_{i_{1}} \cdots \widetilde{F}_{i_{l}} T_{0}$, where $T_{0} \in \operatorname{SST}(I(b))$ corresponding to $b_{0} \in C(b)=\mathcal{B}(I(b))$.

4.2. $\jmath$-crystal bases. In W17, the notion of $\jmath$-crystal bases was introduced. Let us recall some properties briefly.

Set $\mathbf{A}:=\mathbb{Q}\left[p, p^{-1}, q, q^{-1}\right]$. We denote by $\mathbf{A}_{0}$ the subring of $\mathbb{Q}(p, q)$ consisting of all elements of the form $f / g$ with $f, g \in p \mathbb{Q}\left[p, q, q^{-1}\right] \oplus \mathbb{Q}[q], \lim _{q \rightarrow 0}\left(\lim _{p \rightarrow 0} g\right) \neq 0$. Let $\overline{\mathbb{I}^{\jmath}}:=\mathbb{I}^{\jmath} \sqcup\left\{2^{\prime}, \ldots, r^{\prime}\right\}$. The Kashiwara operators are denoted by $\widetilde{e}_{i}$ and $\widetilde{f}_{i}, i \in \overline{\mathbb{I}^{\jmath}}$.

The following are basic results for the crystal basis theory of $\mathbf{U}^{\jmath}$.

Theorem 4.2.1 ([W17, Theorem 7.7.3]). Let $\boldsymbol{\lambda} \in P^{3}, v_{\boldsymbol{\lambda}} \in L(\boldsymbol{\lambda})$ be a highest weight vector. Set

$$
\begin{aligned}
& \mathcal{L}(\boldsymbol{\lambda}):=\operatorname{Span}_{\mathbf{A}_{0}}\left\{\tilde{f}_{i_{1}} \cdots \widetilde{f}_{i_{l}} v_{\boldsymbol{\lambda}} \mid l \in \mathbb{Z}_{\geq 0}, i_{1}, \ldots, i_{l} \in \overline{\mathbb{I}^{\jmath}}\right\} \\
& \mathcal{B}(\boldsymbol{\lambda}):=\left\{\widetilde{f}_{i_{1}} \cdots \widetilde{f}_{i_{l}} v_{\boldsymbol{\lambda}}+q \mathcal{L}(\boldsymbol{\lambda}) \mid l \in \mathbb{Z}_{\geq 0}, i_{1}, \ldots, i_{l} \in \overline{\mathbb{I}^{\jmath}}\right\} \backslash\{0\} .
\end{aligned}
$$

Then, $(\mathcal{L}(\boldsymbol{\lambda}), \mathcal{B}(\boldsymbol{\lambda}))$ is a unique $\mathrm{\jmath}$-crystal basis of $L(\boldsymbol{\lambda})$. Moreover, $\mathcal{B}(\boldsymbol{\lambda})$ is identical to $\operatorname{SST}(\boldsymbol{\lambda}) ; v_{\boldsymbol{\lambda}}+q \mathcal{L}(\boldsymbol{\lambda}) \in \mathcal{B}(\boldsymbol{\lambda})$ corresponds to $\mathbf{T}_{\boldsymbol{\lambda}} \in \operatorname{SST}(\boldsymbol{\lambda})$.

Theorem 4.2.2. Suppose that $M \in \mathcal{O}_{\text {int }}$ has a crystal basis $(\mathcal{L}, \mathcal{B})$. Then, as a $\mathbf{U}^{3}$-module, $M$ has a $\mathrm{j}$-crystal basis whose underlying sets are equal to $(\mathcal{L}, \mathcal{B})$.

Proof. This is an easy consequence of [W17, Corollary 7.7.4].

Let $M \in \mathcal{O}_{\text {int }}^{\jmath}$ with a $\jmath$-crystal basis $(\mathcal{L}, \mathcal{B})$. For each $b \in \mathcal{B}$ and $i \in \overline{\mathbb{I}^{\jmath}}$, define $\varepsilon_{i}(b), \varphi_{i}(b), \mathrm{wt}^{\jmath}(b) \in \Lambda^{\jmath}, P^{\jmath}(M)=P_{r}^{\jmath}(M) \subset P^{\jmath}, I^{\jmath}(b)=I_{r}^{\jmath}(b) \in P^{\jmath}(M), C^{\jmath}(b)=$ $C_{r}^{\jmath}(b) \subset \mathcal{B}$, and $T_{b}^{\jmath} \in \operatorname{SST}\left(I^{\jmath}(b)\right)$ in a similar way to Section 4.1 .

\section{Global Bases}

5.1. Balanced triples. Let $\div$ be the $\mathbb{Q}$-linear automorphism of $\mathbb{Q}(p, q)$ sending $p$ and $q$ to $p^{-1}$ and $q^{-1}$, respectively. Set $\mathbf{A}_{\infty}:=\overline{\mathbf{A}_{0}}$.

Definition 5.1.1. Let $V$ be a $\mathbb{Q}(p, q)$-vector space and $x \in\{0, \emptyset, \infty\}$. An $\mathbf{A}_{x}$-lattice of $V$ is a free $\mathbf{A}_{x}$-submodule $U_{x}$ of $V$ of $\operatorname{rank} \operatorname{dim}_{\mathbb{Q}(p, q)} V$ such that $\mathbb{Q}(p, q) \otimes_{\mathbf{A}_{x}} U_{x}=$ $V$.

Definition 5.1.2 ([K93, Definition 2.1.2]). Let $V$ be a $\mathbb{Q}(p, q)$-vector space, $U_{x}$ an $\mathbf{A}_{x}$-lattice of $V$ for $x \in\{0, \emptyset, \infty\}$. The triple $\left(U_{0}, U, U_{\infty}\right)$ is said to be balanced if the canonical map

$$
U_{0} \cap U \cap U_{\infty} \rightarrow U_{0} / q U_{0}
$$

is an isomorhism of $\mathbb{Q}$-vector spaces. 
Let $V$ be a $\mathbb{Q}(p, q)$-vector space with a balanced triple $\left(U_{0}, U, U_{\infty}\right)$. Take a $\mathbb{Q}$ basis $\mathcal{B}$ of $U_{0} / q U_{0}$. Since we have an isomorphism $G: U_{0} / q U_{0} \rightarrow U_{0} \cap U \cap U_{\infty}$ of $\mathbb{Q}$-vector spaces, which is the inverse of the canonical map $U_{0} \cap U \cap U_{\infty} \rightarrow U_{0} / q U_{0}$, we obtain an $\mathbf{A}_{x}$-basis $G(\mathcal{B})=\{G(b) \mid b \in \mathcal{B}\}$ of $U_{x}$ for each $x \in\{0, \emptyset, \infty\}$. We call $G(\mathcal{B})$ the global basis of $V$ associated to the balanced triple $\left(U_{0}, U, U_{\infty}\right)$ and the basis $\mathcal{B}$.

Lemma 5.1.3. Let $V, U_{0}, U, U_{\infty}, \mathcal{B}, G$ be as above. Take a subset $\mathcal{B}^{\prime} \subset \mathcal{B}$ and set $U_{x}^{\prime}$ to be the $\mathbf{A}_{x}$-span of $G\left(\mathcal{B}^{\prime}\right):=\left\{G(b) \mid b \in \mathcal{B}^{\prime}\right\}$ for each $x \in\{0, \emptyset, \infty\}$. Also, let $V^{\prime}$ be the $\mathbb{Q}(p, q)$-span of $G\left(\mathcal{B}^{\prime}\right)$. Then, the following hold:

(1) $\left(U_{0}^{\prime}, U^{\prime}, U_{\infty}^{\prime}\right)$ is a balanced triple with the global basis $G\left(\mathcal{B}^{\prime}\right)$.

(2) $\left(U_{0} / U_{0}^{\prime}, U / U^{\prime}, U_{\infty} / U_{\infty}^{\prime}\right)$ is a balanced triple with the global basis $\left\{G(b)+V^{\prime} \mid\right.$ $\left.b \in \mathcal{B} \backslash \mathcal{B}^{\prime}\right\}$.

5.2. Global crystal bases and global y-crystal bases. Let $\mathbf{U}_{\mathbf{A}}$ denote the $\mathbf{A}$ subalgebra of $\mathbf{U}$ generated by $E_{i}^{(n)}, F_{i}^{(n)}, K_{i}^{ \pm 1}, i \in \mathbb{I}, n \in \mathbb{Z}_{>0}$. Similaly, define $\mathbf{U}_{\mathbf{A}}$ to be the A-subalgebra of $\mathbf{U}^{\jmath}$ generated by $e_{i}^{(n)}, f_{i}^{(n)}, k_{i}^{ \pm 1}, i \in \mathbb{I}^{\jmath}, n \in \mathbb{Z}_{>0}$.

Lemma 5.2.1 ([L10, 1.3.5]). Let $A$ be a $\mathbb{Q}(q)$-algebra, $x, y \in A$ such that $x y=$ $q^{2} y x$. Then, for each $n \in \mathbb{Z}_{>0}$, we have

$$
(x+y)^{n}=\sum_{t=0}^{n} q^{t(n-t)}\left[\begin{array}{l}
n \\
t
\end{array}\right] y^{t} x^{n-t} .
$$

Lemma 5.2.2. We have $\mathbf{U}_{\mathbf{A}}^{\jmath} \subset \mathbf{U}_{\mathbf{A}}$.

Proof. It suffices to show that $e_{i}^{(n)}, f_{i}^{(n)} \in \mathbf{U}_{\mathbf{A}}$ for all $i \in \mathbb{I}^{\jmath}, n \in \mathbb{Z}_{>0}$. We prove $e_{i}^{(n)} \in \mathbf{U}_{\mathbf{A}}$; the proof for $f_{i}^{(n)} \in \mathbf{U}_{\mathbf{A}}$ is similar. Setting $x:=E_{\underline{i}}$ and $y:=p^{-\delta_{i, 1}} F_{-\underline{i}} K_{\underline{i}}^{-1}$, we see that

$$
e_{i}=x+y, \quad x y=q^{2} y x .
$$

Then, we can apply Lemma 5.2 .1 , and obtain

$$
e_{i}^{(n)}=\sum_{t=0}^{n} q^{t(n-t)} y^{(t)} x^{(n-t)} .
$$

It is easy to see that $y^{(t)}=p^{-\delta_{i, 1} t} q^{-\delta_{i, 1} \frac{t(t-1)}{2}} F_{-\underline{i}}^{(t)} K_{\underline{i}}^{t} \in \mathbf{U}_{\mathbf{A}}$. Hence, the assertion follows.

Let $V$ be a $\mathbf{U}$-module in $\mathcal{O}_{\text {int }}$ (resp., $\mathbf{U}^{\jmath}$-module in $\mathcal{O}_{\text {int }}^{\jmath}$ ) with a crystal basis $(\mathcal{L}, \mathcal{B})$ (resp., J-crystal basis $(\mathcal{L}, \mathcal{B}))$. Assume that $V$ admits a $\mathbb{Q}$-linear involution - satisfying the following:

$$
\begin{aligned}
\overline{x v} & =\psi(x) \bar{v}, \quad \text { for all } x \in \mathbf{U}, v \in V \\
\text { (resp. }, \overline{x v} & \left.=\psi^{\jmath}(x) \bar{v}, \quad \text { for all } x \in \mathbf{U}^{\jmath}, v \in V\right) .
\end{aligned}
$$

We call such an involution a $\psi$-involution (resp., $\psi^{\jmath}$-involution) on $V$. Since $\mathcal{L}$ is an $\mathbf{A}_{0}$-lattice of $V, \overline{\mathcal{L}}$ is an $\mathbf{A}_{\infty}$-lattice of $V$.

Definition 5.2.3. Let $V, \mathcal{L}, \mathcal{B},-$ be as above. $V$ is said to have a global crystal basis (resp., global j-crystal basis) if there exists a $\mathbf{U}_{\mathbf{A}^{-}}$-submodule (resp., $\mathbf{U}_{\mathbf{A}^{\prime}}$ submodule) $V_{\mathbf{A}}$ of $V$ which is an $\mathbf{A}$-lattice forming a balanced triple $\left(\mathcal{L}, V_{\mathbf{A}}, \overline{\mathcal{L}}\right)$. The associated global basis $G(\mathcal{B})$ (resp., $G^{J}(\mathcal{B})$ ) is called a global crystal basis (resp., global $\boldsymbol{\jmath}$-crystal basis) of $V$. 
Example 5.2.4. Let $\boldsymbol{\lambda} \in P_{1}^{\jmath}$ and consider the irreducible $\mathbf{U}_{1}^{\jmath}$-module $L(\boldsymbol{\lambda})$. Recall that $L(\boldsymbol{\lambda})$ is $\left(\boldsymbol{\lambda}_{0}-\boldsymbol{\lambda}_{-1}+1\right)$-dimensional with a basis $G^{\jmath}(\boldsymbol{\lambda}):=\left\{f_{1}^{(n)} v \mid 0 \leq n \leq\right.$ $\left.\boldsymbol{\lambda}_{0}-\boldsymbol{\lambda}_{-1}\right\}$, where $v$ denotes a highest weight vector. Also, $L(\boldsymbol{\lambda})$ has a $j$-crystal basis $(\mathcal{L}(\boldsymbol{\lambda}), \mathcal{B}(\boldsymbol{\lambda}))$, where $\mathcal{L}(\boldsymbol{\lambda})$ is the $\mathbf{A}_{0}$-span of $G^{\jmath}(\boldsymbol{\lambda})$, and $\mathcal{B}(\boldsymbol{\lambda})=\left\{f_{1}^{(n)} v+q \mathcal{L}(\boldsymbol{\lambda}) \mid\right.$ $\left.0 \leq n \leq \boldsymbol{\lambda}_{0}-\boldsymbol{\lambda}_{-1}\right\}$. Set $L(\boldsymbol{\lambda})_{\mathbf{A}}$ to be the $\mathbf{A}$-span of $G^{\jmath}(\boldsymbol{\lambda})$. Note that there exists a unique $\psi^{\jmath}$-involution $\psi_{\boldsymbol{\lambda}}^{J}$ on $L(\boldsymbol{\lambda})$ fixing $v$. Then, $\left(\mathcal{L}(\boldsymbol{\lambda}), L(\boldsymbol{\lambda})_{\mathbf{A}}, \psi_{\boldsymbol{\lambda}}^{J}(\mathcal{L}(\boldsymbol{\lambda}))\right)$ is a balanced triple, and $G^{\jmath}(\boldsymbol{\lambda})$ is a global $\jmath$-crystal basis of $L(\boldsymbol{\lambda})$.

Proposition 5.2.5. Let $M \in \mathcal{O}_{\text {int }}^{\jmath}$ with a global crystal j-crystal basis $G^{\jmath}\left(\mathcal{B}_{M}\right)$, and $N \in \mathcal{O}_{\text {int }}$ with a global crystal basis $G\left(\mathcal{B}_{N}\right)$ Then, $M \otimes N$ has a global j-crystal basis of the form

$$
\begin{aligned}
& \left\{G^{\jmath}\left(b_{1}\right) \diamond G\left(b_{2}\right) \mid b_{1} \in \mathcal{B}_{M}, b_{2} \in \mathcal{B}_{N}\right\} . \\
& G^{\jmath}\left(b_{1}\right) \diamond G\left(b_{2}\right) \in G^{\jmath}\left(b_{1}\right) \otimes G\left(b_{2}\right) \\
& \quad+\sum_{\substack{b_{1}^{\prime} \in \mathcal{B}_{M}, b_{2}^{\prime} \in \mathcal{B}_{N} \\
\operatorname{wt}\left(b_{2}^{\prime}\right)<\operatorname{wt}\left(b_{2}\right)}} a_{b^{\prime} 1, b_{2}^{\prime} ; b_{1}, b_{2}} G^{\jmath}\left(b_{1}^{\prime}\right) \otimes G\left(b_{2}^{\prime}\right), \quad a_{b^{\prime} 1, b_{2}^{\prime} ; b_{1}, b_{2}} \in \mathbf{A} .
\end{aligned}
$$

Proof. The fact that $\mathcal{B}_{M} \otimes \mathcal{B}_{N}$ forms a -crystal basis of $M \otimes N$ is proved in [W17]. Now, one can construct a global $\jmath$-crystal basis of $M \otimes N$ with the desired property in the same way as the proof of [BWW20, Theorem 4].

5.3. -canonical bases. In this subsection, we recall the notion of $\jmath$-canonical bases, which was introduced by Bao and Wang in BW18a, and explain that ${ }^{-}$ canonical bases are global $\mathrm{f}$-crystal bases. One of the key ingredients for a construction of $\jmath$-canonical bases is the intertwiner $\Upsilon$ :

Definition 5.3.1 ([BW18a, Theorem 6.4]). Let $\mathbf{U}^{-}$denote the subalgebra of $\mathbf{U}$ generated by $F_{i}, i \in \mathbb{I}$. For each $\lambda \in Q_{+}$, there exists a unique $\Upsilon_{\lambda} \in \mathbf{U}_{-\lambda}^{-}$satisfying the following:

- $\Upsilon_{0}=1$,

- $\Upsilon:=\sum_{\lambda \in Q_{+}} \Upsilon_{\lambda}$ satisfies $\psi^{\jmath}(x) \Upsilon=\Upsilon \psi(x)$ for all $x \in \mathbf{U}^{\jmath}$.

Lemma 5.3.2 ([BW18a, Proposition 6.12]). Let $M \in \mathcal{O}_{\text {int }}$ with a $\psi$-involution $\psi_{M}$. Then, the composite $\Upsilon \circ \psi_{M}$ is a $\psi^{\mathrm{J}}$-involution of $M$.

Theorem 5.3.3 (BW18a, Theorem 6.24]). Let $M \in \mathcal{O}_{\text {int }}$ have a global crystal basis $G(\mathcal{B})$ with a crystal basis $(\mathcal{L}, \mathcal{B})$, a $\psi$-involution $\psi_{M}$, and an $\mathbf{A}$-lattice $M_{\mathbf{A}}$. Set $\psi_{M}^{\jmath}:=\Upsilon \circ \psi_{M}$. Then, for each $b \in \mathcal{B}$, there exists a unique $G^{\jmath}(b) \in M$ satisfying the following.

(1) $\psi_{M}^{\jmath}\left(G^{\jmath}(b)\right)=G^{\jmath}(b)$.

(2) $G^{\jmath}(b)=G(b)+\sum_{b^{\prime} \in B} c_{b^{\prime}, b} G\left(b^{\prime}\right)$ for some $c_{b^{\prime}, b} \in q \mathbf{A}_{0} \cap \mathbf{A}$. Moreover, $c_{b^{\prime}, b}=0$ unless $\mathrm{wt}^{\jmath}\left(b^{\prime}\right)=\mathrm{wt}^{\jmath}(b)$ and $\operatorname{wt}\left(b^{\prime}\right)<\mathrm{wt}(b)$.

The new basis $G^{\jmath}(\mathcal{B}):=\left\{G^{\jmath}(b) \mid b \in \mathcal{B}\right\}$ thus constructed is called the $\mathrm{\jmath}$-canonical basis of $(M, G(\mathcal{B}))$.

Proposition 5.3.4. We keep the notation in Theorem 5.3 .3 . Then, $(\mathcal{L}, \mathcal{B})$ is a $\mathrm{J}$ crystal basis, $\left(\mathcal{L}, M_{\mathbf{A}}, \psi_{M}^{\jmath}(\mathcal{L})\right)$ is a balanced triple, and $G^{\jmath}(\mathcal{B})$ is the global j-crystal basis associated to the balanced triple $\left(\mathcal{L}, M_{\mathbf{A}}, \psi_{M}^{J}(\mathcal{L})\right)$ and the basis $\mathcal{B}$. 
Proof. That $(\mathcal{L}, \mathcal{B})$ is a $\jmath$-crystal basis has already been stated in Theorem 4.2.2. Let us prove the rest. By the property $(2)$ of Theorem 5.3.3, it is clear that $\mathcal{L}$ (resp., $M_{\mathbf{A}}$ ) is spanned by $G^{\jmath}(\mathcal{B})$ over $\mathbf{A}_{0}$ (resp., A). Also, by (1) of Theorem 5.3.3. $\psi_{M}^{J}(\mathcal{L})$ is spanned by $G^{\jmath}(\mathcal{B})$ over $\mathbf{A}_{\infty}$. Hence, the canonical homomorphism $\mathcal{L} \cap M_{\mathbf{A}} \cap \psi_{M}^{3}(\mathcal{L}) \rightarrow \mathcal{L} / q \mathcal{L}$ is an isomorphism, and therefore, $\left(\mathcal{L}, M_{\mathbf{A}}, \psi_{M}^{3}(\mathcal{L})\right)$ is balanced. Finally, by Lemma 5.2.2 the $\mathbf{U}_{\mathbf{A}}$-module $M_{\mathbf{A}}$ is also a $\mathbf{U}_{\mathbf{A}}^{3}$-module. This proves the proposition.

\section{KAZHDAN-Lusztig BASES}

The subsequent three sections are dedicated to prove the existence of a global J-crystal basis and its "dual" basis for $L(\boldsymbol{\lambda}), \boldsymbol{\lambda} \in P^{\jmath}$. In this section, we formulate variants of the Kazhdan-Lusztig bases following [KL79], Deo87], and [L03.

6.1. Hecke algebra of type $B$. Fix $d \in \mathbb{Z}_{>0}$. Let $W=W_{d}$ be the Weyl group of type $B_{d}$ with simple reflections $S=\left\{s_{0}, s_{1}, \ldots, s_{d-1}\right\}$ such that

$$
s_{0} s_{1} s_{0} s_{1}=s_{1} s_{0} s_{1} s_{0}, \quad s_{i} s_{i+1} s_{i}=s_{i+1} s_{i} s_{i+1} \text { if } i \geq 1, \quad s_{i} s_{j}=s_{j} s_{i} \text { if }|i-j|>1 .
$$

Definition 6.1.1. The Hecke algebra $\mathcal{H}=\mathcal{H}(W)$ associated to $W$ with unequal parameters $p, q$ is the associative algebra over $\mathbf{A}_{\mathbb{Z}}:=\mathbb{Z}\left[p, p^{-1}, q, q^{-1}\right]$ generated by $\left\{H_{s} \mid s \in S\right\}$ subject to the following relations:

- $\left(H_{s}-q_{s}^{-1}\right)\left(H_{s}+q_{s}\right)=0$ for all $s \in S$, where $q_{s}=p$ if $s=s_{0}$ and $q_{s}=q$ otherwise.

- $H_{s_{0}} H_{s_{1}} H_{s_{0}} H_{s_{1}}=H_{s_{1}} H_{s_{0}} H_{s_{1}} H_{s_{0}}$.

- $H_{s_{i}} H_{s_{i+1}} H_{s_{i}}=H_{s_{i+1}} H_{s_{i}} H_{s_{i+1}}$ if $i \geq 1$.

- $H_{s_{i}} H_{s_{j}}=H_{s_{j}} H_{s_{i}}$ if $|i-j|>1$.

We often write $H_{i}=H_{s_{i}}$. For each $w \in W$ with a reduced expression $w=$ $s_{i_{1}} \cdots s_{i_{l}}$, the product $H_{i_{1}} \cdots H_{i_{l}}$ is independent of the choice of a reduced expression of $w$; we denote it by $H_{w}$. Similarly, $q_{w}:=q_{s_{i_{1}}} \cdots q_{s_{i_{l}}}$ is well-defined.

Let $U, V$ be modules over $\mathbf{A}_{\mathbb{Z}}$. We say a $\mathbb{Z}$-linear map $f: U \rightarrow V$ is anti-linear if it satisfies $f(g u)=\bar{g} f(u)$ for all $g \in \mathbf{A}_{\mathbb{Z}}$ and $u \in U$. In the sequel, we will often use the following automorphisms, all of which are involutions, of $\mathcal{H}$.

\section{Lemma 6.1.2.}

(1) There exists a unique anti-linear algebra automorphism $\div$ of $\mathcal{H}$ such that $\overline{H_{w}}=H_{w^{-1}}^{-1}$.

(2) There exists a unique anti-linear algebra automorphism sgn of $\mathcal{H}$ such that $\operatorname{sgn}\left(H_{w}\right)=(-1)^{\ell(w)} H_{w}$. Here, $\ell: W \rightarrow \mathbb{Z}_{\geq 0}$ denotes the length function on $W$.

(3) There exists a unique $\mathbf{A}_{\mathbb{Z}}$-algebra anti-automorphism $(\cdot)^{b}$ of $\mathcal{H}$ such that $H_{w}^{b}=H_{w^{-1}}$.

Moreover, all of these automorphisms commute with each other.

For $y, w \in W$, define $r_{y, w} \in \mathbf{A}_{\mathbb{Z}}$ by

$$
\overline{H_{w}}=\sum_{y \in W} r_{y, w} H_{y} .
$$

It is well-known and easily proved that $r_{w, w}=1$ for all $w \in W$ and $r_{y, w}=0$ unless $y \leq w$. 
6.2. Kazhdan-Lusztig bases. Let us formulate the Kazhdan-Lusztig basis and the dual Kazhdan-Lusztig basis. Set

$$
\begin{aligned}
& \mathbf{A}_{\mathbb{Z}}^{+}:=\mathbf{A}_{\mathbb{Z}} \cap \mathbf{A}_{0}=p \mathbb{Z}\left[p, q, q^{-1}\right] \oplus q \mathbb{Z}[q], \\
& \mathbf{A}_{\mathbb{Z}}^{-}:=\overline{\mathbf{A}_{\mathbb{Z}}^{+}}=p^{-1} \mathbb{Z}\left[p^{-1}, q, q^{-1}\right] \oplus q^{-1} \mathbb{Z}\left[q^{-1}\right] .
\end{aligned}
$$

Theorem 6.2.1 ([KL79, Theorem 1.1], [L03, Theorem 5.2]). For each $w \in W$, there exists a unique $C_{w} \in \mathcal{H}$ such that

(1) $\overline{C_{w}}=C_{w}$.

(2) $C_{w}=H_{w}+\sum_{y<w} c_{y, w} H_{y}$ for some $c_{y, w} \in \mathbf{A}_{\mathbb{Z}}^{+}$. Here, $<$denotes the Bruhat order on $W$.

Replacing $\mathbf{A}_{\mathbb{Z}}^{+}$with $\mathbf{A}_{\mathbb{Z}}^{-}$, we see the following: For each $w \in W$, there exists a unique $D_{w} \in \mathcal{H}$ such that

(1) $\overline{D_{w}}=D_{w}$.

(2) $D_{w}=H_{w}+\sum_{y<w} d_{y, w} H_{y}$ for some $d_{y, w} \in \mathbf{A}_{\mathbb{Z}}^{-}$.

Remark 6.2.2. Noting that the automorphisms - and $\operatorname{sgn}$ commute with each other, it is easy to verify that $D_{w}=(-1)^{\ell(w)} \operatorname{sgn}\left(C_{w}\right)$.

It is obvious from the definitions that both $\left\{C_{w} \mid w \in W\right\}$ and $\left\{D_{w} \mid w \in W\right\}$ form $\mathbf{A}_{\mathbb{Z}}$-bases of $\mathcal{H}$. We call the former the Kazhdan-Lusztig basis, and the latter the dual Kazhdan-Lusztig basis of $\mathcal{H}$.

6.3. Left cell representations. Let us recall from [KL79] the notion of left cells of $W$ and the associated left cell representations.

Definition 6.3.1. Let $y, w \in W$.

(1) $y \rightarrow_{L} w$ if the coefficient of $C_{y}$ in $C_{s} C_{w}$ expanded in the Kazhdan-Lusztig basis is nonzero for some $s \in S$.

(2) $y \leq_{L} w$ if there exist $y=y_{0}, y_{1}, \ldots, y_{l}=w \in W$ such that $y_{i-1} \rightarrow_{L} y_{i}$.

(3) $y \sim_{L} w$ if $y \leq_{L} w$ and $w \leq_{L} y$.

(4) $y<_{L} w$ if $y \leq_{L} w$ and $y \neq_{L} w$.

(5) Each equivalence class of $W / \underset{L}{\sim}$ is called a left cell of $W$. We denote by $L(W)$ the set of left cells of $W$.

Remark 6.3.2. By Remark 6.2 .2 , we obtain the same equivalence relation as $\underset{L}{\sim}$ if we replace $C_{w}$ 's by $D_{w}$ 's.

For each $X \in L(W)$ and $x \in X$, set

$$
\begin{aligned}
& C_{\leq_{L} X}=\bigoplus_{y \leq_{L} x} \mathbf{A}_{\mathbb{Z}} C_{y}, \quad C_{<_{L} X}=\bigoplus_{y<L_{L} x} \mathbf{A}_{\mathbb{Z}} C_{y}, \quad C_{X}^{L}=C_{\leq_{L} X} / C_{<_{L} X}, \\
& D_{\leq_{L} X}=\bigoplus_{y \leq_{L} x} \mathbf{A}_{\mathbb{Z}} D_{y}, \quad D_{<_{L} X}=\bigoplus_{y<_{L} x} \mathbf{A}_{\mathbb{Z}} D_{y}, \quad D_{X}^{L}=D_{\leq_{L} X} / D_{<_{L} X} .
\end{aligned}
$$

Note that these are independent of the choice of $x \in X$. We denote the image of $m \in C_{\leq_{L} X}$ (resp., $m \in D_{\leq_{L} X}$ ) under the quotient map $C_{\leq_{L} X} \rightarrow C_{X}^{L}$ (resp., $\left.D_{\leq_{L} X} \rightarrow D_{X}^{L}\right)$ by $[m]_{X}$ (resp., $\left.[m]_{X}^{\prime}\right)$.

Lemma 6.3.3. Let $X \in L(W)$. Then, $C_{\leq_{L} X}, C_{<_{L} X}, D_{\leq_{L} X}$, and $D_{<_{L} X}$ are left ideals of $\mathcal{H}$, and consequently, $C_{X}^{L}$ and $D_{X}^{L}$ are left $\mathcal{H}$-modules. Moreover, $C_{X}^{L}$ has a basis $\left\{\left[C_{x}\right]_{X} \mid x \in X\right\}$, while $D_{X}^{L}$ has a basis $\left\{\left[D_{x}\right]_{X}^{\prime} \mid x \in X\right\}$. 
Proof. The assertions are obvious from the definitions.

We call $C_{X}^{L}$ the left cell representation of $\mathcal{H}(W)$ associated to $X \in L(W)$.

6.4. Bilinear form on $\mathcal{H}$. Let $\mathcal{H}^{*}:=\operatorname{Hom}_{\mathbf{A}_{\mathbb{Z}}}\left(\mathcal{H}, \mathbf{A}_{\mathbb{Z}}\right)$. $\mathcal{H}^{*}$ has a left $\mathcal{H}$-module structure given by

$$
(H f)\left(H^{\prime}\right)=f\left(H^{b} H^{\prime}\right), \quad \text { for all } f \in \mathcal{H}^{*}, H, H^{\prime} \in \mathcal{H} .
$$

Let $\left\{h_{w} \mid w \in W\right\} \subset \mathcal{H}^{*}$ be the dual basis of $\left\{H_{w} \mid w \in W\right\}$, that is, they are characterized by $h_{y}\left(H_{w}\right)=\delta_{y, w}$ for all $y, w \in W$.

Lemma 6.4.1. For each $w \in W$ and $s \in S$, the following holds.

$$
H_{s} h_{w}= \begin{cases}h_{s w} & \text { if } w<s w, \\ h_{s w}+\left(q_{s}^{-1}-q_{s}\right) h_{w} & \text { if } s w<w .\end{cases}
$$

Proof. For each $y \in W$, we compute as

$$
\begin{aligned}
\left(H_{s} h_{w}\right)\left(H_{y}\right) & =h_{w}\left(H_{s} H_{y}\right) \\
& = \begin{cases}h_{w}\left(H_{s y}\right) & \text { if } s y>y, \\
h_{w}\left(H_{s y}+\left(q_{s}^{-1}-q_{s}\right) H_{y}\right) & \text { if } s y<y\end{cases} \\
& = \begin{cases}1 & \text { if } s y>y \text { and } s y=w, \\
1 & \text { if } s y<y \text { and } s y=w, \\
q_{s}^{-1}-q_{s} & \text { if } s y<y \text { and } y=w, \\
0 & \text { otherwise }\end{cases} \\
& = \begin{cases}h_{s w}\left(H_{y}\right) & \text { if } s w>w, \\
\left(h_{s w}+\left(q_{s}^{-1}-q_{s}\right) h_{w}\right)\left(H_{y}\right) & \text { if } s w<w .\end{cases}
\end{aligned}
$$

This implies

$$
H_{s} h_{w}= \begin{cases}h_{s w} & \text { if } s w>w, \\ h_{s w}+\left(q_{s}^{-1}-q_{s}\right) h_{w} & \text { if } s w<w .\end{cases}
$$

Thus, the proof completes.

There exists an anti-linear automorphism $\div$ of $\mathcal{H}^{*}$ defined by $\bar{f}(H)=\overline{f(\bar{H})}$ for $f \in \mathcal{H}^{*}, H \in \mathcal{H}$.

Lemma 6.4.2. For each $w \in W$, we have

$$
\overline{h_{w}}=\sum_{y \geq w} \overline{r_{w, y}} h_{y} .
$$

In particular, $\overline{h_{w_{0}}}=h_{w_{0}}$, where $w_{0} \in W$ denotes the longest element.

Proof. Let $y \in W$. Then, we have

$$
\overline{h_{w}}\left(H_{y}\right)=\overline{h_{w}\left(\overline{H_{y}}\right)}=\overline{h_{w}\left(\sum_{z \leq y} r_{z, y} H_{z}\right)}=\overline{r_{w, y}} .
$$

Since $\overline{h_{w}}=\sum_{y \in W} \overline{h_{w}}\left(H_{y}\right) h_{y}$, the assertion follows.

Let $\left\{C_{w}^{*} \mid w \in W\right\} \subset \mathcal{H}^{*}$ denote the dual basis of $\left\{C_{w} \mid w \in W\right\}$. 
Proposition 6.4.3. $C_{w}^{*}$ is characterized by the following two conditions:

(1) $\overline{C_{w}^{*}}=C_{w}^{*}$.

(2) $C_{w}^{*}=h_{w}+\sum_{z>w} c_{w, z}^{*} h_{z}$ for some $c_{w, z}^{*} \in \mathbf{A}_{\mathbb{Z}}^{+}$.

Proof. Thanks to Lemma 6.4.2, one can prove that there exists a unique $C_{w}^{\prime} \in \mathcal{H}^{*}$ such that $\overline{C_{w}^{\prime}}=C_{w}^{\prime}$ and $C_{w}^{\prime}-h_{w} \in \bigoplus_{y>w} \mathbf{A}_{\mathbb{Z}}^{+} h_{y}$ in a similar way to Theorem 6.2.1. Hence, it suffices to show that $C_{w}^{*}$ satisfies the two conditions.

The first condition is verified as follows. For each $y \in W$, we have

$$
\overline{C_{w}^{*}}\left(C_{y}\right)=\overline{C_{w}^{*}\left(\overline{\left.C_{y}\right)}\right.}=\overline{C_{w}^{*}\left(C_{y}\right)}=\overline{\delta_{y, w}}=\delta_{y, w}=C_{w}^{*}\left(C_{y}\right) .
$$

Since $\left\{C_{y} \mid y \in W\right\}$ is a basis of $\mathcal{H}$, we obtain $\overline{C_{w}^{*}}=C_{w}^{*}$.

Next, we prove the second condition. For each $y \in W$, we can write $H_{y}=$ $C_{y}+\sum_{z<y} b_{z, y} C_{z}$ for some $b_{z, y} \in \mathbf{A}_{\mathbb{Z}}^{+}$. Then, we have

$$
C_{w}^{*}=\sum_{y \in W} C_{w}^{*}\left(H_{y}\right) h_{y}=h_{w}+\sum_{y>w} b_{w, y} h_{y} .
$$

This completes the proof.

Lemma 6.4.4. The linear map $d: \mathcal{H} \rightarrow \mathcal{H}^{*} ; H \mapsto H \cdot h_{w_{0}}$ gives an isomorphism of left $\mathcal{H}$-modules. Moreover, we have

(1) $d\left(\overline{H_{y}}\right)=h_{y w_{0}}$ for all $y \in W$.

(2) $d(\bar{H})=\overline{d(H)}$ for all $H \in \mathcal{H}$.

Proof. By Lemma 6.4.1, the linear map $\varphi: \mathcal{H} \rightarrow \mathcal{H}^{*} ; H_{w} \mapsto h_{w}$ is an isomorphism of left $\mathcal{H}$-modules. On the other hand, the map $\psi: \mathcal{H} \rightarrow \mathcal{H} ; H \mapsto H \cdot H_{w_{0}}$ is clearly an isomorphism of left $\mathcal{H}$-modules. Thus, the composite map $d:=\varphi \circ \psi: \mathcal{H} \rightarrow \mathcal{H}^{*}$ is an isomorphism of left $\mathcal{H}$-modules satisfying

$$
d(H)=\varphi\left(H \cdot H_{w_{0}}\right)=H \cdot \varphi\left(H_{w_{0}}\right)=H \cdot h_{w_{0}} \quad \text { for all } H \in \mathcal{H} .
$$

Also, we have, for all $y \in W$,

$$
d\left(\overline{H_{y}}\right)=\varphi\left(\overline{H_{y}} \cdot H_{w_{0}}\right)=\varphi\left(H_{y^{-1}}^{-1} \cdot H_{y^{-1}} H_{y w_{0}}\right)=\varphi\left(H_{y w_{0}}\right)=h_{y w_{0}} .
$$

Finally, for each $H, H^{\prime} \in \mathcal{H}$, we have

$$
\begin{aligned}
& d(\bar{H})\left(H^{\prime}\right)=\left(\bar{H} \cdot h_{w_{0}}\right)\left(H^{\prime}\right)=h_{w_{0}}\left((\bar{H})^{b} H^{\prime}\right), \\
& \overline{d(H)}\left(H^{\prime}\right)=\overline{d(H)\left(\overline{H^{\prime}}\right)}=\overline{h_{w_{0}}\left(H^{b} \overline{H^{\prime}}\right)}=\overline{h_{w_{0}}}\left(\overline{H^{b}} H^{\prime}\right) .
\end{aligned}
$$

Then, the equality $d(\bar{H})=\overline{d(H)}$ follows from the facts that $\overline{h_{w_{0}}}=h_{w_{0}}$ and $(\bar{H})^{b}=$ $\overline{H^{b}}$; the former is proved in Lemma 6.4.2, and the latter is in Lemma 6.1.2.

Using this isomorphism, we define a bilinear form $\langle\cdot \mid \cdot\rangle$ on $\mathcal{H}$ by

$$
\left\langle H \mid H^{\prime}\right\rangle:=d\left(H^{\prime}\right)(H), \quad\left(H, H^{\prime} \in \mathcal{H}\right) .
$$

Clearly, this bilinear form satisfies $\left\langle H^{\prime} \mid H H^{\prime \prime}\right\rangle=\left\langle H^{b} H^{\prime} \mid H^{\prime \prime}\right\rangle$ for all $H, H^{\prime}, H^{\prime \prime} \in$ $\mathcal{H}$.

Lemma 6.4.5. The bilinear from $\langle\cdot \mid \cdot\rangle$ is symmetric.

Proof. Let $H_{1}, H_{2} \in \mathcal{H}$. It suffices to show that $h_{w_{0}}\left(H_{2}^{b} H_{1}\right)=h_{w_{0}}\left(H_{1}^{b} H_{2}\right)$. Since $H_{w_{0}}^{b}=H_{w_{0}}$, it holds that $h_{w_{0}}\left(H^{b}\right)=h_{w_{0}}(H)$ for all $H \in \mathcal{H}$. Then, the assertion follows if one notes $\left(H_{2}^{b} H_{1}\right)^{b}=H_{1}^{b} H_{2}$. 
Proposition 6.4.6. The bases $\left\{C_{w} \mid w \in W\right\}$ and $\left\{D_{w w_{0}} \mid w \in W\right\}$ are dual to each other with respect to $\langle\cdot \mid \cdot\rangle$, that is, we have $\left\langle C_{y} \mid D_{w}\right\rangle=\delta_{y, w w_{0}}$ for all $y, w \in W$.

Proof. Recall that $D_{w}=\sum_{y \leq w} d_{y, w} H_{y}$ with $d_{w, w}=1$ and $d_{y, w} \in \mathbf{A}_{\mathbb{Z}}^{-}$for all $y<w$. Then, we have

$$
\begin{aligned}
& \overline{d\left(D_{w}\right)}=d\left(\overline{D_{w}}\right)=d\left(D_{w}\right), \\
& d\left(D_{w}\right)=d\left(\overline{D_{w}}\right)=d\left(\sum_{y \leq w} \overline{d_{y, w} H_{y}}\right)=\sum_{y \leq w} \overline{d_{y, w}} h_{y w_{0}}=\sum_{z \geq w w_{0}} \overline{d_{z w_{0}, w}} h_{z} .
\end{aligned}
$$

This and Proposition 6.4.3 show that $d\left(D_{w}\right)=C_{w w_{0}}^{*}$. Hence, it holds that $\left\langle C_{y}\right.$ | $\left.D_{w}\right\rangle=C_{w w_{0}}^{*}\left(C_{y}\right)=\delta_{y, w w_{0}}$, which proves the proposition.

Here, we describe the duality between $C_{X}^{L}$ 's and $D_{X}^{L}$ 's.

Lemma 6.4.7. Let $y, w \in W, X \in L(W)$. Then, the following hold.

(1) $y \rightarrow_{L} w$ if and only if $w w_{0} \rightarrow_{L} y w_{0}$.

(2) $y \leq_{L} w$ if and only if $w w_{0} \leq_{L} y w_{0}$.

(3) $X w_{0}:=\left\{x w_{0} \mid x \in X\right\} \in L(W)$.

Proof. We first prove part (1). Suppose that $y \rightarrow_{L} w$. Then, there exists $s \in S$ such that $\left\langle C_{s} C_{w} \mid D_{y w_{0}}\right\rangle \neq 0$. This implies that $\left\langle C_{w} \mid C_{s} D_{y w_{0}}\right\rangle \neq 0$, and hence, we obtain $w w_{0} \rightarrow_{L} y w_{0}$. Replacing $y, w$ by $y w_{0}, w w_{0}$, we also have the opposite indication. This proves part (1). Assertion (2) is an immediate consequence of (1). We prove part (3). Let $x \in X$. Then, $X=\left\{y \in W \mid x \leq_{L} y \leq_{L} x\right\}$. By part (2), we have $x \leq_{L} y \leq_{L} x$ if and only if $x w_{0} \leq_{L} y w_{0} \leq_{L} x w_{0}$. This implies that $X w_{0}=\left\{z \in W \mid x w_{0} \leq_{L} z \leq_{L} x w_{0}\right\}$, and it is a unique left cell of $W$ containing $x w_{0}$. Thus, the proof completes.

Lemma 6.4.8. The bilinear from $\langle\cdot \mid \cdot\rangle$ induces a non-degenerate bilinear form on $C_{X}^{L} \times D_{X w_{0}}^{L}$. Moreover, $\left\{\left[C_{x}\right]_{X} \mid x \in X\right\}$ and $\left\{\left[D_{x w_{0}}\right]_{X w_{0}}^{\prime} \mid x \in X\right\}$ form bases which are dual to each other.

Proof. Let $x \in X, y, w \in W$ be such that $y<_{L} x$ and $w w_{0}<_{L} x w_{0}$. It suffices to show that $\left\langle C_{y} \mid D_{u}\right\rangle=0$ for all $u \leq_{L} x w_{0}$ and $\left\langle C_{v} \mid D_{w w_{0}}\right\rangle=0$ for all $v \leq_{L} x$. Both are obvious from Lemma 6.4.7 (2).

Proposition 6.4.9. Let $X \in L(W)$. Then, we have an isomorphism $D_{X w_{0}}^{L} \simeq C_{X}^{L}$ of $\mathcal{H}$-modules.

Proof. It suffices to show that the characters $\operatorname{ch}_{D_{X w_{0}}^{L}}$ of $D_{X w_{0}}^{L}$ and $\operatorname{ch}_{C_{X}^{L}}$ of $C_{X}^{L}$ coincide with each other. For each $w \in W$, we compute as

$$
\begin{aligned}
\operatorname{ch}_{C_{X}^{L}}\left(H_{w}\right) & =\sum_{x \in X}\left\langle H_{w}\left[C_{x}\right]_{X} \mid\left[D_{x w_{0}}\right]_{X w_{0}}^{\prime}\right\rangle \\
& =\sum_{x \in X}\left\langle\left[C_{x}\right]_{X} \mid H_{w^{-1}}\left[D_{x w_{0}}\right]_{X w_{0}}^{\prime}\right\rangle \\
& =\operatorname{ch}_{D_{X w_{0}}^{L}}\left(H_{w^{-1}}\right)=\operatorname{ch}_{D_{X w_{0}}^{L}}\left(H_{w}\right) .
\end{aligned}
$$

Thus, the proof completes. 
6.5. Parabolic Kazhdan-Lusztig bases. Throughout this subsection, we fix a subset $J \subset\{0,1, \ldots, d-1\}$ arbitrarily. Let $W_{J}$ denote the parabolic subgroup of $W$ generated by $\left\{s_{j} \mid j \in J\right\},{ }^{J} W$ the set of minimal length coset representatives for $W_{J} \backslash W$, and $w_{J} \in W_{J}$ the longest element. Also, we set

$$
x_{J}:=q_{w_{J}} \sum_{w \in W_{J}} q_{w}^{-1} H_{w} \in \mathcal{H}
$$

Lemma 6.5.1. Let $j \in J$. Then, the following hold.

(1) $x_{J} H_{j}=q_{s_{j}}^{-1} x_{J}$.

(2) $x_{J}^{b}=x_{J}$.

(3) $x_{J}=C_{w_{J}}$. In particular, $\overline{x_{J}}=x_{J}$.

Proof. The assertion (1) follows from a direct calculation and the fact that $W_{J}=$ $\left\{w \in W_{J} \mid w<s_{j} w\right\} \sqcup\left\{w \in W_{J} \mid s_{j} w<w\right\}$. The assertion (2) follows from the definition of $x_{J}$ and the facts that $W_{J}=\left\{w^{-1} \mid w \in W_{J}\right\}$, and $q_{w^{-1}}=q_{w}$ for all $w \in W$. The proof of (3) can be found in [X94, Proposition 1.17 (2)].

By Lemma 6.5.1 (1), the right ideal $x_{J} \mathcal{H}$ of $\mathcal{H}$ has a basis $\left\{x_{J} H_{w} \mid w \in{ }^{J} W\right\}$. Also, by Lemma 6.5.1 (3), $x_{J} \mathcal{H}$ is closed under the involution - . Hence, we can construct analogs of the Kazhdan-Lusztig basis and the dual Kazhdan-Lusztig basis of $\mathcal{H}$ in the ideal $x_{J} \mathcal{H}$ :

Theorem 6.5.2 ([Deo87, Proposition 3.2]).

(1) For each $w \in{ }^{J} W$, there exists a unique ${ }^{J} C_{w} \in x_{J} \mathcal{H}$ such that

(a) $\overline{{ }^{J} C_{w}}={ }^{J} C_{w}$.

(b) ${ }^{J} C_{w}=x_{J}\left(H_{w}+\sum_{\substack{y \in \in^{J} W \\ y<w}}{ }^{J} c_{y, w} H_{y}\right)$ for some ${ }^{J} c_{y, w} \in \mathbf{A}_{\mathbb{Z}}^{+}$.

(2) For each $w \in{ }^{J} W$, there exists a unique ${ }^{J} D_{w} \in x_{J} \mathcal{H}$ such that

(a) $\overline{{ }^{J} D_{w}}={ }^{J} D_{w}$.

(b) ${ }^{J} D_{w}=x_{J}\left(H_{w}+\sum_{\substack{y \in \in^{J} W \\ y<w}}^{J} d_{y, w} H_{y}\right)$ for some ${ }^{J} d_{y, w} \in \mathbf{A}_{\mathbb{Z}}^{-}$.

Clearly, $\left\{{ }^{J} C_{w} \mid w \in{ }^{J} W\right\}$ and $\left\{{ }^{J} D_{w} \mid w \in{ }^{J} W\right\}$ are linear bases of $x_{J} \mathcal{H}$. We call them the parabolic Kazhdan-Lusztig basis and the dual parabolic Kazhdan-Lusztig basis of $x_{J} \mathcal{H}$, respectively.

Proposition 6.5.3 ([Deo87, Proposition 3.4]). Let $w \in{ }^{J} W$. Then, ${ }^{J} C_{w}=C_{w_{J} w}$.

Proposition 6.5.4. Let $w \in{ }^{J} W$. Then, ${ }^{J} D_{w}=x_{J} D_{w}$. 
Proof. For each $y \in W$, define $y_{J} \in W_{J}$ and ${ }^{J} y \in{ }^{J} W$ to be the unique elements satisfying $y=y_{J}{ }^{J} y$ and $\ell(y)=\ell\left(y_{J}\right)+\ell\left({ }^{J} y\right)$. Then, we have

$$
\begin{aligned}
x_{J} D_{w} & =x_{J} \sum_{y \leq w} d_{y, w} H_{y} \\
& =x_{J}\left(H_{w}+\sum_{y<w} d_{y, w} H_{y_{J}} H_{J}\right) \\
& =x_{J}\left(H_{w}+\sum_{y<w} q_{y_{J}}^{-1} d_{y, w} H_{J}\right) \quad \text { (by Lemma 6.5.1 (1)) } \\
& =x_{J}\left(H_{w}+\sum_{\substack{y \in \in^{J} W \\
y<w}} \sum_{\substack{x \in W_{J} \\
x y<w}} q_{x}^{-1} d_{x y, w} H_{y}\right) .
\end{aligned}
$$

This shows that $x_{J} D_{w}-x_{J} H_{w} \in \bigoplus_{\substack{y \in \in^{J} W \\ y<w}} \mathbf{A}_{\mathbb{Z}}^{-} x_{J} H_{y}$. Hence, by Theorem 6.5.2 (2), $x_{J} D_{w}$ coincides with ${ }^{J} D_{w}$.

For a later use, let us consider $x_{J} C_{y}$ and $x_{J} D_{y}$ for $y \in W$.

Proposition 6.5.5. Let $y \in W$. Then, we have

$$
x_{J} C_{y}=\sum_{\substack{w \in \in^{J} W \\ w_{J} w \leq L}} \alpha_{w}{ }^{J} C_{w},
$$

for some $\alpha_{w} \in \mathbf{A}_{\mathbb{Z}}$.

Proof. Let us write

$$
x_{J} C_{y}=\sum_{w \in{ }^{J} W} \alpha_{w}{ }^{J} C_{w}=\sum_{w \in{ }^{J} W} \alpha_{w} C_{w_{J} w} \quad \text { for some } \alpha_{w} \in \mathbf{A}_{\mathbb{Z}} .
$$

Also, by the definition of $\leq_{L}$, we can write

$$
x_{J} C_{y}=\sum_{z \leq L} \beta_{z} C_{z} \quad \text { for some } \beta_{z} \in \mathbf{A}_{\mathbb{Z}} .
$$

This shows $\alpha_{w}=0$ unless $w_{J} w \leq_{L} y$.

Lemma 6.5.6 ([L03, Theorem 6.6 (b)]). Let $w \in W$ and $s \in S$ be such that $s w<w$. Then, it holds that $H_{s} D_{w}=-q_{s} D_{w}$.

Proposition 6.5.7. Let $y \in W \backslash{ }^{J} W$. Then, $x_{J} D_{y}=0$.

Proof. Since $y \notin{ }^{J} W$, there exists $j \in J$ such that $s_{j} y<y$. For such $j$, we have $x_{J} H_{j}=q_{j}^{-1} x_{J}$ (Lemma 6.5.1 (1)) and $H_{j} D_{y}=-q_{j} D_{y}$ (Lemma 6.5.6). Hence, we obtain

$$
x_{J} D_{y}=q_{j} x_{J} H_{j} D_{y}=-q_{j}^{2} x_{J} D,
$$

which implies $x_{J} D_{y}=0$, as desired.

Set $P_{J}:=q_{w_{J}} \sum_{x \in W_{J}} q_{x}^{-2} \in \mathbf{A}_{\mathbb{Z}}$. Note that, by Lemma 6.5.1 (1), it holds that $x_{J}^{2}=P_{J} x_{J}$. Then, for each $H, H^{\prime} \in \mathcal{H}$, we have

$$
\left\langle x_{J} H \mid x_{J} H^{\prime}\right\rangle=\left\langle x_{J}^{2} H \mid H^{\prime}\right\rangle=P_{J}\left\langle H \mid H^{\prime}\right\rangle \in P_{J} \mathbf{A}_{\mathbb{Z}} ;
$$


here, we use Lemma 6.5.1 (2). Hence, we can define an $\mathbf{A}_{\mathbb{Z}}$-valued bilinear form $\langle\cdot \mid \cdot\rangle_{J}$ on $x_{J} \mathcal{H}$ by $\langle\cdot \mid \cdot\rangle_{J}:=\frac{1}{P_{J}}\langle\cdot \mid \cdot\rangle$.

Proposition 6.5.8. The basis $\left\{{ }^{J} C_{w} \mid w \in{ }^{J} W\right\}$ and $\left\{{ }^{J} D_{w_{J} w w_{0}} \mid w \in{ }^{J} W\right\}$ are dual to each other with respect to $\langle\cdot \mid \cdot\rangle_{J}$, that is, we have $\left\langle{ }^{J} C_{y} \mid{ }^{J} D_{w}\right\rangle_{J}=\delta_{y, w_{J} w w_{0}}$ for all $y, w \in{ }^{J} W$.

Proof. Let $y, w \in{ }^{J} W$. We compute as follows:

$$
\begin{aligned}
\left\langle{ }^{J} C_{y} \mid{ }^{J} D_{w}\right\rangle_{J} & =\frac{1}{P_{J}}\left\langle{ }^{J} C_{y} \mid{ }^{J} D_{w}\right\rangle \\
& =\frac{1}{P_{J}}\left\langle C_{w_{J} y} \mid x_{J} D_{w}\right\rangle \quad \text { (by Proposition 6.5.3 and 6.5.4) } \\
& =\left\langle C_{w_{J} y} \mid D_{w}\right\rangle \quad\left(\text { since } C_{w_{J} y}={ }^{J} C_{y} \in x_{J} \mathcal{H}\right) \\
& =\delta_{w_{J} y, w w_{0}}=\delta_{y, w_{J} w w_{0}} \quad \text { (by Proposition 6.4.6). }
\end{aligned}
$$

This proves the proposition.

\section{HeCKe MOdules AND THEIR CENTRALIZERS}

In this section, we study the centralizer algebras of certain modules over the Hecke algebra. They are known as (generalized) $q$-Schur algebras. Multiparameter $q$-Schur algebra of type B is also studied in [LL19].

7.1. Fundamental properties. We follow ideas in DDPW08, Chapter 9.1]. Let $\pi$ be an index set. Suppose that we are given a map $\pi \rightarrow\{J \mid J \subset\{0,1, \ldots, d-1\}\}$. We denote by $I_{\lambda}$ the image of $\lambda \in \pi$ under this map. For each $\lambda \in \pi$, for simplicity, we will denote $W_{I_{\lambda}}, w_{I_{\lambda}}, x_{I_{\lambda}}$, etc. by $W_{\lambda}, w_{\lambda}, x_{\lambda}$, etc.

Definition 7.1.1. Associated with $\pi$, we define a right $\mathcal{H}$-module

$$
\mathbb{T}(\pi):=\bigoplus_{\lambda \in \pi} x_{\lambda} \mathcal{H}
$$

and its centralizer algebra $\mathbb{S}(\pi):=\operatorname{End}_{\mathcal{H}}(\mathbb{T}(\pi))$; we let $\mathbb{S}(\pi)$ act on $\mathbb{T}(\pi)$ from the left.

It is obvious that $\mathbb{T}(\pi)$ has two bases $\left\{{ }^{\lambda} C_{w} \mid \lambda \in \pi, w \in{ }^{\lambda} W\right\}$ and $\left\{{ }^{\lambda} D_{w} \mid \lambda \in\right.$ $\left.\pi, w \in{ }^{\lambda} W\right\}$; we call them the Kazhdan-Lusztig basis and dual Kazhdan-Lusztig basis, respectively.

For each $m=\sum_{\lambda \in \pi} m_{\lambda} \in \mathbb{T}(\pi)$ with $m_{\lambda} \in x_{\lambda} \mathcal{H}$, we define $\bar{m} \in \mathbb{T}(\pi)$ to be $\sum_{\lambda \in \pi} \overline{m_{\lambda}}$. Also, for each $f \in \mathbb{S}(\pi)$, define $\bar{f} \in \mathbb{S}(\pi)$ by $\bar{f}(m)=\overline{f(\bar{m})}$ for all $m \in \mathbb{T}(\pi)$. This gives anti-linear automorphisms - on $\mathbb{T}(\pi)$ and $\mathbb{S}(\pi)$.

For each $\lambda \in \pi$, define $p_{\lambda} \in \mathbb{S}(\pi)$ to be the composite

$$
p_{\lambda}: \mathbb{T}(\pi) \rightarrow x_{\lambda} \mathcal{H} \hookrightarrow \mathbb{T}(\pi)
$$

of the projection and the inclusion. Clearly, $\left\{p_{\lambda} \mid \lambda \in \pi\right\}$ is a family of orthogonal idempotents with $\sum_{\lambda \in \pi} p_{\lambda}=\mathrm{id}_{\mathbb{T}(\pi)}$. Hence, we have a decomposition

$$
\mathbb{S}(\pi)=\bigoplus_{\lambda, \mu \in \pi} p_{\lambda} \mathbb{S}(\pi) p_{\mu}, \quad p_{\lambda} \mathbb{S}(\pi) p_{\mu}=\operatorname{Hom}_{\mathcal{H}}\left(x_{\mu} \mathcal{H}, x_{\lambda} \mathcal{H}\right)
$$


Take $f \in \operatorname{Hom}_{\mathcal{H}}\left(x_{\mu} \mathcal{H}, x_{\lambda} \mathcal{H}\right)$ arbitrarily. Since $x_{\mu} \mathcal{H}$ is generated (as a right $\mathcal{H}$ module) by $x_{\mu}$, the $f$ is determined by $f\left(x_{\mu}\right) \in x_{\lambda} \mathcal{H}$. Let us write

$$
f\left(x_{\mu}\right)=\sum_{w \in \in^{\lambda} W} c_{\lambda, w, \mu}(f) x_{\lambda} H_{w}, \quad \text { for some } c_{\lambda, w, \mu} \in \mathbf{A}_{\mathbb{Z}} .
$$

Lemma 7.1.2. Let $w \in{ }^{\lambda} W$ and $j \in I_{\mu}$ be such that $w<w s_{j}$. Then, we have

$$
c_{\lambda, w, \mu}(f)=q_{j} c_{\lambda, w s_{j}, \mu}(f) \text {. }
$$

Consequently, we have

$$
f\left(x_{\mu}\right)=\sum_{w \in{ }^{\lambda} W^{\mu}} \sum_{\substack{y \in W_{\mu} \\ w y \in^{\lambda} W}} q_{y}^{-1} c_{\lambda, w, \mu}(f) x_{\lambda} H_{w y},
$$

and hence, $f$ is determined by $\left(c_{\lambda, w, \mu}(f)\right)_{w \in{ }^{\lambda} W^{\mu}} \in \mathbf{A}_{\mathbb{Z}}^{\lambda} W^{\mu}$, where ${ }^{\lambda} W^{\mu}:={ }^{\lambda} W \cap$ $\left({ }^{\mu} W\right)^{-1}$.

Proof. We have

$$
\begin{aligned}
q_{j}^{-1} f\left(x_{\mu}\right)= & f\left(x_{\mu} H_{j}\right) \\
= & f\left(x_{\mu}\right) H_{j} \\
= & \sum_{\substack{w \in^{\lambda} W \\
w \epsilon_{j}<w}} c_{\lambda, w, \mu}(f) x_{\lambda}\left(H_{w s_{j}}+\left(q_{j}^{-1}-q_{j}\right) H_{w}\right)+\sum_{\substack{w \in^{\lambda} W \\
w s_{j}>w}} c_{\lambda, w, \mu}(f) x_{\lambda} H_{w s_{j}} \\
= & \sum_{\substack{w \in \in^{\lambda} W \\
w s_{j}<w}}\left(c_{\lambda, w s_{j}, \mu}(f)+\left(q_{j}^{-1}-q_{j}\right) c_{\lambda, w, \mu}(f)\right) x_{\lambda} H_{w}+\sum_{\substack{w \in \in^{\lambda} W \\
w s_{j}>w}} c_{\lambda, w s_{j}, \mu}(f) x_{\lambda} H_{w} .
\end{aligned}
$$

Comparing the coefficients of $x_{\lambda} H_{w}$, we obtain the assertion.

Conversely, given $\left(c_{\lambda, w, \mu}\right)_{w \in \in^{\lambda} W^{\mu}} \in \mathbf{A}_{\mathbb{Z}}^{\lambda} W^{\mu}$, there exists a unique

$$
g \in \operatorname{Hom}_{\mathcal{H}}\left(x_{\mu} \mathcal{H}, x_{\lambda} \mathcal{H}\right)
$$

such that $c_{\lambda, w, \mu}(g)=c_{\lambda, w, \mu}$ for all $w \in{ }^{\lambda} W^{\mu}$. Thus, we obtain an $\mathbf{A}_{\mathbb{Z}}$-linear isomorphism between $\mathbf{A}_{\mathbb{Z}}^{\lambda} W^{\mu}$ and $\operatorname{Hom}_{\mathcal{H}}\left(x_{\mu} \mathcal{H}, x_{\lambda} \mathcal{H}\right)$.

Lemma 7.1.3 ([DDPW08, Theorem 4.18]). Let $\lambda, \mu \in \pi$. For each $x \in{ }^{\lambda} W^{\mu}$, there exists a unique $J_{x} \subset\{0,1, \ldots, d-1\}$ such that the multiplication map

$$
W_{\lambda} \times\{x\} \times{ }^{J_{x}} W_{\mu} \rightarrow W_{\lambda} x W_{\mu} ;(u, x, v) \mapsto u x v
$$

is a bijection, where ${ }^{J_{x}} W_{\mu}:={ }^{J_{x}} W \cap W_{\mu}$. Moreover, we have $\ell(u x v)=\ell(u)+\ell(x)+$ $\ell(v)$ for all $u \in W_{\lambda}$ and $v \in{ }^{J_{x}} W_{\mu}$.

For $\lambda, \mu \in \pi$ and $x \in{ }^{\lambda} W^{\mu}$, define $\xi_{\lambda, x, \mu} \in \operatorname{Hom}_{\mathcal{H}}\left(x_{\mu} \mathcal{H}, x_{\lambda} \mathcal{H}\right)$ to be the one corresponding to $\left(\delta_{x, w} q_{x^{\prime}}\right)_{w \in \lambda W^{\mu}} \in \mathbf{A}_{\mathbb{Z}}^{\lambda} W^{\mu}$, where $x^{\prime} \in W_{\mu}$ is the longest element in ${ }^{J_{x}} W_{\mu}\left(J_{x}\right.$ is as in Lemma 7.1.3). Then, the next proposition is clear.

Proposition 7.1.4. $\left\{\xi_{\lambda, x, \mu} \mid \lambda, \mu \in \pi, x \in{ }^{\lambda} W^{\mu}\right\}$ forms a basis of $\mathbb{S}(\pi)$.

For each $\lambda, \mu \in \pi, x \in{ }^{\lambda} W^{\mu}$, set

$$
\eta_{\lambda, x, \mu}=q_{w_{\lambda} x x^{\prime}} \sum_{w \in W_{\lambda} x W_{\mu}} q_{w}^{-1} H_{w} .
$$


Lemma 7.1.5. Let $\lambda, \mu \in \pi, x \in{ }^{\lambda} W^{\mu}$.

(1) $\eta_{\lambda, x, \mu}^{b}=\eta_{\mu, x^{-1}, \lambda}$.

(2) $\xi_{\lambda, x, \mu}\left(x_{\mu}\right)=\eta_{\lambda, x, \mu}=\frac{1}{P_{\mu}} \eta_{\lambda, x, \mu} \cdot x_{\mu}=\frac{1}{P_{\lambda}} x_{\lambda} \cdot \eta_{\lambda, x, \mu}$.

(3) $\overline{\xi_{\lambda, e, \mu}}=\xi_{\lambda, e, \mu}$, where $e$ denotes the identity element of $W$.

Proof.

(1) By the definition of $x^{\prime}$, we have $y:=w_{\lambda} x x^{\prime}$ is the longest element in $W_{\lambda} x W_{\mu}$. Also, it is easily checked that the map $W \rightarrow W, w \mapsto w^{-1}$ gives a bijection $W_{\lambda} x W_{\mu} \rightarrow W_{\mu} x^{-1} W_{\lambda}$. Since this bijection preserves the length, $y^{-1}$ is the longest element in $W_{\mu} x^{-1} W_{\lambda}$. Then, we compute $\eta_{\lambda, x \mu}^{b}$ as follows:

$$
\begin{aligned}
\eta_{\lambda, x \mu}^{\mathrm{b}} & =q_{y} \sum_{w \in W_{\lambda} x W_{\mu}} q_{w}^{-1} H_{w^{-1}}=q_{y} \sum_{w \in W_{\mu} x^{-1} W_{\lambda}} q_{w^{-1}}^{-1} H_{w} \\
& =q_{y^{-1}} \sum_{w \in W_{\mu} x^{-1} W_{\lambda}} q_{w}^{-1} H_{w}=\eta_{\mu, x^{-1}, \lambda} .
\end{aligned}
$$

(2) By the definition of $\xi_{\lambda, x, \mu}$, we have

$$
\begin{aligned}
\xi_{\lambda, x, \mu}\left(x_{\mu}\right) & =\sum_{\substack{y \in W_{\mu} \\
x y \in \in^{\lambda} W}} q_{x^{\prime}} q_{y}^{-1} x_{\lambda} H_{x y} \\
& =\sum_{\substack{y \in W_{\mu} \\
x y \in \in^{\lambda} W}} \sum_{z \in W_{\lambda}} q_{x^{\prime}} q_{y}^{-1} q_{w_{\lambda}} q_{z}^{-1} H_{z x y} \quad \text { (by the definition of } x_{\lambda} \text { ) } \\
& =\sum_{w \in W_{\lambda} x W_{\mu}} q_{w_{\lambda}} q_{x} q_{x^{\prime}} q_{w}^{-1} H_{w}=\eta_{\lambda, x, \mu} .
\end{aligned}
$$

This proves the first equation. Next, we have

$$
\eta_{\lambda, x, \mu} \cdot x_{\mu}=\xi_{\lambda, x, \mu}\left(x_{\mu}^{2}\right)=P_{\mu} \xi_{\lambda, x, \mu}\left(x_{\mu}\right)=P_{\mu} \eta_{\lambda, x, \mu},
$$

which implies the second equality. Finally, the third equality follows from the fact that $\eta_{\lambda, x, \mu}=\xi_{\lambda, x, \mu}\left(x_{\mu}\right) \in x_{\lambda} \mathcal{H}$.

(3) It suffices to check that $\overline{\xi_{\lambda, e, \mu}}\left(x_{\nu}\right)=\xi_{\lambda, e, \mu}\left(x_{\nu}\right)$ for all $\nu \in \pi$. Only the non-trivial case is when $\nu=\mu$. Since we have

$$
\overline{\xi_{\lambda, e, \mu}}\left(x_{\mu}\right)=\overline{\xi_{\lambda, e, \mu}\left(x_{\mu}\right)}=\overline{\eta_{\lambda, e, \mu}},
$$

the problem is reduced to proving that $\eta_{\lambda, e, \mu}$ is fixed under the involution - . One can write

$$
\eta_{\lambda, e, \mu}=\sum_{w \in W_{\lambda} W_{\mu}} q_{w_{\lambda}} q_{e^{\prime}} q_{w}^{-1} H_{w}=x_{\lambda} \sum_{y \in \in^{\lambda} W_{\mu}} q_{e^{\prime}} q_{y}^{-1} H_{y} .
$$

On the other hand, we have

$$
x_{\lambda} x_{\mu}=x_{\lambda} x_{I_{\lambda} \cap I_{\mu}} \sum_{y \in \in^{\lambda} W_{\mu}} q_{e^{\prime}} q_{y}^{-1} H_{y}=P_{I_{\lambda} \cap I_{\mu}} x_{\lambda} \sum_{y \in \in^{\lambda} W_{\mu}} q_{e^{\prime}} q_{y}^{-1} H_{y} .
$$

Hence, we obtain

$$
\eta_{\lambda, e, \mu}=\frac{1}{P_{I_{\lambda} \cap I_{\mu}}} x_{\lambda} x_{\mu},
$$

which is invariant under $\odot$. Thus, the proof completes. 
Proposition 7.1.6. The linear map $b: \mathbb{S}(\pi) \rightarrow \mathbb{S}(\pi) ; \xi_{\lambda, x, \mu} \mapsto \xi_{\mu, x^{-1}, \lambda}$ defines an $\mathbf{A}_{\mathbb{Z}}$-algebra anti-automorphism on $\mathbb{S}(\pi)$.

Proof. We have to verify that $\left(\xi_{\lambda, x, \mu} \cdot \xi_{\kappa, y, \nu}\right)^{b}=\xi_{\nu, y^{-1}, \kappa} \cdot \xi_{\mu, x^{-1}, \lambda}$ for all $\lambda, \mu, \nu, \kappa$ and $x \in{ }^{\lambda} W^{\mu}, y \in{ }^{\kappa} W^{\nu}$. Since the both sides are equal to zero unless $\kappa=\mu$, we may assume that $\kappa=\mu$. Let us write

$$
\xi_{\lambda, x, \mu} \cdot \xi_{\mu, y, \nu}=\sum_{z \in^{\lambda} W^{\nu}} c_{z} \xi_{\lambda, z, \nu} \quad \text { for some } c_{z} \in \mathbf{A}_{\mathbb{Z}} .
$$

Applying the both sides to $x_{\nu} \in \mathbb{T}(\pi)$, by Lemma 7.1.5 (2), we obtain

$$
\frac{1}{P_{\mu}} \eta_{\lambda, x, \mu} \eta_{\mu, y, \nu}=\sum_{z \in \in^{\lambda} W^{\mu}} c_{z} \eta_{\lambda, z, \nu} .
$$

To prove the assertion, we compute as follows:

$$
\begin{aligned}
& \xi_{\nu, y^{-1}, \mu} \cdot \xi_{\mu, x^{-1}, \lambda}\left(x_{\lambda}\right)=\frac{1}{P_{\mu}} \eta_{\nu, y^{-1}, \mu} \cdot \eta_{\mu, x^{-1}, \lambda} \quad \text { (by Lemma 7.1.5 (2)) } \\
& =\left(\frac{1}{P_{\mu}} \eta_{\lambda, x, \mu} \cdot \eta_{\mu, y, \nu}\right)^{b} \quad \text { (by Lemma 7.1.5 (1) } \\
& \left.=\left(\sum_{z \in \in^{\lambda} W^{\nu}} c_{z} \eta_{\lambda, z, \nu}\right)^{b} \quad \text { (by equation (2) }\right) \\
& =\sum_{z \in \in^{\lambda} W^{\nu}} c_{z} \eta_{\nu, z^{-1}, \lambda} \quad \text { (by Lemma 7.1.5 (1)) } \\
& =\sum_{z \in^{\lambda} W^{\nu}} c_{z} \xi_{\nu, z^{-1}, \lambda}\left(x_{\lambda}\right) \quad \text { (by Lemma 7.1.5 (2)) } \\
& =\left(\xi_{\lambda, x, \mu} \cdot \xi_{\mu, y, \nu}\right)^{b}\left(x_{\lambda}\right) \quad \text { (by equation (10). }
\end{aligned}
$$

This shows that $\xi_{\nu, y^{-1}, \mu} \cdot \xi_{\mu, x^{-1}, \lambda}=\left(\xi_{\lambda, x, \mu} \cdot \xi_{\mu, y, \nu}\right)^{b}$, and hence, the proof completes.

Recall the bilinear form $\langle\cdot \mid \cdot\rangle_{\lambda}=\langle\cdot \mid \cdot\rangle_{I_{\lambda}}$ on $x_{\lambda} \mathcal{H}$ defined in Section 6.5,

Proposition 7.1.7. Let $\lambda, \mu \in \pi, m \in x_{\lambda} \mathcal{H}$, and $n \in x_{\mu} \mathcal{H}$. Then, for each $w \in{ }^{\lambda} W^{\mu}$, we have

$$
\left\langle m \mid \xi_{\lambda, w, \mu}(n)\right\rangle_{\lambda}=\left\langle\xi_{\lambda, w, \mu}^{b}(m) \mid n\right\rangle_{\mu}
$$

Proof. We compute as follows:

$$
\begin{array}{rlr}
\left\langle m \mid \xi_{\lambda, w, \mu}(n)\right\rangle_{\lambda} & =\frac{1}{P_{\lambda}}\left\langle m \mid \xi_{\lambda, w, \mu}(n)\right\rangle \\
& =\frac{1}{P_{\lambda} P_{\mu}}\left\langle m \mid \eta_{\lambda, w, \mu} n\right\rangle \quad \text { (by Lemma 7.1.5 (2)) } \\
& =\frac{1}{P_{\lambda} P_{\mu}}\left\langle\eta_{\mu, w^{-1}, \lambda} m \mid n\right\rangle \quad \text { (by Lemma 7.1.5 (1)) } \\
& =\frac{1}{P_{\mu}}\left\langle\xi_{\mu, w^{-1}, \lambda}(m) \mid n\right\rangle \quad \text { (by Lemma 7.1.5 (2)) } \\
& =\left\langle\xi_{\lambda, w, \mu}^{b}(m) \mid n\right\rangle_{\mu} .
\end{array}
$$

This proves the proposition. 
Define a bilinear form $\langle\cdot \mid \cdot\rangle_{\pi}$ on $\mathbb{T}(\pi)$ by $\langle m \mid n\rangle_{\pi}:=\delta_{\lambda, \mu}\langle m \mid n\rangle_{\lambda}$ for all $\lambda, \mu \in \pi$, $m \in x_{\lambda} \mathcal{H}, n \in x_{\mu} \mathcal{H}$.

Corollary 7.1.8. The two bases $\left\{{ }^{\lambda} C_{w} \mid \lambda \in \pi, w \in{ }^{\lambda} W\right\}$ and $\left\{{ }^{\lambda} D_{w_{\lambda} w w_{0}} \mid \lambda \in\right.$ $\left.\pi, w \in{ }^{\lambda} W\right\}$ of $\mathbb{T}(\pi)$ are dual to each other with respect to the bilinear form $\langle\cdot \mid \cdot\rangle_{\pi}$. Moreover, for all $m, n \in \mathbb{T}(\pi)$ and $x \in \mathbb{S}(\pi)$, we have $\langle m \mid x n\rangle_{\pi}=\left\langle x^{b} m \mid n\right\rangle_{\pi}$.

7.2. Cell representations. Let $X \in L(W)$ and $x \in X$. Set

$$
\begin{aligned}
& C_{\leq_{L} X}(\pi):=\bigoplus_{\lambda \in \pi} \bigoplus_{\substack{w \in \in^{\lambda} W \\
w_{\lambda} w \leq L}} \mathbf{A}_{\mathbb{Z}}{ }^{\lambda} C_{w}, \quad D_{\leq_{L}}(\pi):=\bigoplus_{\lambda \in \pi} \bigoplus_{\substack{w \in \in^{\lambda} W \\
w \leq \leq_{L} x}} \mathbf{A}_{\mathbb{Z}}{ }^{\lambda} D_{w}, \\
& C_{<{ }_{L} X}(\pi):=\bigoplus_{\lambda \in \pi} \bigoplus_{\substack{w \in \in^{\lambda} W \\
w_{\lambda} w<L}} \mathbf{A}_{\mathbb{Z}}{ }^{\lambda} C_{w}, \quad D_{\leq_{L}}(\pi):=\bigoplus_{\lambda \in \pi} \bigoplus_{\substack{w \in \in^{\lambda} W \\
w<L}} \mathbf{A}_{\mathbb{Z}^{\lambda}} D_{w}, \\
& C_{X}^{L}(\pi):=C_{\leq_{L} X}(\pi) / C_{<_{L} X}(\pi), \quad D_{X}^{L}(\pi):=D_{\leq_{L} X}(\pi) / D_{<_{L} X}(\pi) .
\end{aligned}
$$

Note that these objects are independent of the choice of $x \in X$. We denote the image of $m \in C_{\leq_{L} X}(\pi)$ (resp., $D_{\leq_{L} X}(\pi)$ ) under the quotient map $C_{\leq_{L} X}(\pi) \rightarrow$ $C_{X}^{L}(\pi)$ (resp., $\left.D_{\leq_{L} X}(\pi) \rightarrow D_{X}^{L}(\pi)\right)$ by $[m]_{X}$ (resp., $\left.[m]_{X}^{\prime}\right)$.

Proposition 7.2.1. Let $X \in L(W)$.

(1) $C_{\leq_{L} X}(\pi)$ is a $\mathbb{S}(\pi)$-submodule of $\mathbb{T}(\pi)$.

(2) $C_{<_{L} X}(\pi)$ is a $\mathbb{S}(\pi)$-submodule of $\mathbb{T}(\pi)$.

(3) $C_{X}^{L}(\pi)$ is a left $\mathbb{S}(\pi)$-module having a basis $\left\{\left[{ }^{\lambda} C_{w}\right]_{X} \mid \lambda \in \pi, w \in{ }^{\lambda} W \cap\right.$ $\left.w_{\lambda} X\right\}$. Here, $w_{\lambda} X:=\left\{w_{\lambda} x \mid x \in X\right\}$.

Proof. We will prove only (1) since the proof of (2) is similar to that of (1), and (3) follows from (1) and (2). Fix $x \in X$. In order to show that $C_{\leq_{L} X}(\pi)$ is a $\mathbb{S}(\pi)$-submodule, it suffices to verify that $\xi_{\lambda, y, \mu}{ }^{\mu} C_{w} \in C_{\leq_{L} X}(\pi)$ for all $\lambda, \mu \in \pi$, $y \in{ }^{\lambda} W^{\mu}$, and $w \in{ }^{\mu} W$ such that $w_{\mu} w \leq_{L} x$. By Proposition 6.5.3 and Lemma 7.1 .5 (2), we have

$$
\xi_{\lambda, y, \mu}{ }^{\mu} C_{w}=\xi_{\lambda, y, \mu} C_{w_{\mu} w}=\frac{1}{P_{\mu}} \eta_{\lambda, y, \mu} C_{w_{\mu} w} .
$$

Also, by Lemma 7.1.5 (2), we have $\eta_{\lambda, y, \mu}=\xi_{\lambda, y, \mu}\left(x_{\mu}\right) \in x_{\lambda} \mathcal{H}$; one can write $\eta_{\lambda, y, \mu}=x_{\lambda} H$ for some $H \in \mathcal{H}$. Then, $H C_{w_{\mu} w}$ is a linear combination of $C_{w^{\prime}}$, $w^{\prime} \leq_{L} w_{\mu} w\left(\leq_{L} x\right)$. Hence, by Proposition 6.5.5, $x_{\lambda} H C_{w_{\mu} w}$ is a linear combination of ${ }^{\lambda} C_{w^{\prime \prime}}$ for $w^{\prime \prime} \in{ }^{\lambda} W$ with $w_{\lambda} w^{\prime \prime} \leq_{L} w^{\prime}\left(\leq_{L} x\right)$. Therefore, we have $\xi_{\lambda, y, \mu}{ }^{\mu} C_{w}=$ $\frac{1}{P_{\mu}} \eta_{\lambda, y, \mu} C_{w_{\mu} w} \in \frac{1}{P_{\mu}} C_{\leq_{L} X}(\pi)$. However, since $\xi_{\lambda, y, \mu}{ }^{\mu} C_{w} \in x_{\lambda} \mathcal{H}=\bigoplus_{z \in{ }^{\lambda} W} \mathbf{A}_{\mathbb{Z}}{ }^{\lambda} C_{z}$, we conclude that $\xi_{\lambda, y, \mu}{ }^{\mu} C_{w} \in C_{\leq_{L} X}(\pi)$. This completes the proof.

Similarly, one can prove the following: $D_{\leq_{L} X}(\pi)$ and $D_{<_{L} X}(\pi)$ are $\mathbb{S}(\pi)$-submodules, and $D_{X}^{L}(\pi)$ is a left $\mathbb{S}(\pi)$-module having a basis $\left\{\left[{ }^{\lambda} D_{w}\right]_{X}^{\prime} \mid \lambda \in \pi, w \in\right.$ $\left.{ }^{\lambda} W \cap X\right\}$.

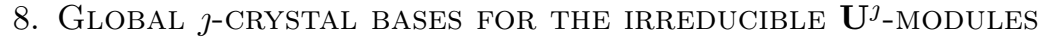

8.1. Surjection $\xi: \mathbf{U}^{\jmath} \rightarrow \mathbf{S}\left(\pi^{\jmath}\right)$. Let $\pi^{\jmath}=\left\{\lambda=\left(\lambda_{0}, \ldots, \lambda_{r}\right) \in \mathbb{Z}_{\geq 0}^{r+1} \mid \sum_{i=0}^{r} \lambda_{i}=\right.$ $d\}$. For $\lambda \in \pi^{\jmath}$, set $I_{\lambda}=\{0,1, \ldots, d-1\} \backslash\left\{\lambda_{0}, \lambda_{0,1}, \ldots, \lambda_{0, r-1}\right\}$, where $\lambda_{0, k}=$ $\sum_{i=0}^{k} \lambda_{i}$. 
Let $\mathbf{V}=\bigoplus_{i=-r}^{r} \mathbb{Q}(p, q) v_{i}$ be the vector representation of $\mathbf{U}$ with $v_{-r}$ a highest weight vector. Then, $\mathbf{V}^{\otimes d}$ has a basis $\left\{v_{i_{1}, \ldots, i_{d}}:=v_{i_{1}} \otimes \cdots \otimes v_{i_{d}} \mid-r \leq i_{1}, \ldots, i_{d} \leq\right.$ $r\} . \mathbf{H}:=\mathbb{Q}(p, q) \otimes_{\mathbf{A}_{\mathbb{Z}}} \mathcal{H}$ acts on $\mathbf{V}^{\otimes d}$ by

$$
\begin{aligned}
v_{i_{1}, \ldots, i_{d}} H_{0} & = \begin{cases}v_{-i_{1}, i_{2}, \ldots, i_{d}} & \text { if } i_{1}>0, \\
p^{-1} v_{i_{1}, \ldots, i_{d}} & \text { if } i_{1}=0, \\
v_{-i_{1}, i_{2}, \ldots, i_{d}}+\left(p^{-1}-p\right) v_{i_{1}, \ldots, i_{d}} & \text { if } i_{1}<0,\end{cases} \\
v_{i_{1}, \ldots, i_{d}} H_{j} & = \begin{cases}v_{\ldots, i_{j+1}, i_{j}, \ldots} & \text { if } i_{j}<i_{j+1}, \\
q^{-1} v_{i_{1}, \ldots, i_{d}} & \text { if } i_{j}=i_{j+1}, \\
v_{\ldots, i_{j+1}, i_{j}, \ldots}+\left(q^{-1}-q\right) v_{i_{1}, \ldots, i_{d}} & \text { if } i_{j}>i_{j+1}\end{cases}
\end{aligned}
$$

Then, it is easily seen that $\mathbf{V}^{\otimes d}$ is isomorphic to $\mathbf{T}\left(\pi^{\jmath}\right):=\mathbb{Q}(p, q) \otimes_{\mathbf{A}_{\mathbb{Z}}} \mathbb{T}\left(\pi^{\jmath}\right)$ as a right $\mathbf{H}$-module. Setting $\mathbf{S}\left(\pi^{\jmath}\right):=\mathbb{Q}(p, q) \otimes_{\mathbf{A}_{\mathbb{Z}}} \mathbb{S}\left(\pi^{\jmath}\right), \mathbf{V}^{\otimes d}$ becomes a left $\mathbf{S}\left(\pi^{\jmath}\right)$ module. By the double centralizer property between $\mathbf{U}^{3}$ and $\mathbf{H}$ on $\mathbf{V}^{\otimes d}$ (BW18a], BWW18), there exists a surjective algebra homomorphism $\xi: \mathbf{U}^{\jmath} \rightarrow \mathbf{S}\left(\pi^{\jmath}\right)$. In particular, every $\mathbf{S}\left(\pi^{\jmath}\right)$-modules are regarded as $\mathbf{U}^{\jmath}$-modules via $\xi$. In W17, it is proved that for each $\boldsymbol{\lambda} \in P^{\jmath}$, the irreducible highest weight module $L(\boldsymbol{\lambda})$ is isomorphic to $\mathbf{C}_{X}^{L}\left(\pi^{\jmath}\right):=\mathbb{Q}(p, q) \otimes_{\mathbf{A}_{\mathbb{Z}}} C_{X}^{L}\left(\pi^{\jmath}\right)$ for some $X \in L\left(W_{d}\right)$, where $d=|\boldsymbol{\lambda}|$.

For $i \in \mathbb{I}^{\jmath}$, we define two maps $\widetilde{e}_{i}, \widetilde{f}_{i}: \pi^{\jmath} \rightarrow \pi^{\jmath} \sqcup\{0\}$, where 0 denotes a formal symbol, as follows. Let $\lambda=\left(\lambda_{0}, \ldots, \lambda_{r}\right) \in \pi^{\jmath}$. Then, we set

$$
\widetilde{e}_{i} \lambda= \begin{cases}\left(\lambda_{0}, \ldots, \lambda_{i-2}, \lambda_{i-1}+1, \lambda_{i}-1, \lambda_{i+1}, \ldots, \lambda_{r}\right) & \text { if } \lambda_{i}>0 \\ 0 & \text { if } \lambda_{i}=0\end{cases}
$$

and

$$
\widetilde{f}_{i} \lambda= \begin{cases}\left(\lambda_{0}, \ldots, \lambda_{i-2}, \lambda_{i-1}-1, \lambda_{i}+1, \lambda_{i+1}, \ldots, \lambda_{r}\right) & \text { if } \lambda_{i-1}>0 \\ 0 & \text { if } \lambda_{i-1}=0\end{cases}
$$

By convention, we set $\xi_{\lambda, x, \mu}=0$ if $\lambda=0$ or $\mu=0$.

Proposition 8.1.1. For $i \in \mathbb{I}^{\jmath}$, we have

$$
\begin{aligned}
& \xi\left(e_{i}\right)=\sum_{\lambda \in \pi^{\jmath}} \xi_{\widetilde{e}_{i}(\lambda), e, \lambda}, \\
& \xi\left(f_{i}\right)=\sum_{\lambda \in \pi^{j}} \xi_{\widetilde{f}_{i}(\lambda), e, \lambda} .
\end{aligned}
$$

Proof. We prove only the statement for $f_{1}$; the proofs for $f_{i}, i \neq 1$ and for $e_{i}$ are similar. Recall the comultiplication $\Delta$ of $\mathbf{U}$; we have

$$
\Delta^{(d-1)}\left(E_{i}\right)=\sum_{k=1}^{d} 1^{\otimes k-1} \otimes E_{i} \otimes\left(K_{i}^{-1}\right)^{\otimes d-k}, \Delta^{(d-1)}\left(F_{i}\right)=\sum_{k=1}^{d} K_{i}^{\otimes d-k} \otimes F_{i} \otimes 1^{\otimes k-1} .
$$


Then, we compute as

$$
\begin{aligned}
f_{1} v_{\lambda}= & p q^{-1} q^{\lambda_{0}} \sum_{k=1}^{\lambda_{0}} q^{\lambda_{0}-k} v_{0^{\lambda_{0}-k}, 1,0^{k-1}, 1^{\lambda_{1}}, \ldots, r^{\lambda_{r}}} \\
& +\sum_{k=1}^{\lambda_{0}} q^{\lambda_{0}-k} v_{0^{k-1},-1,0^{\lambda_{0}-k}, 1^{\lambda_{1}}, \ldots, r^{\lambda_{r}}} \\
= & p q^{\lambda_{0}-1} \sum_{k=1}^{\lambda_{0}} q^{\lambda_{0}-k} v_{\widetilde{f}_{1}(\lambda)} H_{\lambda_{0}-1} \cdots H_{\lambda_{0}-(k-1)} \\
& +\sum_{k=1}^{\lambda_{0}} q^{\lambda_{0}-k} v_{\widetilde{f}_{1}(\lambda)} H_{\lambda_{0}-1} \cdots H_{1} H_{0} H_{1} \cdots H_{k-1} \\
= & p q^{2\left(\lambda_{0}-1\right)} \sum_{k=1}^{\lambda_{0}} q^{-k+1} v_{\widetilde{f}_{1}(\lambda)} H_{\lambda_{0}-1} \cdots H_{\lambda_{0}-(k-1)} \\
& +p q^{2\left(\lambda_{0}-1\right)} \sum^{-1} q^{-\left(\lambda_{0}+k-2\right)} v_{\widetilde{f}_{1}(\lambda)} H_{\lambda_{0}-1} \cdots H_{1} H_{0} H_{1} \cdots H_{k-1} \\
= & \xi_{\widetilde{f}_{1}(\lambda), e, \lambda}\left(v_{\lambda}\right) .
\end{aligned}
$$

This proves the assertion.

Here are immediate consequences.

Corollary 8.1.2. Let $x \in \mathbf{U}^{\jmath}$. Then, $\xi\left(\sigma^{\jmath}(x)\right)=\xi(x)^{b}, \xi\left(\psi^{\jmath}(x)\right)=\overline{\xi(x)}$.

Corollary 8.1.3. The bilinear form $\langle\cdot \mid \cdot\rangle_{\pi^{\jmath}}$ of $\mathbf{T}\left(\pi^{\jmath}\right)$ satisfies

$$
\langle x m \mid n\rangle_{\pi^{\jmath}}=\left\langle m \mid \sigma^{\jmath}(x) n\right\rangle_{\pi^{\jmath}}
$$

for all $x \in \mathbf{U}^{\jmath}, m, n \in \mathbf{T}\left(\pi^{\jmath}\right)$.

8.2. Global $\jmath$-crystal basis of irreducible $\mathbf{U}^{\jmath}$-module. Let $X \in L(W)$. Then, $\mathbf{C}_{X}^{L}\left(\pi^{\jmath}\right) \simeq L(\boldsymbol{\lambda})$ for some $\boldsymbol{\lambda} \in P^{\jmath}$. Since $L(\boldsymbol{\lambda})$ is a highest weight module, there exists a unique $\lambda \in \pi^{\jmath}$ and $w \in{ }^{\lambda} W$ such that $\left[{ }^{\lambda} C_{w}\right]_{X} \in \mathbf{C}_{X}^{L}\left(\pi^{\jmath}\right)$ is a highest weight vector.

Recall the isomorphism $D_{X w_{0}}^{L} \simeq C_{X}^{L}$ of left $\mathcal{H}$-modules. Set $\mathbf{C}_{X}^{L}:=\mathbb{Q}(p, q) \otimes_{\mathbf{A}_{\mathbb{Z}}}$ $C_{X}^{L}$, and define $\mathbf{D}_{X w_{0}}^{L}$ and $\mathbf{D}_{X w_{0}}^{L}\left(\pi^{J}\right)$ similarly. Then, we have

$$
\mathbf{D}_{X w_{0}}^{L}\left(\pi^{\jmath}\right) \simeq \mathbf{T}\left(\pi^{\jmath}\right) \otimes_{\mathbf{H}} \mathbf{D}_{X w_{0}}^{L} \simeq \mathbf{T}\left(\pi^{\jmath}\right) \otimes_{\mathbf{H}} \mathbf{C}_{X}^{L} \simeq \mathbf{C}_{X}^{L}\left(\pi^{\jmath}\right)
$$

as left $\mathbf{U}^{\jmath}$-modules. Hence, $\left[{ }^{\lambda} D_{w_{\lambda} w w_{0}}\right]_{X w_{0}}^{\prime} \in \mathbf{D}_{X w_{0}}^{L}\left(\pi^{\jmath}\right)$ is also a highest weight vector. Thus, we obtain two isomorphisms

$$
\begin{aligned}
& \varphi_{C}: L(\boldsymbol{\lambda}) \rightarrow \mathbf{C}_{X}^{L}\left(\pi^{\jmath}\right) ; v_{\boldsymbol{\lambda}} \mapsto\left[{ }^{\lambda} C_{w}\right]_{X}, \\
& \varphi_{D}: L(\boldsymbol{\lambda}) \rightarrow \mathbf{D}_{X w_{0}}^{L}\left(\pi^{\jmath}\right) ; v_{\boldsymbol{\lambda}} \mapsto\left[{ }^{\lambda} D_{w_{\lambda} w w_{0}}\right]_{X w_{0}}^{\prime}
\end{aligned}
$$

of $\mathbf{U}^{3}$-modules, where $v_{\boldsymbol{\lambda}} \in L(\boldsymbol{\lambda})$ is a fixed highest weight vector.

Definition 8.2.1. Let $\boldsymbol{\lambda} \in P^{J}$ and $v_{\boldsymbol{\lambda}} \in L(\boldsymbol{\lambda})$ be a highest weight vector. Define the bilinear form $(\cdot, \cdot)_{1}$ on $L(\boldsymbol{\lambda})$ by $\left(v_{\boldsymbol{\lambda}}, v_{\boldsymbol{\lambda}}\right)_{1}=1$ and $(x m, n)_{1}=\left(n, \sigma^{\jmath}(x) n\right)_{1}$ for all $x \in \mathbf{U}^{\jmath}, m, n \in L(\boldsymbol{\lambda})$.

Proposition 8.2.2. Let $\boldsymbol{\lambda} \in P^{J}$. Then, the bilinear form $(\cdot, \cdot)_{1}$ is nondegenerate. 
Proof. For $m, n \in L(\boldsymbol{\lambda})$, set $(m, n):=\left\langle\varphi_{C}(m) \mid \varphi_{D}(n)\right\rangle_{\pi^{\jmath}}$. Then, we have

$$
\left(v_{\boldsymbol{\lambda}}, v_{\boldsymbol{\lambda}}\right)=\left\langle\left[{ }^{\lambda} C_{w}\right]_{X} \mid\left[{ }^{\lambda} D_{w_{\lambda} w w_{0}}\right]_{X w_{0}}^{\prime}\right\rangle_{\pi^{\jmath}}=1
$$

and

$$
(x m, n)=\left\langle x \varphi_{C}(m) \mid \varphi_{D}(m)\right\rangle_{\pi^{\jmath}}=\left\langle\varphi_{C}(m) \mid \sigma^{\jmath}(x) \varphi_{D}(n)\right\rangle_{\pi^{\jmath}}=\left(m, \sigma^{\jmath}(x) n\right) .
$$

Hence, we have $(\cdot, \cdot)=(\cdot, \cdot)_{1}$. Then, it is clear that $\left\{\varphi_{C}^{-1}\left(\left[{ }^{\mu} C_{y}\right]_{X}\right) \mid \mu \in \pi^{\jmath}, y \in\right.$ $\left.{ }^{\mu} W \cap w_{\mu} X\right\}$ and $\left\{\varphi_{D}^{-1}\left(\left[{ }^{\mu} D_{w_{\mu} y w_{0}}\right]_{X w_{0}}^{\prime}\right) \mid \mu \in \pi^{\jmath}, y \in{ }^{\mu} W \cap X w_{0}\right\}$ form bases which are dual to each other with respect to $(\cdot, \cdot)_{1}$. This proves the proposition.

Recall that the set $\left\{(\mu, y) \mid \mu \in \pi^{\jmath}, y \in{ }^{\mu} W \cap w_{\mu} X\right\}$ is identical to $\mathcal{B}(\boldsymbol{\lambda})$. For each $b \in \mathcal{B}(\boldsymbol{\lambda})$, set

$$
G_{\text {low }}^{J}(b):=\varphi_{C}^{-1}\left(\left[{ }^{\mu} C_{y}\right]_{X}\right), \quad G_{\mathrm{up}}^{J}(b):=\varphi_{D}^{-1}\left(\left[{ }^{\mu} D_{w_{\mu} y w_{0}}\right]_{X w_{0}}^{\prime}\right),
$$

where $(\mu, y)$ is the pair corresponding to $b$. Then, $G_{\text {low }}^{J}(\boldsymbol{\lambda}):=\left\{G_{\text {low }}^{\text {low }}(b) \mid b \in \mathcal{B}(\boldsymbol{\lambda})\right\}$ and $G_{\text {up }}^{j}(\boldsymbol{\lambda}):=\left\{G_{\text {up }}^{\jmath}(b) \mid b \in \mathcal{B}(\boldsymbol{\lambda})\right\}$ are bases of $L(\boldsymbol{\lambda})$.

Definition 8.2.3. Let $\boldsymbol{\lambda} \in P^{J}(d)$, and $v_{\boldsymbol{\lambda}} \in L(\boldsymbol{\lambda})$ be a highest weight vector. Define a bilinear form $(\cdot, \cdot)_{2}$ on $L(\boldsymbol{\lambda})$, and a $\psi^{3}$-involution $\psi_{\boldsymbol{\lambda}}^{\mathrm{J}}$ on $L(\boldsymbol{\lambda})$ by

$$
\begin{aligned}
& \left(v_{\boldsymbol{\lambda}}, v_{\boldsymbol{\lambda}}\right)_{2}=1,(x m, n)_{2}=\left(m, \tau^{\jmath}(x) n\right)_{2} \quad \text { for all } x \in \mathbf{U}^{\jmath}, m, n \in L(\boldsymbol{\lambda}), \\
& \psi_{\boldsymbol{\lambda}}^{\jmath}\left(v_{\boldsymbol{\lambda}}\right)=v_{\boldsymbol{\lambda}} .
\end{aligned}
$$
$\mathcal{B}(\boldsymbol{\lambda})$.

Let $(\mathcal{L}(\boldsymbol{\lambda}), \mathcal{B}(\boldsymbol{\lambda}))$ be the unique -crystal basis of $L(\boldsymbol{\lambda})$ such that $v_{\boldsymbol{\lambda}}+q \mathcal{L}(\boldsymbol{\lambda}) \in$

Theorem 8.2.4. Let $\boldsymbol{\lambda} \in P^{J}(d)$. Then, the following hold.

(1) $\psi_{\boldsymbol{\lambda}}^{\mathrm{J}}\left(G_{\text {low }}^{\text {J }}(b)\right)=G_{\text {low }}^{\text {J }}(b)$ for all $b \in \mathcal{B}(\boldsymbol{\lambda})$.

(2) $\psi_{\boldsymbol{\lambda}}^{\jmath}\left(G_{\mathrm{up}}^{\jmath}(b)\right)=G_{\mathrm{up}}^{\jmath}(b)$ for all $b \in \mathcal{B}(\boldsymbol{\lambda})$.

(3) $G_{\text {low }}^{J}(\boldsymbol{\lambda})$ and $G_{\text {up }}^{\text {u }}(\boldsymbol{\lambda})$ are dual bases with respect to $(\cdot, \cdot)_{1}$.

(4) $\mathcal{L}(\boldsymbol{\lambda})=\left\{m \in L(\boldsymbol{\lambda}) \mid(m, m)_{2} \in \mathbf{A}_{0}\right\}$. Consequently, $(\cdot, \cdot)_{2}$ induces the bilinear form $(\cdot, \cdot)_{0}$ on $\mathcal{L}(\boldsymbol{\lambda}) / q \mathcal{L}(\boldsymbol{\lambda})$ defined by $(m+q \mathcal{L}(\boldsymbol{\lambda}), n+q \mathcal{L}(\boldsymbol{\lambda}))_{0}:=$ $\lim _{q \rightarrow 0}\left(\lim _{p \rightarrow 0}(m, n)_{2}\right)$.

(5) $\left\{G_{\text {low }}^{J}(b) \mid b \in \mathcal{B}(\boldsymbol{\lambda})\right\}$ forms an almost orthonormal basis with respect to $(\cdot, \cdot)_{2}$, i.e., we have $\left(G_{\text {low }}^{J}(b), G_{\text {low }}^{J}\left(b^{\prime}\right)\right)_{2} \in \delta_{b, b^{\prime}}+q \mathbf{A}_{0}$ for all $b, b^{\prime} \in \mathcal{B}(\boldsymbol{\lambda})$.

(6) $\left(b, b^{\prime}\right)_{0}=\delta_{b, b^{\prime}}$ for all $b, b^{\prime} \in \mathcal{B}(\boldsymbol{\lambda})$.

(7) Let $L(\boldsymbol{\lambda})_{\mathbf{A}}$ be the $\mathbf{A}$-span of $G_{\text {low }}^{J}(\boldsymbol{\lambda})$. Then, $\left(\mathcal{L}(\boldsymbol{\lambda}), L(\boldsymbol{\lambda})_{\mathbf{A}}, \psi_{\boldsymbol{\lambda}}^{J}(\mathcal{L}(\boldsymbol{\lambda}))\right)$ is balanced. Moreover, the global basis associated to $\mathcal{B}(\boldsymbol{\lambda})$ is $\left\{G_{\text {low }}^{\jmath}(b) \mid b \in\right.$ $\mathcal{B}(\boldsymbol{\lambda})\}$. In particular, $L(\boldsymbol{\lambda})$ has a global $\mathrm{\jmath}$-crystal basis.

Proof. Items (1) and (2) are obvious from the definition of $G_{\text {low }}^{J}(b)$ and $G_{\mathrm{up}}^{\jmath}(b)$. Item (3) follows from the proof of Proposition 8.2.2

To prove the rest, observe that $L(\boldsymbol{\lambda})$ is realized as a subquotient of $\mathbf{V}^{\otimes d}$ by using Kazhdan-Lusztig basis elements. To be precise, let $X \in P^{J}$ be such that $L(\boldsymbol{\lambda}) \simeq \mathbf{C}_{X}^{L}\left(\pi^{\jmath}\right)$ and $x \in X$. Then,

$$
\mathbf{C}_{X}^{L}\left(\pi^{\jmath}\right)=\frac{\operatorname{Span}_{\mathbb{Q}(p, q)}\left\{{ }^{\lambda} C_{w} \mid \lambda \in \pi^{\jmath}, w \in{ }^{\lambda} W, w_{\lambda} w \leq_{L} x\right\}}{\operatorname{Span}_{\mathbb{Q}(p, q)}\left\{{ }^{\mu} C_{y} \mid \mu \in \pi^{\jmath}, y \in{ }^{\mu} W, w_{\mu} y<_{L} x\right\}} .
$$

Then, items (4)-(6) follows from the proof of [W17, Proposition 7.4.4]. To prove item (7), it suffices to show that $L(\boldsymbol{\lambda})_{\mathbf{A}}$ is a $\mathbf{U}_{\mathbf{A}}^{\jmath}$-module. It follows from the fact that the $\mathbf{A}$-submodule of $\mathbf{V}^{\otimes d}$ spanned by the Kazhdan-Lusztig basis is a $\mathbf{U}_{\mathbf{A}}$-module, and that $\mathbf{U}_{\mathbf{A}}^{\jmath} \subset \mathbf{U}_{\mathbf{A}}$. 


\section{BASIC PROPERTIES OF GLOBAL CRYSTAL BASES}

9.1. Global crystal bases. In this subsection, we exposite some basic properties concerning global crystal bases of $\mathbf{U}$-modules in $\mathcal{O}_{\text {int }}$. Let $M \in \mathcal{O}_{\text {int }},(\mathcal{L}, \mathcal{B})$ a crystal basis of $M, \psi_{M}$ a $\psi$-involution, and $M_{\mathbf{A}}$ a $\mathbf{U}_{\mathbf{A}}$-submodule of $M$. Suppose that $M$ has a global basis $G(\mathcal{B})$ with the associated balanced triple $\left(\mathcal{L}, M_{\mathbf{A}}, \psi_{M}(\mathcal{L})\right)$.

Proposition 9.1.1 ([K93]). Let $i \in \mathbb{I}, b \in B$ and $m \in \mathbb{Z}_{\geq 0}$. Then, we have the following.

(1) $\sum_{n \geq m} F_{i}^{(n)} M_{\mathbf{A}}=\bigoplus_{\substack{b^{\prime} \in \mathcal{B} \\ \varepsilon_{i}\left(b^{\prime}\right) \geq m}} \mathbf{A} G^{\jmath}\left(b^{\prime}\right)$.

(2) $\sum_{n \geq m} E_{i}^{(n)} M_{\mathbf{A}}=\bigoplus_{\substack{b^{\prime} \in \mathcal{B} \\ \varphi_{i}\left(b^{\prime}\right) \geq m}} \mathbf{A} G^{\jmath}\left(b^{\prime}\right)$.

(3) $F_{i} G^{\jmath}(b)=\left[\varepsilon_{i}(b)+1\right] G^{\jmath}\left(\widetilde{F}_{i} b\right)+\sum_{\varepsilon_{i}\left(b^{\prime}\right)>\varepsilon_{i}(b)+1}^{b^{\prime} \in \mathcal{B}} \varphi_{b^{\prime}, b}^{(i)} G^{\jmath}\left(b^{\prime}\right)$ for some $\varphi_{b^{\prime}, b}^{(i)} \in$ $q^{2-\varepsilon_{i}\left(b^{\prime}\right)} \mathbb{Q}[q]$.

(4) $E_{i} G^{\jmath}(b)=\left[\varphi_{i}(b)+1\right] G^{\jmath}\left(\widetilde{E}_{i} b\right)+\sum_{\substack{b^{\prime} \in \mathcal{B} \\ \varphi_{i}\left(b^{\prime}\right)>\varphi_{i}(b)+1}} \varepsilon_{b^{\prime}, b}^{(i)} G^{\jmath}\left(b^{\prime}\right)$ for some $\varepsilon_{b^{\prime}, b}^{(i)} \in$ $q^{2-\varphi_{i}\left(b^{\prime}\right)} \mathbb{Q}[q]$.

For $\lambda \in P(M)$, set $I_{\lambda}(M)$ to be the sum of submodules of $M$ isomorphic to $L(\lambda)$. Also, we set

$$
\begin{aligned}
& W_{\succeq \lambda}(M):=\sum_{\mu \succeq \lambda} I_{\mu}(M), \\
& W_{\succ \lambda}(M):=\sum_{\mu \succ \lambda} I_{\mu}(M), \\
& W_{\lambda}(M):=W_{\succeq \lambda}(M) / W_{\succ \lambda}(M) .
\end{aligned}
$$

Theorem 9.1.2 ([K93], [L10]). Let $M, \mathcal{L}, \mathcal{B}, M_{\mathbf{A}}$ be as above. Then, for each $\lambda \in P(M)$, the following hold:

(1) $W_{\succeq \lambda}(M)$ has a global crystal basis $W_{\succ \lambda}(G(\mathcal{B})):=\{G(b) \mid I(b) \succeq \lambda\}$ with the associated balanced triple $\left(W_{\succeq \lambda}(\overline{\mathcal{L}}), W_{\succeq \lambda}\left(M_{\mathbf{A}}\right), W_{\succeq \lambda}\left(\psi_{M}(\mathcal{L})\right)\right)$, where $W_{\succeq \lambda}(\mathcal{L}):=W_{\succeq \lambda}(M) \cap \mathcal{L}$, and so on.

(2) $W_{\succ \lambda}(M)$ has a global crystal basis $W_{\succ \lambda}(G(\mathcal{B})):=\{G(b) \mid I(b) \succ \lambda\}$ with the associated balanced triple $\left(W_{\succ \lambda}(\mathcal{L}), W_{\succ \lambda}\left(M_{\mathbf{A}}\right), W_{\succ \lambda}\left(\psi_{M}(\mathcal{L})\right)\right)$, where $W_{\succ \lambda}(\mathcal{L}):=W_{\succ \lambda}(M) \cap \mathcal{L}$, and so on.

(3) $W_{\lambda}(M)$ has a global crystal basis $W_{\lambda}(G(\mathcal{B})):=\left\{G(b)+W_{\succ \lambda}(M) \mid I(b)=\lambda\right\}$ with the associated balanced triple $\left(W_{\lambda}(\mathcal{L}), W_{\lambda}\left(M_{\mathbf{A}}\right), W_{\lambda}\left(\psi_{M}(\mathcal{L})\right)\right)$, where $W_{\lambda}(\mathcal{L}):=W_{\succeq \lambda}(\mathcal{L}) / W_{\succ \lambda}(\mathcal{L})$, and so on.

(4) There exists a U-module isomorphism $\xi: L(\lambda)^{\oplus m_{\lambda}} \rightarrow W_{\lambda}(M)$ which induces an isomorphism

$$
\left(\mathcal{L}(\lambda)^{\oplus m_{\lambda}},\left(L(\lambda)_{\mathbf{A}}\right)^{\oplus m_{\lambda}}, \psi_{\lambda}(\mathcal{L}(\lambda))^{\oplus m_{\lambda}}\right) \simeq\left(W_{\lambda}(\mathcal{L}), W_{\lambda}\left(M_{\mathbf{A}}\right), W_{\lambda}\left(\psi_{M}(\mathcal{L})\right)\right),
$$

where $m_{\lambda}:=\operatorname{dim} \operatorname{Hom}_{\mathbf{U}}(L(\lambda), M)$ denotes the multiplicity of $L(\lambda)$ in $M$.

Remark 9.1.3. By replacing $P(M)$ with $P^{\jmath}(M)$ and $\preceq$ with $\unlhd$, the same result holds for integrable modules over $\mathbf{U}(\mathfrak{l})$ with global crystal bases.

9.2. J-canonical bases. Let $M \in \mathcal{O}_{\text {int }}$ be a based $\mathbf{U}$-module with a crystal basis $(\mathcal{L}, \mathcal{B})$, a global crystal basis $G(\mathcal{B})$, a $\psi$-involution $\psi_{M}$, and a balanced triple $\left(\mathcal{L}, M_{\mathbf{A}}, \psi_{M}(\mathcal{L})\right)$. Set $\psi_{M}^{J}:=\Upsilon \circ \psi_{M}$. We denote by $G^{\jmath}(\mathcal{B})$ the associated $\jmath$-canonical 
basis. Recall that $\psi_{M}^{\jmath}$ is a $\psi^{\jmath}$-involution on $M$, and $\left(\mathcal{L}, M_{\mathbf{A}}, \psi_{M}^{\jmath}(\mathcal{L})\right)$ is a balanced triple with the associated global basis $G^{\jmath}(\mathcal{B})$.

Lemma 9.2.1. Let $b \in \mathcal{B}$. Let us write as

$$
G^{\jmath}(b)=G(b)+\sum_{\substack{b^{\prime} \in \mathcal{B} \\ \mathrm{wt}^{\jmath}\left(b^{\prime}\right)=\mathrm{wt}^{\jmath}(b) \text { and } \operatorname{wt}\left(b^{\prime}\right)<\mathrm{wt}(b)}} c_{b^{\prime}, b} G\left(b^{\prime}\right)
$$

for some $c_{b^{\prime}, b} \in q \mathbf{A}_{0} \cap \mathbf{A}$. Then, we have $c_{b^{\prime}, b}=0$ unless

$$
I^{\jmath}(b) \unlhd I^{\jmath}\left(b^{\prime}\right) \text { or }\left|I^{\jmath}\left(b^{\prime}\right)^{-}\right|<\left|I^{\jmath}(b)^{-}\right| .
$$

Proof. By the construction of $G^{\jmath}(b)$, it suffices to show that $\psi_{M}^{J}(G(b))$ is a linear combination of $G\left(b^{\prime}\right)$ with $b^{\prime}$ satisfying (3). Since $\psi_{M}^{J}(G(b))=\Upsilon G(b) \in \mathbf{U}^{-} G(b)$, it suffices to show that for each $l \in \mathbb{Z}_{\geq 0}$ and $i_{1}, \ldots, i_{l} \in \mathbb{I}$, we have

$$
F_{i_{l}} \cdots F_{i_{1}} G(b) \in \operatorname{Span}_{\mathbb{Q}(p, q)}\left\{G\left(b^{\prime}\right) \mid b^{\prime} \text { satisfies condition (3) }\right\} .
$$

We prove it by induction on $l$. When $l=0$, there are nothing to prove. So, assume that $l>0$ and that $F_{i_{l-1}} \cdots F_{i_{1}} G(b) \in \operatorname{Span}_{\mathbb{Q}(p, q)}\left\{G\left(b^{\prime}\right) \mid b^{\prime}\right.$ satisfies condition (3) $\}$ for all $i_{1}, \ldots, i_{l-1} \in \mathbb{I}$. If $i_{l} \neq \underline{1}$, then, by Remark 9.1.3, we have

$$
F_{i_{l}} G\left(b^{\prime}\right) \in \operatorname{Span}_{\mathbb{Q}(p, q)}\left\{G\left(b^{\prime \prime}\right) \mid I^{\jmath}\left(b^{\prime}\right) \unlhd I^{\jmath}\left(b^{\prime \prime}\right)\right\}
$$

for all $b^{\prime}$ satisfying condition (3). Since $\left|I^{\jmath}\left(b^{\prime \prime}\right)^{-}\right|=\left|I^{\jmath}\left(b^{\prime}\right)^{-}\right|$for all $b^{\prime \prime}$ with $I^{\jmath}\left(b^{\prime}\right) \unlhd$ $I^{\jmath}\left(b^{\prime \prime}\right), b^{\prime \prime}$ satisfies condition (3).

If $i_{l}=\underline{1}$, then $\operatorname{wt}\left(F_{i_{l}} G\left(b^{\prime}\right)\right)=\operatorname{wt}\left(G\left(b^{\prime}\right)\right)-\alpha_{\underline{1}}$. This immediately implies that $F_{i_{l}} G\left(b^{\prime}\right) \in \operatorname{Span}_{\mathbb{Q}(p, q)}\left\{G\left(b^{\prime \prime}\right)|| I^{\jmath}\left(b^{\prime \prime}\right)^{-}|<| I^{J}\left(b^{\prime}\right)^{-} \mid\right\}$. Therefore, $F_{i_{l}} \cdots F_{i_{1}} G(b)$ is a linear combination of $G\left(b^{\prime}\right)$ with $\left|I^{J}\left(b^{\prime}\right)^{-}\right|<\left|I^{\jmath}(b)^{-}\right|$. Thus, the proof completes.

Proposition 9.2.2. Let $b \in \mathcal{B}$ and $i \in \mathbb{I}^{\jmath} \backslash\{1\}$. Then, we have

$$
\begin{aligned}
& e_{i} G^{\jmath}(b)=\left[\varphi_{\underline{i}}(b)+1\right] G^{\jmath}\left(\widetilde{E}_{\underline{i}} b\right)+\sum_{\substack{\widetilde{b}^{\prime} \in \mathcal{B} \backslash\left\{\widetilde{E}_{\underline{i}} b\right\} \\
\mathrm{wt}^{\jmath}\left(b^{\prime}\right)=\mathrm{wt}^{\jmath}(b)+\gamma_{i} \text { and } \underline{\operatorname{wt}}\left(b^{\prime}\right) \leq \mathrm{wt}(b)+\alpha_{\underline{i}}}} e_{b^{\prime}, b}^{(i)} G^{J}\left(b^{\prime}\right), \\
& f_{i} G^{\jmath}(b)=\left[\varphi_{-\underline{i}}(b)+1\right] G^{\jmath}\left(\widetilde{E}_{-\underline{i}} b\right)+\sum_{\substack{b^{\prime} \in \mathcal{B} \backslash\left\{\widetilde{E}_{-\underline{i}} b\right\} \\
\mathrm{wt}^{\jmath}\left(b^{\prime}\right)=\mathrm{wt}^{\jmath}(b)-\gamma_{i} \text { and } \operatorname{wt}\left(b^{\prime}\right) \leq \mathrm{wt}(b)+\alpha_{-i}}} f_{b^{\prime}, b}^{(i)} G^{\jmath}\left(b^{\prime}\right)
\end{aligned}
$$

for some $e_{b^{\prime}, b}^{(i)}, f_{b^{\prime}, b}^{(i)} \in \mathbf{A} . \quad$ Moreover, $e_{b^{\prime}, b}^{(i)}=f_{b^{\prime}, b}^{(i)}=0$ unless $I^{\jmath}(b) \unlhd I^{\jmath}\left(b^{\prime}\right)$ or $\left|I^{\jmath}\left(b^{\prime}\right)^{-}\right|<\left|I^{\jmath}(b)^{-}\right|$.

Proof. We prove the assertion only for $e_{i}$; the proof for $f_{i}$ is similar. By Lemma 9.2.1, we can write

$$
G^{\jmath}(b)=G(b)+\sum_{b^{\prime} \in \mathcal{B} \backslash\{b\}} c_{b^{\prime}, b} G\left(b^{\prime}\right)
$$

for some $c_{b^{\prime}, b} \in \mathbf{A}$ such that $c_{b^{\prime}, b}=0$ unless $I^{\jmath}(b) \unlhd I^{\jmath}\left(b^{\prime}\right)$ or $\left|I^{\jmath}\left(b^{\prime}\right)^{-}\right|<\left|I^{\jmath}(b)^{-}\right|$. Since $e_{i} \in U_{q}(\mathfrak{l})$, it holds that

$$
e_{i} G^{\jmath}(b) \in \operatorname{Span}_{\mathbf{A}}\left\{G\left(b^{\prime \prime}\right) \mid I^{\jmath}(b) \unlhd I^{\jmath}\left(b^{\prime}\right) \text { or }\left|I^{\jmath}\left(b^{\prime}\right)^{-}\right|<\left|I^{\jmath}(b)^{-}\right|\right\} .
$$

Hence, it suffices to show that $\left[e_{i} G^{\jmath}(b): G^{\jmath}\left(\widetilde{E}_{\underline{\underline{i}}} b\right)\right]=\left[\varphi_{\underline{i}}(b)+1\right]$. By the definitions of $e_{i}$ and $G^{\jmath}(b), e_{i} G^{\jmath}(b)$ is the sum of $E_{i} G(b)$ and a linear combination of weight vectors of $M$ of weight lower than wt $(b)+\alpha_{\underline{i}}$. We know from Proposition 9.1.1 (4) that 
$\left[E_{\underline{i}} G^{\jmath}(b): G\left(\widetilde{E}_{\underline{i}} b\right)\right]=\left[\varphi_{\underline{i}}(b)+1\right]$. Hence, we have $\left[e_{i} G^{\jmath}(b): G^{\jmath}\left(\widetilde{E}_{\underline{i}} b\right)\right]=\left[\varphi_{\underline{i}}(b)+1\right]$. This proves the assertion.

9.3. Global $\jmath$-crystal bases. Let $M \in \mathcal{O}_{\text {int }}^{\jmath},(\mathcal{L}, \mathcal{B})$ a $\jmath$-crystal basis of $M, \psi_{M}^{\jmath}$ a $\psi^{\jmath}$-involution, and $M_{\mathbf{A}}$ a $\mathbf{U}_{\mathbf{A}}^{\jmath}$-submodule of $M$. Suppose that $M$ has a $\jmath$-global basis $G^{\jmath}(\mathcal{B})$ with the associated balanced triple $\left(\mathcal{L}, M_{\mathbf{A}}, \psi_{M}^{\jmath}(\mathcal{L})\right)$.

Following K02, let us introduce modified Kashiwara operators:

Definition 9.3.1. For $n \in \mathbb{Z}$, set

$$
\begin{aligned}
\widetilde{f}_{i}^{(n)} & :=\sum_{t \geq 0,-n} f_{i}^{(n+t)} e_{i}^{(t)} A_{n}\left(t ; k_{i}\right), \\
\widetilde{f}_{1}^{(n)} & :=\sum_{t \geq 0,-n} f_{1}^{(n+t)} e_{1}^{(t)} a_{n}\left(t ; k_{1}\right),
\end{aligned}
$$

where

$$
\begin{aligned}
& A_{n}(t ; x):=(-1)^{t} q^{t(1-n)} x^{t} \prod_{s=0}^{t-1}\left(1-q^{n+2 s}\right), \\
& a_{n}(t ; x):=(-1)^{t} p^{t} q^{t(1-n)} x^{t} \prod_{s=0}^{t-1} q^{s}\left(1-q^{n+2 s}\right) .
\end{aligned}
$$

Lemma 9.3.2. Let $M \in \mathcal{O}_{\text {int }}^{\text {J }}$ with the -crystal basis $(\mathcal{L}, \mathcal{B})$. For $n \in \mathbb{Z}$, we have $\widetilde{f}_{i}^{(n)} \mathcal{L} \subset \mathcal{L}$, and $\widetilde{f}_{i}^{(n)} \mathcal{L}=\widetilde{f}_{i}^{n} \mathcal{L}$ modulo $q \mathcal{L}$.

Proof. If $i \neq 1$, then the statement follows from [K02, Proposition 6.1]. Hence, we prove the case when $i=1$. It suffices to prove the following: For each $u \in \mathcal{L}$ such that $e_{1} u=0, k_{1} u=q^{a} u, e_{1} f_{1} u=[b]\{a-b-1\} u$ with $a \in \mathbb{Z}$ and $b \in \mathbb{Z}_{\geq 0}$, we have $\widetilde{f}_{1}^{(n)} f_{1}^{(m)} u=c f_{1}^{(m+n)}$ for some $c \in 1+q \mathbf{A}_{0} \cap \mathbf{A}$. First of all, we have

$$
\widetilde{f}_{1}^{(n)} f_{1}^{(m)} u=\sum_{t \geq 0,-n} a_{n}\left(t ; q^{a-3 m}\right)\left[\begin{array}{c}
m+n \\
m-t
\end{array}\right]\left[\begin{array}{c}
b-m+t \\
t
\end{array}\right] \prod_{s=0}^{t-1}\{a-b-m+s\} f_{1}^{(m+n)} u .
$$

We compute the coefficient, say $A$, of the right-hand side as follows.

$$
\begin{aligned}
A & =\sum_{t \geq 0,-n} A_{n}\left(t ; q^{b-2 m}\right)\left[\begin{array}{c}
m+n \\
m-t
\end{array}\right]\left[\begin{array}{c}
b-m+t \\
t
\end{array}\right] \prod_{s=0}^{t-1}\left(1+p^{2} q^{2(a-b-m+s)}\right) \\
& =\sum_{t \geq 0,-n} B_{t}+p^{2} \sum_{t \geq 0,-n} B_{t} g_{t},
\end{aligned}
$$

where $B_{t}:=A_{n}\left(t ; q^{b-2 m}\right)\left[\begin{array}{c}m+n \\ m-t\end{array}\right]\left[\begin{array}{c}b-m+t \\ t\end{array}\right]$, and $g_{t} \in \mathbb{Z}\left[p, q, q^{-1}\right]$ with

$$
\prod_{s=0}^{t-1}\left(1+p^{2} q^{2(a-b-m+s)}\right)=1+p^{2} g_{t} \text {. }
$$

By the proof of [K02, Proposition 6.1], we have $B_{t} \in 1+q \mathbb{Z}[q]$. Also, it is clear that $p^{2} \sum_{t \geq 0,-n} B_{t} g_{t} \in p^{2} \mathbb{Z}\left[p, q, q^{-1}\right]$. Thus, we have $\widetilde{f}_{1}^{(n)} f_{1}^{(m)} u \in \mathcal{L}$ and $\widetilde{f}_{1}^{(n)} f_{1}^{(m)} u=$ $f_{1}^{(m+n)} u=\tilde{f}_{1}^{n} f_{1}^{(m)} u$ modulo $q \mathcal{L}$. This proves the lemma.

Proposition 9.3.3. Let $i \in \mathbb{I}^{\jmath}, b \in \mathcal{B}$ and $m \in \mathbb{Z}_{\geq 0}$. Then, we have the following.

$$
\text { (1) } \sum_{n \geq m} f_{i}^{(n)} M_{\mathbf{A}}=\bigoplus_{\substack{b^{\prime} \in \mathcal{B} \\ \varepsilon_{i}\left(b^{\prime}\right) \geq m}} \mathbf{A} G^{\jmath}\left(b^{\prime}\right) .
$$


(2) $\sum_{n \geq m} e_{i}^{(n)} M_{\mathbf{A}}=\bigoplus_{\substack{b^{\prime} \in \mathcal{B} \\ \varphi_{i}\left(b^{\prime}\right) \geq m}} \mathbf{A} G^{\jmath}\left(b^{\prime}\right)$ if $i \neq 1$.

(3) $f_{i} G^{\jmath}(b)=\left[\varepsilon_{i}(b)+1\right] G^{\jmath}\left(\widetilde{f}_{i} b\right)+\sum_{\varepsilon_{i}\left(b^{\prime}\right)>\varepsilon_{i}(b)+1}^{b^{\prime} \in \mathcal{B}} \varphi_{b^{\prime}, b}^{(i)} G^{\jmath}\left(b^{\prime}\right)$ for some $\varphi_{b^{\prime}, b}^{(i)} \in$ $q^{2-\varepsilon_{i}\left(b^{\prime}\right)} \mathbb{Q}[q]$.

(4) $e_{i} G^{\jmath}(b)=\left[\varphi_{i}(b)+1\right] G^{\jmath}\left(\widetilde{e}_{i} b\right)+\sum_{\substack{\varphi_{i}\left(b^{\prime}\right)>\varphi_{i}(b)+1 \\ b^{\prime} \in \mathcal{B}}} \varepsilon_{b^{\prime}, b}^{(i)} G^{\jmath}\left(b^{\prime}\right)$ for some $\varepsilon_{b^{\prime}, b}^{(i)} \in$ $q^{2-\varphi_{i}\left(b^{\prime}\right)} \mathbb{Q}[q]$ if $i \neq 1$.

Proof. Since $\left(e_{i}, k_{i}, f_{i}\right), i \neq 1$ forms an $\mathfrak{s l}_{2}$-triple, most of the assertions follows from Proposition 9.1.1. What we have to prove are assertions (1) and (3) for $i=1$. First, we prove part (1) by induction on $m$. When $m=0$, the both sides of the equation to be proved are 0. Assume that assertion (1) holds for all $m^{\prime}>m$. Let $b^{\prime} \in \mathcal{B}$ be such that $\varepsilon_{1}\left(b^{\prime}\right)=m$. Set $b_{0}^{\prime}:=\widetilde{e}_{1}^{m} b$, and consider $u:=\widetilde{f}_{1}^{(m)} G^{\jmath}\left(b_{0}^{\prime}\right)$. By the definition of $\widetilde{f}_{1}^{(m)}$ and Lemma 9.3.2, we have

$$
u-f_{1}^{(m)} G^{\jmath}\left(b_{0}^{\prime}\right) \in \sum_{n>m} f_{1}^{(n)} M_{\mathbf{A}} \text { and } u+q \mathcal{L}=b^{\prime} .
$$

By our inductive hypothesis, we can write

$$
u-f_{1}^{(m)} G^{J}\left(b_{0}^{\prime}\right)=\sum_{\substack{b^{\prime \prime} \in \mathcal{B} \\ \varepsilon_{1}\left(b^{\prime \prime}\right)>m}} a_{b^{\prime \prime}} G^{J}\left(b^{\prime \prime}\right)
$$

for some $a_{b^{\prime \prime}} \in \mathbf{A}$. Then, we can take $a_{b^{\prime \prime}}^{\prime} \in q \mathbb{Q}[q]$ in a way such that $a_{b^{\prime \prime}}-\overline{a_{b^{\prime \prime}}}=$ $a_{b^{\prime \prime}}^{\prime}-\overline{a_{b^{\prime \prime \prime}}^{\prime \prime}}$. Set $v:=u-\sum_{b^{\prime \prime}} a_{b^{\prime \prime}}^{\prime} G^{\jmath}\left(b^{\prime \prime}\right)=f_{1}^{(m)} G^{\jmath}\left(b_{0}^{\prime}\right)+\sum_{b^{\prime \prime}}\left(a_{b^{\prime \prime \prime}}-a_{b^{\prime \prime}}^{\prime}\right) G^{\jmath}\left(b^{\prime \prime}\right)$. Then, we have $v \in M_{\mathbf{A}} \cap \mathcal{L}, \psi_{M}^{3}(v)=v$, and $v+q \mathcal{L}=u+q \mathcal{L}=b^{\prime}$. These implies that $v=G^{\jmath}\left(b^{\prime}\right)$, and therefore, $G^{\jmath}\left(b^{\prime}\right) \in \sum_{n \geq m} f_{1}^{(n)} M_{\mathbf{A}}$. Hence, we obtain $\sum_{n \geq m} f_{i}^{(n)} M_{\mathbf{A}} \supset \bigoplus_{\substack{b^{\prime} \in \mathcal{B} \\ \varepsilon_{i}\left(b^{\prime}\right) \geq m}} \mathbf{A} G^{\jmath}\left(b^{\prime}\right)$.

We prove the opposite inclusion. For each $\lambda \in \Lambda^{J}$, we have

$$
\begin{aligned}
\left(M_{\mathbf{A}}\right)_{\lambda} & \subset \sum_{b \in \mathcal{B}_{\lambda}} \mathbf{A} G^{\jmath}(b) \\
& =\sum_{\substack{b \in \mathcal{B}_{\lambda} \\
\varepsilon_{1}(b)=0}} \mathbf{A} G^{\jmath}(b)+\sum_{\substack{b^{\prime} \in \mathcal{B}_{\lambda} \\
\varepsilon_{1}\left(b^{\prime}\right) \geq 1}} \mathbf{A} G^{\jmath}\left(b^{\prime}\right) \\
& \subset \sum_{\substack{b \in \mathcal{B}_{\lambda} \\
\varepsilon_{1}(b)=0}} \mathbf{A} G^{\jmath}(b)+\sum_{n \geq 1} f_{1}^{(n)}\left(M_{\mathbf{A}}\right)_{\lambda+n \gamma_{1}} .
\end{aligned}
$$

Hence, we obtain

$$
\begin{aligned}
f_{1}^{(m)}\left(M_{\mathbf{A}}\right)_{\lambda} & \subset \sum_{\substack{b \in \mathcal{B}_{\lambda} \\
\varepsilon_{1}(b)=0}} \mathbf{A} f_{1}^{(m)} G^{\jmath}(b)+\sum_{n \geq 1} f_{1}^{(m)} f_{1}^{(n)}\left(M_{\mathbf{A}}\right)_{\lambda+n \gamma_{1}} \\
& \subset \sum_{\substack{b \in \mathcal{B}_{\lambda} \\
\varepsilon_{1}(b)=0}} \mathbf{A} f_{1}^{(m)} G^{\jmath}(b)+\sum_{n \geq 1} f_{1}^{(m+n)}\left(M_{\mathbf{A}}\right)_{\lambda+n \gamma_{1}} \\
& =\sum_{\substack{b \in \mathcal{B}_{\lambda} \\
\varepsilon_{1}(b)=0}} \mathbf{A} f_{1}^{(m)} G^{\jmath}(b)+\sum_{\substack{b^{\prime} \in \mathcal{B}_{\lambda} \\
\varepsilon_{1}\left(b^{\prime}\right)>m}} \mathbf{A} G^{\jmath}\left(b^{\prime}\right) \quad \text { (by induction hypothesis). }
\end{aligned}
$$


Also, by the argument above, $f_{1}^{(m)} G^{\jmath}(b)$ with $\varepsilon_{1}(b)=0$ is contained in

$$
\sum_{\varepsilon\left(b^{\prime}\right) \geq m} \mathbf{A} G^{J}\left(b^{\prime}\right) \text {. }
$$

This completes the proof of part (1).

Next, we turn to prove assertion (3) for $i=1$ by descending induction on $m:=$ $\varepsilon_{1}(b)$. When $m$ is maximum among $\left\{\varepsilon_{1}\left(b^{\prime}\right) \mid b^{\prime} \in \mathcal{B}\right\}$, we have by (1) that

$$
f_{1} G^{\jmath}(b) \in \sum_{n>m} f_{1}^{(n)} M_{\mathbf{A}}=\sum_{\varepsilon_{1}\left(b^{\prime}\right)>m} \mathbf{A} G^{\jmath}\left(b^{\prime}\right)=0,
$$

and the equation in (3) holds. Assume that (3) is true for all $m^{\prime}>m$. As in the proof of (1), let us write

$$
\begin{gathered}
G^{\jmath}(b)=f_{1}^{(m)} G^{\jmath}\left(\widetilde{e}_{1}^{m} b\right)+\sum_{\substack{b^{\prime} \in \mathcal{B} \\
\varepsilon_{1}\left(b^{\prime}\right)>m}} c_{b^{\prime}} G^{\jmath}\left(b^{\prime}\right), \\
G^{\jmath}\left(\widetilde{f}_{1} b\right)=f_{1}^{(m+1)} G^{\jmath}\left(\widetilde{e}_{1}^{m} b\right)+\sum_{\substack{b^{\prime \prime} \in \mathcal{B} \\
\varepsilon_{1}\left(b^{\prime \prime}\right)>m+1}} d_{b^{\prime \prime}} G^{\jmath}\left(b^{\prime \prime}\right)
\end{gathered}
$$

for some $c_{b^{\prime}}, d_{b^{\prime \prime}} \in \mathbf{A}$. Then, we have

$$
\begin{aligned}
f_{1} G^{\jmath}(b)= & {[m+1] f_{1}^{(m+1)} G^{\jmath}\left(\widetilde{e}_{1}^{m} b\right)+\sum_{\varepsilon_{1}\left(b^{\prime}\right)>m} c_{b^{\prime}} f_{1} G^{\jmath}\left(b^{\prime}\right) } \\
= & {[m+1] f_{1}^{(m+1)} G^{\jmath}\left(\widetilde{e}_{1}^{m} b\right)+\sum_{\varepsilon_{1}\left(b^{\prime}\right)>m} c_{b^{\prime}}\left(\left[\varepsilon_{1}\left(b^{\prime}\right)+1\right] G^{\jmath}\left(\widetilde{f}_{1} b^{\prime}\right)\right.} \\
& \left.+\sum_{\varepsilon_{1}\left(b^{\prime \prime}\right)>\varepsilon_{1}\left(b^{\prime}\right)+1} \varphi_{b^{\prime \prime}, b^{\prime}}^{(1)} G^{\jmath}\left(b^{\prime \prime}\right)\right) \\
= & {[m+1] G^{\jmath}\left(\widetilde{f}_{1} b\right)+\sum_{\varepsilon_{1}\left(b^{\prime}\right)>m} c_{b^{\prime}}\left(\left[\varepsilon_{1}\left(b^{\prime}\right)+1\right] G^{\jmath}\left(\widetilde{f}_{1} b^{\prime}\right)\right.} \\
& \left.+\sum_{\varepsilon_{1}\left(b^{\prime \prime}\right)>\varepsilon_{1}\left(b^{\prime}\right)+1} \varphi_{b^{\prime \prime}, b^{\prime}}^{(1)} G^{\jmath}\left(b^{\prime \prime}\right)\right) \\
& -\sum_{\substack{b^{\prime \prime} \in \mathcal{B} \\
\varepsilon_{1}\left(b^{\prime \prime}\right)>m+1}}[m+1] d_{b^{\prime \prime}} G^{\jmath}\left(b^{\prime \prime}\right) .
\end{aligned}
$$

Thus, we obtain that $f_{i} G^{\jmath}(b)=\left[\varepsilon_{i}(b)+1\right] G^{\jmath}\left(\widetilde{f}_{i} b\right)+\sum_{\varepsilon_{i}\left(b^{\prime}\right)>\varepsilon_{i}(b)+1} \varphi_{b^{\prime}, b}^{(i)} G^{\jmath}\left(b^{\prime}\right)$ for some $\varphi_{b^{\prime}, b}^{(i)} \in \mathbf{A}$. It remains to prove that $\varphi_{b^{\prime}, b}^{(i)} \in q^{2-\varepsilon_{1}\left(b^{\prime}\right)} \mathbb{Q}[q]$. Let us write

$$
G^{\jmath}(b)=\sum_{k \geq m} f_{1}^{(k)} u_{k}
$$

for some $u_{k} \in \mathcal{L}_{\mathrm{wt}^{\jmath}(b)+k \gamma_{1}}$ such that $e_{1} u_{k}=0$. Note that $G^{\jmath}(b)+q \mathcal{L}=u_{m}+q \mathcal{L}$. Then, we have

$$
f_{1} G^{\jmath}(b)=[m+1] f_{1}^{(m+1)} u_{m}+\sum_{k>m}[k+1] f_{1}^{(k+1)} u_{k}
$$

and that $f_{1}^{(m+1)} u_{m} \in \mathcal{L}, f_{1}^{(m+1)} u_{m}+q \mathcal{L}=\tilde{f}_{1} b$. Hence, we have $f_{1} G^{\jmath}(b)=[m+$ 1] $G^{\jmath}\left(\widetilde{f}_{1} b\right)+\sum_{k>m}[k+1] f_{1}^{(k+1)} u_{k}$ modulo $q^{2-m} \mathcal{L}$. Then, rewriting $f_{1}^{(k+1)} u_{k}$ as a sum 
of $G^{\jmath}\left(b^{\prime}\right), \varepsilon_{1}\left(b^{\prime}\right) \leq k+1$ with coefficients in $q \mathbf{A}_{0}$, we conclude that the coefficient of $G^{\jmath}\left(b^{\prime}\right)$ in $f_{1} G^{\jmath}(b)$ lies in $q^{2-\varepsilon_{1}\left(b^{\prime}\right)} \mathbf{A}_{0} \cap \mathbf{A}=q^{2-\varepsilon_{1}\left(b^{\prime}\right)} \mathbb{Q}[q]$. This completes the proof.

For a bipartition $\boldsymbol{\lambda} \in P^{\jmath}(M)$, define $I_{\boldsymbol{\lambda}}(M), W_{\succeq \boldsymbol{\lambda}}(M), W_{\succ \boldsymbol{\lambda}}(M)$, and $W_{\boldsymbol{\lambda}}(M)$ in a similar way as $I_{\lambda}, W_{\succeq \lambda}, W_{\succ \lambda}$, and $W_{\lambda}$, respectively.

Definition 9.3.4. We say that $M$ has the property $(*)$ if there exists a poset $(S, \leq)$ and a map $s: \mathcal{B} \rightarrow S$ satisfying the following:

(1) The abelian group $Q:=\sum_{i \in \mathbb{I}} \mathbb{Z} \alpha_{i}$ acts on $S$ freely; the action is written additively.

(2) $\sigma \leq \sigma+\lambda$ for all $\lambda \in Q_{+}, \sigma \in S$.

(3) $\sigma+\lambda \leq \sigma^{\prime}+\lambda$ for all $\lambda \in Q, \sigma \leq \sigma^{\prime} \in S$.

(4) $s(b)=s\left(b^{\prime}\right)$ only if $\operatorname{wt}(b)=\operatorname{wt}\left(b^{\prime}\right)$ for all $b, b^{\prime} \in \mathcal{B}$.

(5) For $b \in \mathcal{B}$ and $i \in \mathbb{I}^{\jmath} \backslash\{1\}, s\left(\widetilde{E}_{\underline{i}} b\right)=s(b)+\alpha_{\underline{i}}$ if $\widetilde{E}_{\underline{\underline{i}}} b \neq 0$.

(6) For $i \in \mathbb{I}^{\jmath} \backslash\{1\}$,

$$
\begin{aligned}
& e_{i} G^{\jmath}(b)=\left[\varphi_{\underline{i}}(b)+1\right] G^{\jmath}\left(\widetilde{E}_{\underline{i}} b\right)+\sum_{\substack{b^{\prime} \in \mathcal{B} \backslash\left\{\widetilde{E}_{\underline{E}} b\right\} \\
\mathrm{wt}^{\jmath}\left(b^{\prime}\right)=\mathrm{wt}^{\jmath}(b)+\gamma_{i} \text { and } s\left(b^{\prime}\right) \leq s(b)+\alpha_{i}}} e_{b^{\prime}, b}^{(i)} G^{\jmath}\left(b^{\prime}\right), \\
& f_{i} G^{\jmath}(b)=\left[\varphi_{-\underline{i}}(b)+1\right] G^{\jmath}\left(\widetilde{E}_{-\underline{i}} b\right)+\sum_{\substack{b^{\prime} \in \mathcal{B} \backslash\left\{\widetilde{E}_{-\underline{i}} b\right\} \\
\mathrm{wt}^{\jmath}\left(b^{\prime}\right)=\mathrm{wt}^{\jmath}(b)-\gamma_{i} \text { and } s\left(b^{\prime}\right) \leq s(b)+\alpha_{-\underline{i}}}} f_{b^{\prime}, b}^{(i)} G^{\jmath}\left(b^{\prime}\right)
\end{aligned}
$$

for some $e_{b^{\prime}, b}^{(i)}, f_{b^{\prime}, b}^{(i)} \in \mathbf{A}$.

Lemma 9.3.5. Let $M \in \mathcal{O}_{\text {int }}^{\jmath}$, and $\mathcal{L}, \mathcal{B}, \psi_{M}^{J}, M_{\mathbf{A}}$ as above.

(1) If $r=1$, then $M$ has the property $(*)$.

(2) If $M \in \mathcal{O}_{\text {int }}$ and the global J-crystal basis is the $\mathrm{J}$-canonical basis, then $M$ has the property $(*)$.

Proof. Setting $S$ and $s$ to be $\Lambda$ and wt, respectively, part (1) is obvious, and part (2) follows from Proposition 9.2.2

The main result in this paper is the following:

Theorem 9.3.6. Suppose that $M$ has the property (*). Then, for each $\boldsymbol{\lambda} \in P^{J}(M)$, the following hold:

(1) $W_{\succeq \boldsymbol{\lambda}}(M)$ has a global j-crystal basis $W_{\succeq \boldsymbol{\lambda}}\left(G^{\jmath}(\mathcal{B})\right):=\left\{G^{\jmath}(b) \mid I(b) \succeq\right.$ $\boldsymbol{\lambda}\}$ with the associated balanced triple $\left(W_{\succeq \boldsymbol{\lambda}}(\mathcal{L}), W_{\succeq \boldsymbol{\lambda}}\left(M_{\mathbf{A}}\right), W_{\succeq \boldsymbol{\lambda}}\left(\psi_{M}^{\jmath}(\mathcal{L})\right)\right)$, where $W_{\succeq \boldsymbol{\lambda}}(\mathcal{L}):=W_{\succ \boldsymbol{\lambda}}(M) \cap \mathcal{L}$, and so on.

(2) $W_{\succ \boldsymbol{\lambda}}(M)$ has a global j-crystal basis $W_{\succ \boldsymbol{\lambda}}\left(G^{\jmath}(\mathcal{B})\right):=\left\{G^{\jmath}(b) \mid I(b) \succ\right.$ $\lambda\}$ with the associated balanced triple $\left(W_{\succ \boldsymbol{\lambda}}(\mathcal{L}), W_{\succ \boldsymbol{\lambda}}\left(M_{\mathbf{A}}\right), W_{\succ \boldsymbol{\lambda}}\left(\psi_{M}^{J}(\mathcal{L})\right)\right)$, where $W_{\succ \boldsymbol{\lambda}}(\mathcal{L}):=W_{\succ \boldsymbol{\lambda}}(M) \cap \mathcal{L}$, and so on.

(3) $W_{\boldsymbol{\lambda}}(M)$ has a global $\}$-crystal basis $W_{\boldsymbol{\lambda}}\left(G^{\jmath}(\mathcal{B})\right):=\left\{G^{\jmath}(b)+W_{\succ \boldsymbol{\lambda}}(M) \mid I(b)=\right.$ $\lambda\}$ with the associated balanced triple $\left(W_{\boldsymbol{\lambda}}(\mathcal{L}), W_{\boldsymbol{\lambda}}\left(M_{\mathbf{A}}\right), W_{\boldsymbol{\lambda}}\left(\psi_{M}^{3}(\mathcal{L})\right)\right)$, where $W_{\boldsymbol{\lambda}}(\mathcal{L}):=W_{\succeq \boldsymbol{\lambda}}(\mathcal{L}) / W_{\succ \boldsymbol{\lambda}}(\mathcal{L})$, and so on. 
(4) There exists a $\mathbf{U}^{3}$-module isomorphism $\xi: L(\boldsymbol{\lambda})^{\oplus m_{\boldsymbol{\lambda}}} \rightarrow W_{\boldsymbol{\lambda}}(M)$ which induces an isomorphism

$$
\begin{aligned}
& \left(\mathcal{L}(\boldsymbol{\lambda})^{\oplus m_{\boldsymbol{\lambda}}},\left(L(\boldsymbol{\lambda})_{\mathbf{A}}\right)^{\oplus m_{\boldsymbol{\lambda}}}, \psi_{\boldsymbol{\lambda}}^{J}(\mathcal{L}(\boldsymbol{\lambda}))^{\oplus m_{\boldsymbol{\lambda}}}\right) \simeq\left(W_{\boldsymbol{\lambda}}(\mathcal{L}), W_{\boldsymbol{\lambda}}\left(M_{\mathbf{A}}\right), W_{\boldsymbol{\lambda}}\left(\psi_{M}^{J}(\mathcal{L})\right)\right), \\
& \text { where } m_{\boldsymbol{\lambda}}:=\operatorname{dim} \operatorname{Hom}_{\mathbf{U}^{\jmath}}(L(\boldsymbol{\lambda}), M) \text { denotes the multiplicity of } L(\boldsymbol{\lambda}) \text { in } M \text {. }
\end{aligned}
$$

The proof will be given in Section 10.

Corollary 9.3.7. Let $\boldsymbol{\lambda} \in P^{\jmath}$. Then, $G_{\text {low }}^{\jmath}(\boldsymbol{\lambda})$ is a unique global $\jmath$-crystal basis of $L(\boldsymbol{\lambda})$ satisfying the property $(*)$.

9.4. Operators $\tilde{e}_{i^{+}}$and $\tilde{f}_{i^{+}}$. The definitions of $\tilde{e}_{i^{\prime}}$ and $\tilde{f}_{i^{\prime}}$ given in W17] are artificial, namely, they are defined by means of a distinguished basis $G_{\text {low }}^{\text {J }}(\boldsymbol{\lambda}), \boldsymbol{\lambda} \in$ $P^{J}$ (in W17, it is denoted by $\left\{b_{T} \mid T \in \mathcal{B}(\boldsymbol{\lambda})\right\}$ ). Here, we define new operators $\widetilde{e}_{i^{+}}$ and $\widetilde{f}_{i^{+}}$for $i \in \mathbb{I}^{\jmath} \backslash\{1\}$, and then, explain that the operators $\widetilde{e}_{i^{\prime}}$ and $\widetilde{f}_{i^{\prime}}$ on $\jmath$-crystal bases are in fact intrinsic.

Lemma 9.4.1. Let $r \geq 2, \lambda \in P^{\jmath}$, and consider the irreducible highest weight module $L(\boldsymbol{\lambda})$. As a $\mathbf{U}_{r-1}^{\jmath}$-module, $L(\boldsymbol{\lambda})$ is multiplicity-free.

Proof. Let $b \in \mathcal{B}(\boldsymbol{\lambda})$ be a $\mathbf{U}_{r-1}^{\jmath}$-highest weight vector with highest weight, say, $\boldsymbol{\mu} \in P_{r-1}^{\jmath}$. If we identify $\mathcal{B}(\boldsymbol{\lambda})$ with $\operatorname{SST}(\boldsymbol{\lambda})$, we have $T_{b}^{\jmath} \downarrow_{r-1}=T_{\boldsymbol{\mu}}$. Since the entries of the boxes of $T_{b^{\prime}}^{3}$ corresponding to $\boldsymbol{\lambda} / \boldsymbol{\mu}$ are either $-r$ or $r$, it must hold that $\boldsymbol{\lambda} / \boldsymbol{\mu}$ is a horizontal strip. Conversely, given $\boldsymbol{\mu} \in P_{r-1}^{J}$ such that $\boldsymbol{\lambda} / \boldsymbol{\mu}$ is a horizontal strip, there exists a unique $b \in \mathcal{B}(\boldsymbol{\lambda})$ which is a $\mathbf{U}_{r-1}^{\jmath}$-highest weight vector with highest weight $\boldsymbol{\mu}$. This proves the lemma.

Lemma 9.4.2. Let $r \geq 2, \boldsymbol{\lambda} \in P^{\jmath}$. Let $b \in \mathcal{B}(\boldsymbol{\lambda})$ be such that $\widetilde{e}_{r^{\prime}} b \neq 0$. Then, there exist unique $b^{\prime} \in \mathcal{B}(\boldsymbol{\lambda})$ and $j \in \mathbb{I}^{J} \backslash\{1\}$ satisfying the following:

- $b^{\prime}$ is a $\mathbf{U}_{r-1}^{\jmath}$-highest weight vector.

- There exist unique $\varepsilon_{i} \in\{\emptyset, \prime\}$ for each $j \leq i \leq r-1$ such that $b=$ $\widetilde{f}_{r^{\prime}} \widetilde{f}_{(r-1)^{\varepsilon_{r-1}}} \cdots \widetilde{f}_{j_{j}} b^{\prime}$.

Proof. By the definition of $\widetilde{e}_{r^{\prime}}, b$ is a $\mathbf{U}_{r-1}^{J}$-highest weight vector with highest weight, say, $\boldsymbol{\mu} \in P_{r-1}^{J}$ such that $\left(T_{b}^{J}\right)^{-}=T_{\boldsymbol{\lambda}}^{-}$. Then, $T_{\widetilde{e}_{r^{\prime}}}^{\jmath} \downarrow_{r-1}$ is obtained from $T_{\boldsymbol{\mu}}$ by adding a box $r-1$ to the $(j-1)$-th row for some uniquely determined $j \in$ $\mathbb{I}^{\jmath} \backslash\{1\}$. Set $b_{r-1}:=\widetilde{e}_{r^{\prime}} b$. Now, we have exactly one of the following; $\widetilde{e}_{r-1} b_{r-1} \neq 0$ or $\widetilde{e}_{(r-1)^{\prime}} b_{r-1} \neq 0$. Choose a unique $\varepsilon_{r-1} \in\{\emptyset, /\}$ in a way such that $b_{r-2}:=$ $\widetilde{e}_{(r-1)^{\varepsilon_{r-1}}} b_{r-1} \neq 0$. Then, $T_{b_{r-2}}^{j} \downarrow_{r-1}$ is obtained from $T_{\boldsymbol{\mu}}$ by adding a box $r-2$ to the $(j-1)$-th row. Repeating this procedure, we obtain $\varepsilon_{i} \in\{\emptyset, \prime\}$ and $b_{i-1} \in \mathcal{B}(\boldsymbol{\lambda})$ for $j \leq i \leq r-1$. By the construction, $T_{b_{j-1}}^{j} \downarrow_{r-1}$ is obtained from $T_{\boldsymbol{\mu}}$ by adding a box $j-1$ to the $(j-1)$-th row, which turned out to be $T_{\boldsymbol{\mu}^{\prime}}$, where $\boldsymbol{\mu}^{\prime} \in P_{r-1}^{J}$ such that $\boldsymbol{\mu}_{k}^{\prime}=\boldsymbol{\mu}_{k}+\delta_{k, j-1}, k \in\{-(r-1), \ldots, r-1\}$. Hence, $b_{j-1}$ is a $\mathbf{U}_{r-1^{-}}^{\jmath}$ highest weight vector, and we have $b=\widetilde{f}_{r^{\prime}} \widetilde{f}_{(r-1)^{\varepsilon_{r-1}}} \cdots \widetilde{f}_{j^{\varepsilon}} b_{j-1}$. This proves the assertion.

Set $E_{r}(\boldsymbol{\lambda}):=\left\{\boldsymbol{\mu} \in P_{r-1}^{J} \mid \boldsymbol{\mu}^{-}=\boldsymbol{\lambda}^{-} \downarrow_{r-1}\right.$ and $\boldsymbol{\lambda}^{+} / \boldsymbol{\mu}^{+}$is a horizontal strip $\}$. Then, the assignment

$$
\left\{b \in \mathcal{B}(\boldsymbol{\lambda}) \mid \widetilde{e}_{r^{\prime}} b \neq 0\right\} \rightarrow E_{r}(\boldsymbol{\lambda}) ; b \mapsto I_{r-1}^{\jmath}(b)
$$


is bijective. To each $\boldsymbol{\mu} \in E_{r}(\boldsymbol{\lambda})$, we associate $b, b^{\prime} \in \mathcal{B}(\boldsymbol{\lambda}), j \in \mathbb{I}^{\jmath} \backslash\{1\}$, and $\varepsilon_{i} \in\{\emptyset, \prime\}, j \leq i \leq r-1$ as in Lemma 9.4 .2

Let $r \geq 2$. We define operators $\widetilde{e}_{l^{+}}$and $\widetilde{f}_{l^{+}}$on every $\mathbf{U}^{\jmath}$-modules in $\mathcal{O}_{\text {int }}^{J}$ inductively for all $2 \leq l<r$. Let $\boldsymbol{\lambda} \in P^{\jmath}$. We define the linear operator $\widetilde{e}_{r}+$ on $L(\boldsymbol{\lambda})$ by

$$
\widetilde{e}_{r^{+}}:=\bigoplus_{\boldsymbol{\mu} \in E_{r}(\boldsymbol{\lambda})} p_{2}(\boldsymbol{\mu}) \circ \frac{1}{\left[\varphi_{\underline{r}}\left(b_{\boldsymbol{\mu}}\right)+1\right]} e_{r} \circ p_{1}(\boldsymbol{\mu}),
$$

where $b_{\boldsymbol{\mu}} \in \mathcal{B}(\boldsymbol{\lambda})$ is the corresponding element to $\boldsymbol{\mu} \in E_{r}(\boldsymbol{\lambda}), p_{1}(\boldsymbol{\mu})$ is the projection from $L(\boldsymbol{\lambda})$ to the one-dimensional subspace $L(\boldsymbol{\mu})_{\mathrm{wt}^{\jmath}(\boldsymbol{\mu})}$;

$$
L(\boldsymbol{\mu})_{\mathrm{wt}^{\jmath}(\boldsymbol{\mu})} \subset L(\boldsymbol{\mu}) \underset{\text { multiplicity free }}{\hookrightarrow} L(\boldsymbol{\lambda}),
$$

and $p_{2}(\boldsymbol{\mu})$ is the projection from $L(\boldsymbol{\lambda})$ to the one-dimensional subspace $\widetilde{f}_{(r-1)^{\delta_{r-1}}}$ $\cdots \widetilde{f}_{j^{\delta_{j}}} L\left(\boldsymbol{\mu}^{\prime}\right)_{\mathrm{wt}^{\jmath}\left(\boldsymbol{\mu}^{\prime}\right)}$;

$$
\tilde{f}_{(r-1)^{\delta_{r-1}}} \cdots \tilde{f}_{j^{\delta_{j}}} L\left(\boldsymbol{\mu}^{\prime}\right)_{\mathrm{wt}^{\jmath}\left(\boldsymbol{\mu}^{\prime}\right)} \subset L\left(\boldsymbol{\mu}^{\prime}\right) \underset{\text { multiplicity free }}{\hookrightarrow} L(\boldsymbol{\lambda}),
$$

where $\delta_{l}=\emptyset$ if $\varepsilon_{l}=\emptyset$, and $\delta_{l}=+$ if $\varepsilon_{l}=\prime$ for $l=j, \ldots, r-1$. Also, we define $\tilde{f}_{r^{+}}$ by

$$
\widetilde{f}_{r^{+}}=\bigoplus_{\boldsymbol{\mu} \in E_{r}(\boldsymbol{\lambda})} \widetilde{e}_{r^{+}}^{-1} \circ p_{2}(\boldsymbol{\mu})
$$

where $\widetilde{e}_{r^{+}}^{-1}$ is the inverse of the linear isomorphism $\widetilde{e}_{r^{+}}: L(\boldsymbol{\mu})_{\mathrm{wt}^{\jmath}(\boldsymbol{\mu})} \rightarrow \widetilde{f}_{(r-1)^{\delta_{r-1}}}$ $\cdots \widetilde{f}_{j^{\delta_{j}}} L\left(\boldsymbol{\mu}^{\prime}\right)_{\mathrm{wt}^{\jmath}\left(\boldsymbol{\mu}^{\prime}\right)}$. Finally, we extend the definitions of $\widetilde{e}_{r^{+}}$and $\widetilde{f}_{r^{+}}$to a general $\mathrm{U}^{\jmath}$-module $M \in \mathcal{O}_{\text {int }}^{\text {J }}$ by the complete reducibility of $M$.

Proposition 9.4.3. Let $\boldsymbol{\lambda} \in P^{J}$ and $v \in L(\boldsymbol{\lambda})$ a highest weight vector. Then, we have

$$
\begin{aligned}
& \mathcal{L}(\boldsymbol{\lambda})=\operatorname{Span}_{\mathbf{A}_{0}}\left\{\widetilde{f}_{i_{1}} \cdots \widetilde{f}_{i, l} v \mid l \in \mathbb{Z}_{\geq 0}, i_{1}, \ldots, i_{l} \in \mathbb{I}^{\jmath} \sqcup\left\{2^{+}, \ldots, r^{+}\right\}\right\}, \\
& \mathcal{B}(\boldsymbol{\lambda})=\left\{\widetilde{f}_{i_{1}} \cdots \widetilde{f}_{i, l} v+q \mathcal{L}(\boldsymbol{\lambda}) \mid l \in \mathbb{Z}_{\geq 0}, i_{1}, \ldots, i_{l} \in \mathbb{I}^{\jmath} \sqcup\left\{2^{+}, \ldots, r^{+}\right\}\right\} \backslash\{0\} .
\end{aligned}
$$

Moreover, on $\mathcal{B}(\boldsymbol{\lambda})$, we have $\widetilde{e}_{i^{\prime}}=\widetilde{e}_{i^{+}}$and $\widetilde{f}_{i^{\prime}}=\widetilde{f}_{i^{+}}$for all $i \in \mathbb{I}^{\jmath} \backslash\{1\}$.

Proof. We proceed by induction on $r$. Assume that the assertion holds for all $2 \leq l<r$ (we assume nothing when $r=2$ ). Let $\boldsymbol{\mu} \in E_{r}(\boldsymbol{\lambda})$ and $b_{\boldsymbol{\mu}}, b^{\prime}, \boldsymbol{\mu}^{\prime}$ be as above. By the uniqueness of the $\jmath$-crystal bases for $\mathbf{U}_{r-1}^{J}$-modules, there exists a unique $v_{\boldsymbol{\mu}} \in \mathcal{L}(\boldsymbol{\lambda})$ such that $\mathbf{U}_{r-1}^{J} v_{\boldsymbol{\mu}}=L(\boldsymbol{\mu}), v_{\boldsymbol{\mu}}+q \mathcal{L}(\boldsymbol{\lambda})=b_{\boldsymbol{\mu}}$. Then, we can write

$$
v_{\boldsymbol{\mu}}=G_{\text {low }}^{J}\left(b_{\boldsymbol{\mu}}\right)+\sum_{b^{\prime} \in \mathcal{B}(\boldsymbol{\lambda}) \backslash\left\{b_{\boldsymbol{\mu}}\right\}} a_{b^{\prime}} G_{\text {low }}^{\text {low }}\left(b^{\prime}\right)
$$

for some $a_{b^{\prime}} \in q \mathbf{A}_{0}$. Note that this equation implies that $\widetilde{e}_{r^{\prime}}\left(v_{\boldsymbol{\mu}}\right) \in G_{\text {low }}^{J}\left(\widetilde{e}_{r^{\prime}} b_{\boldsymbol{\mu}}\right)+$ $q \mathcal{L}(\boldsymbol{\lambda})$. Also, we have

$$
\frac{1}{\left[\varphi_{\underline{r}}\left(b_{\boldsymbol{\mu}}\right)+1\right]} e_{r} v_{\boldsymbol{\mu}}=G_{\text {low }}^{J}\left(\widetilde{e}_{r^{\prime}} b_{\boldsymbol{\mu}}\right)+\sum_{b^{\prime} \in \mathcal{B}(\boldsymbol{\lambda})} c_{b^{\prime}} G_{\text {low }}^{J}\left(b^{\prime}\right) \quad\left(\text { since } \widetilde{e}_{r^{\prime}} b_{\boldsymbol{\mu}}=\widetilde{E}_{\underline{\underline{r}}} b_{\boldsymbol{\mu}} .\right)
$$

for some $c_{b^{\prime}} \in \mathbf{A}$. Again, by the complete reducibility of the $\mathbf{U}_{r-1}^{J}$-crystal bases, there exists a unique $v_{\boldsymbol{\mu}^{\prime}} \in \mathcal{L}(\boldsymbol{\lambda})$ such that $\mathbf{U}_{r-1}^{\jmath} v_{\boldsymbol{\mu}^{\prime}}=L\left(\boldsymbol{\mu}^{\prime}\right), v_{\boldsymbol{\mu}^{\prime}}+q \mathcal{L}(\boldsymbol{\lambda})=b^{\prime}$. By 
our induction hypothesis, we have $u:=\tilde{f}_{(r-1)^{\delta_{r-1}}} \cdots \tilde{f}_{j^{\delta_{j}}}\left(v_{\boldsymbol{\mu}^{\prime}}\right) \in \mathcal{L}(\boldsymbol{\lambda}) \cap \mathbf{U}_{r-1}^{\jmath} v_{\boldsymbol{\mu}^{\prime}}$ and $u+q \mathcal{L}(\boldsymbol{\lambda})=\widetilde{e}_{r^{\prime}} b_{\boldsymbol{\mu}}$. Then, we can write

$$
u=G_{\text {low }}^{J}\left(\widetilde{e}_{r^{\prime}} b_{\boldsymbol{\mu}}\right)+\sum_{b^{\prime} \in \mathcal{B}(\boldsymbol{\lambda})} d_{b^{\prime}} G_{\text {low }}^{J}\left(b^{\prime}\right)
$$

for some $d_{b^{\prime}} \in q \mathbf{A}_{0}$. Hence, we have

$$
\widetilde{e}_{r+}\left(v_{\boldsymbol{\mu}}\right) \in G_{\text {low }}^{J}\left(\widetilde{e}_{r^{\prime}} b_{\boldsymbol{\mu}}\right)+q \mathcal{L}(\boldsymbol{\lambda}) .
$$

Since we took $\boldsymbol{\mu} \in E_{r}(\boldsymbol{\lambda})$ arbitrarily, this equation ensures that $\widetilde{e}_{r^{+}}$preserves $\mathcal{L}(\boldsymbol{\lambda})$ and $\mathcal{B}(\boldsymbol{\lambda}) \sqcup\{0\}$, and that $\widetilde{e}_{r^{+}}=\widetilde{e}_{r^{\prime}}$ on $\mathcal{B}(\boldsymbol{\lambda})$. By the definition of $\widetilde{f}_{r^{+}}$, it also preserves $\mathcal{L}(\boldsymbol{\lambda})$ and $\mathcal{B}(\boldsymbol{\lambda}) \sqcup\{0\}$, and coincides with $\widetilde{f}_{r^{\prime}}$ on $\mathcal{B}(\boldsymbol{\lambda})$. Now, the assertions are clear by the definition of $(\mathcal{L}(\boldsymbol{\lambda}), \mathcal{B}(\boldsymbol{\lambda}))$.

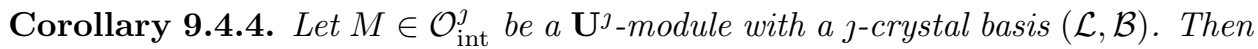
$\widetilde{e}_{i^{\prime}}=\widetilde{e}_{i^{+}}$and $\widetilde{f}_{i^{\prime}}=\widetilde{f}_{i^{+}}$on $\mathcal{B}$ for all $i \in \mathbb{I}^{\jmath} \backslash\{1\}$.

\section{Proof of Theorem 9.3.6}

For a $\mathbf{U}^{\jmath}$-module $M$ with a global $\jmath$-crystal basis $G^{\jmath}(\mathcal{B})$, and for $m \in M, b \in \mathcal{B}$, let $\left[m: G^{\jmath}(b)\right]$ denote the coefficient of $G^{\jmath}(b)$ in $m$.

10.1. The $r=1$ case. In this subsection, we prove Theorem 9.3.6 for $r=1$.

Proof of Theorem 9.3.6. We proceed by descending induction on $\boldsymbol{\lambda}$ with respect to $\preceq$. Assume that the statement holds for all $\boldsymbol{\lambda}^{\prime} \succ \boldsymbol{\lambda}$. Replacing $M$ with $M / W_{\succ \boldsymbol{\lambda}}(M)$, we may assume that $\boldsymbol{\lambda}$ is maximal among $P^{\jmath}(M)$. Let $b_{1}, \ldots, b_{m_{\boldsymbol{\lambda}}} \in \mathcal{B}$ and $u_{1}, \ldots, u_{m_{\boldsymbol{\lambda}}} \in \mathcal{L}$ be distinct highest weight vectors of type $\boldsymbol{\lambda}$ with $u_{i}+q \mathcal{L}=b_{i}$, $i=1, \ldots, m_{\boldsymbol{\lambda}}$. By retaking the $u_{i}$ 's if necessary, we may assume that $\left[u_{i}: G^{\jmath}\left(b_{j}\right)\right]=$ $\delta_{i, j}$ for all $i, j$. Fix $i$ arbitrarily, and set $b:=b_{i}, u:=u_{i}$. Then, we can write

$$
u=G^{\jmath}(b)+\sum_{\substack{b^{\prime} \\ I^{\jmath}\left(b^{\prime}\right) \nsucceq \boldsymbol{\lambda}}} c_{b^{\prime}} G^{\jmath}\left(b^{\prime}\right), \quad c_{b^{\prime}} \in q \mathbf{A}_{0} .
$$

We first prove that $c_{b^{\prime}}=0$ for all $b^{\prime}$ with $\varepsilon_{1}\left(b^{\prime}\right)=0$. Assume contrary, and take $b^{\prime} \in \mathcal{B} \backslash\{b\}$ such that $c_{b^{\prime}} \neq 0, \varepsilon_{1}\left(b^{\prime}\right)=0$, and $\varphi_{1}\left(b^{\prime}\right)$ is minimal among $\left\{\varphi_{1}\left(b^{\prime \prime}\right) \mid c_{b^{\prime \prime}} \neq 0, \varepsilon_{1}\left(b^{\prime \prime}\right)=0\right\}$. Set $\boldsymbol{\mu}:=I^{J}\left(b^{\prime}\right)$. Then, we have $\operatorname{wt}^{\jmath}(\boldsymbol{\mu})=\mathrm{wt}^{\jmath}(\boldsymbol{\lambda})$, in particular, $\boldsymbol{\mu}_{0}=\boldsymbol{\lambda}_{0}$. Since $\boldsymbol{\mu} \nsucceq \boldsymbol{\lambda}$, we have $\varphi_{1}\left(b^{\prime}\right)=\boldsymbol{\mu}_{0}-\boldsymbol{\mu}_{-1}>\boldsymbol{\lambda}_{0}-\boldsymbol{\lambda}_{-1}=\varphi_{1}(b)$. Hence, we have

$$
\begin{aligned}
& -f_{1}^{\left(\varphi_{1}(b)+1\right)} G^{\jmath}(b)=c_{b^{\prime}}\left(G^{\jmath}\left(\tilde{f}_{1}^{\varphi_{1}(b)+1} b^{\prime}\right)+\sum_{\varepsilon_{1}\left(b^{\prime \prime}\right)>\varphi_{1}(b)+1} d_{b^{\prime \prime}, b^{\prime}} G^{\jmath}\left(b^{\prime \prime}\right)\right) \\
& +\sum_{b^{\prime \prime \prime} \neq b^{\prime}} \sum_{\substack{b_{1}^{\prime \prime \prime \prime} \\
\varepsilon_{1}\left(b^{\prime \prime \prime \prime}\right) \geq \varepsilon_{1}\left(b^{\prime \prime \prime}\right)+\varphi_{1}(b)+1}} d_{b^{\prime \prime \prime \prime}, b^{\prime \prime \prime}} G^{J}\left(b^{\prime \prime \prime \prime}\right),
\end{aligned}
$$

for some $d_{b_{1}, b_{2}} \in \mathbf{A}$. By our assumption, the coefficient of $G^{\jmath}\left(\tilde{f}_{1}^{\varphi_{1}(b)+1} b^{\prime}\right)$ in the right-hand side is equal to $c_{b^{\prime}}$. On the other hand, the left-hand side is fixed by $\psi_{M}^{J}$, and it belongs to $M_{\mathbf{A}}$. Therefore, we have $c_{b^{\prime}} \in q \mathbf{A}_{0} \cap \mathbf{A}$ and $\overline{c_{b^{\prime}}}=c_{b^{\prime}}$, which implies $c_{b^{\prime}}=0$.

Next, we prove that $c_{b^{\prime}}=0$ for all $b^{\prime}$ with $\varepsilon_{1}\left(b^{\prime}\right)>0$. Assume contrary that $c_{b^{\prime}} \neq 0$ for some such $b^{\prime}$. Set $\boldsymbol{\mu}:=I^{\jmath}\left(b^{\prime}\right)$. Since $\boldsymbol{\lambda}$ is maximal, we have $\boldsymbol{\mu}_{0}+\boldsymbol{\mu}_{-1}<$ 
$\boldsymbol{\lambda}_{0}+\boldsymbol{\lambda}_{-1}$. Substituting $\boldsymbol{\mu}_{0}=\boldsymbol{\lambda}_{0}+\varepsilon_{1}\left(b^{\prime}\right), \boldsymbol{\mu}_{-1}=\boldsymbol{\lambda}_{0}-\varphi_{1}\left(b^{\prime}\right)$, and $\boldsymbol{\lambda}_{0}-\boldsymbol{\lambda}_{-1}=$ $\varphi_{1}(b)$, we obtain $\varphi_{1}\left(b^{\prime}\right)>\varphi_{1}(b)+\varepsilon_{1}\left(b^{\prime}\right)$. We may assume that $\left(\varepsilon_{1}\left(b^{\prime}\right), \varphi_{1}\left(b^{\prime}\right)\right)$ is minimal (with respect to the lexigographical order) among such $b^{\prime}$ 's. Then, for all $t=1, \ldots, \varepsilon_{1}\left(b^{\prime}\right)+1$, we have

$$
-f_{1}^{\left(\varphi_{1}(b)+t\right)} G^{\jmath}(b)=c_{b^{\prime}}\left[\begin{array}{c}
\varepsilon_{1}\left(b^{\prime}\right)+\varphi_{1}(b)+t \\
\varepsilon_{1}\left(b^{\prime}\right)
\end{array}\right] G^{\jmath}\left(\tilde{f}_{1}^{\varphi_{1}(b)+t} b^{\prime}\right)+(\text { other terms }) .
$$

This implies that $c_{b^{\prime}} \in q \mathbf{A}_{0}, \overline{c_{b^{\prime}}}=c_{b^{\prime}}$, and $c_{b^{\prime}}\left[\begin{array}{c}\varepsilon_{1}\left(b^{\prime}\right)+\varphi_{1}(b)+t \\ \varepsilon_{1}\left(b^{\prime}\right)\end{array}\right] \mathbf{A}$ for all $t=$ $1, \ldots, \varepsilon_{1}\left(b^{\prime}\right)+1$. Now, it suffices to show that $c_{b^{\prime}} \in \mathbf{A}$, which follows from next lemma.

This far, we have proved that $G^{\jmath}(b)=u$, and hence, we have $e_{1} G^{\jmath}(b)=0$ and $\mathbf{U}_{1}^{\jmath} G^{\jmath}(b) \simeq L(\boldsymbol{\lambda})$. Then, for all $n=1, \ldots, \boldsymbol{\lambda}_{0}-\boldsymbol{\lambda}_{-1}$, we have

$$
f_{1}^{(n)} G^{\jmath}(b)=f_{1}^{(n)} u=\widetilde{f}_{1}^{n} u .
$$

The left-hand side belongs to $M_{\mathbf{A}}$, while the right-hand side belongs to $\mathcal{L}$. Moreover, we have $\psi_{M}^{\jmath}\left(f_{1}^{(n)} G^{\jmath}(b)\right)=f_{1}^{(n)} G^{\jmath}(b)$, and $\widetilde{f}_{1}^{n} u+q \mathcal{L}=\widetilde{f}_{1}^{n} b$. This implies that $f_{1}^{(n)} G^{\jmath}(b)=G^{\jmath}\left(\widetilde{f}_{1}^{n} b\right)$. Thus, the proof completes.

Lemma 10.1.1. Let $A \in \mathbb{Q}(p, q), m \geq n \in \mathbb{Z}_{\geq 0}$. Suppose that $A\left[\begin{array}{c}m+t \\ n\end{array}\right] \in \mathbf{A}$ for all $t=1, \ldots, n+1$. Then, we have $A \in \mathbf{A}$.

Proof. Let us write $A=B / C$ for some $B, C \in \mathbf{A}_{0} \cap \mathbf{A}$ that are coprime. By the hypothesis, $C$ is a common devisor of $\left[\begin{array}{c}m+t \\ n\end{array}\right], t=1, \ldots, n+1$. Hence, it suffices to show that the greatest common divisor of them in $\mathbb{Z}[q]$ is equal to 1 . This is equivalent to say that the greatest common divisor of $a_{t}:=[m+t][m+t-$ $1] \cdots[m+t-n+1], t=1, \ldots, n+1$ is equal to $[n]$ !. Since $[l]=q^{-l} \prod_{1 \neq d \mid l} \Phi_{d}$, where $\Phi_{d}=\Phi_{d}\left(q^{2}\right)$ denotes the $d$-th cyclotomic polynomial in variable $q^{2}$, we have

$$
b_{t}:=q^{n(m+t)-\frac{n(n-1)}{2}} a_{t}=\prod_{l=0}^{n-1} \prod_{1 \neq d \mid(m+t-l)} \Phi_{d},
$$

which is the irreducible decomposition of $b_{t}$ in $\mathbb{Z}\left[q^{2}\right]$. Then, we have

$$
b_{t}=\prod_{d \geq 2} \Phi_{d}^{m_{d, t}}, \text { where } m_{d, t}:=|\{0 \leq l \leq n-1|d|(m+t-l)\}|,
$$

and hence,

$$
\underset{1 \leq t \leq n+1}{\operatorname{gcd}}\left(b_{t}\right)=\prod_{d \geq 2} \Phi_{d}^{\min _{1 \leq t \leq n+1}\left(m_{d, t}\right)} .
$$

We prove that $\min _{1 \leq t \leq n+1}\left(m_{d, t}\right)=\left\lfloor\frac{n}{d}\right\rfloor$ for all $d$. It is clear that $m_{d, t} \geq\left\lfloor\frac{n}{d}\right\rfloor$ for all $t$ since $\left\{m+t, m+t-1, \ldots, m+t-\left(\left\lfloor\frac{n}{d}\right\rfloor d-1\right)\right\}$ contains exactly $\left\lfloor\frac{n}{d}\right\rfloor$ integers divisible by $d$. If $\min _{1 \leq t \leq n+1}\left(m_{d, t}\right)>\left\lfloor\frac{n}{d}\right\rfloor$, then $\left\{m+t-\left\lfloor\frac{n}{d}\right\rfloor d, m+t-\left(\left\lfloor\frac{n}{d}\right\rfloor d+\right.\right.$ $1), \ldots, m+t-(n-1)\}$ contains at least one multiple of $d$ for all $t$. Then, for $t=1$, there exists $l_{1} \in\left\{\left\lfloor\frac{n}{d}\right\rfloor d,\left\lfloor\frac{n}{d}\right\rfloor d+1, \ldots, n-1\right\}$ such that $m+1-\left(\left\lfloor\frac{n}{d}\right\rfloor d+l_{1}\right) \in d \mathbb{Z}$. Set $t^{\prime}:=n-l_{1}+1$, and consider the integers

$$
m+t^{\prime}-\left\lfloor\frac{n}{d}\right\rfloor d, m+t^{\prime}-\left(\left\lfloor\frac{n}{d}\right\rfloor d+1\right), \ldots, m+t^{\prime}-(n-1)=\left(m+1-l_{1}\right)+1 .
$$

These are $\left(n-\left\lfloor\frac{n}{d}\right\rfloor d\right)$ consecutive integers with $\left(m+1-l_{1}\right)+1=1$ modulo $d$. Since $n-\left\lfloor\frac{n}{d}\right\rfloor d<d$, they have no multiples of $d$. Hence, we have $\min _{1 \leq t \leq n+1}\left(m_{d, t}\right)=\left\lfloor\frac{n}{d}\right\rfloor$ 
for all $d \geq 2$. Thus, we obtain

$$
\underset{1 \leq t \leq n+1}{\operatorname{gcd}}\left(b_{t}\right)=\prod_{d \geq 2} \Phi_{d}^{\left\lfloor\frac{n}{d}\right\rfloor}=\prod_{d=2}^{n} \Phi_{d}^{\left\lfloor\frac{n}{d}\right\rfloor}=\prod_{l=2}^{n}\left(\prod_{1 \neq d^{\prime} \mid l} \Phi_{d^{\prime}}\right)=\prod_{l=2}^{n}[l]=[n] ! .
$$

This proves the lemma.

10.2. The $r \geq 2$ case. Now, we are ready to prove Theorem 9.3.6 by induction on $r$.

When $r=1$, we have already completed the proof. Let $r \geq 2$ and assume that the assertions hold for all $r^{\prime}<r$.

Lemma 10.2.1. Let $\boldsymbol{\lambda} \in P^{J}(M)$ be a maximal element, $b \in \mathcal{B}$ such that $I^{\jmath}(b)=\boldsymbol{\lambda}$ and $\widetilde{e}_{i} b=0$ for all $i \in \mathbb{I}^{J}$. Suppose the following:

(1) There exists a homomorphism $\xi: L(\boldsymbol{\lambda}) \rightarrow M$ of $\mathbf{U}^{\jmath}$-modules such that $\xi\left(G_{\text {low }}^{J}\left(T_{b^{\prime}}^{J}\right)\right)=G^{\jmath}\left(b^{\prime}\right)$ for all $b^{\prime} \in C^{\jmath}(b)$ which is strongly connected to some $b^{\prime \prime} \in C^{\jmath}(b)$ with $\mathrm{wt}^{\jmath}(b)<^{\jmath} \mathrm{wt}^{\jmath}\left(b^{\prime \prime}\right)$.

(2) $\xi$ commutes with the $\psi^{\mathrm{J}}$-involutions on $L(\boldsymbol{\lambda})$ and $M$.

(3) $\left[\xi\left(G_{\text {low }}^{\jmath}\left(T_{b}^{\jmath}\right)\right): G^{\jmath}(b)\right]=1$.

Then, we have

$$
\xi\left(G_{\text {low }}^{J}\left(T_{b}^{J}\right)\right)=G^{J}(b)+\sum_{\substack{b^{\prime} \in \mathcal{B} \backslash\{b\} \\ T b^{\prime}=T_{b}^{J}}} c_{b^{\prime}} G^{J}\left(b^{\prime}\right)+\sum_{\substack{b^{\prime \prime} \in C^{J}\left(b^{\prime}\right), c_{b^{\prime}} \neq 0 \\ s\left(b^{\prime \prime}\right)<s\left(b^{\prime}\right)}} c_{b^{\prime \prime}} G^{J}\left(b^{\prime \prime}\right) .
$$

for some $c_{b^{\prime}}, c_{b^{\prime \prime}} \in \mathbf{A}_{0}$.

Proof. Since $\mathbf{U}^{\jmath}$-module homomorphisms preserve $\}$-crystal lattices, we have $\xi\left(G_{\text {low }}^{J}\left(T_{b}^{J}\right)\right) \in \mathcal{L}$, and $\xi\left(G_{\text {low }}^{J}\left(T_{b}^{J}\right)\right)+q \mathcal{L}=b$. Let us write

$$
\xi\left(G_{\text {low }}^{\jmath}\left(T_{b}^{\jmath}\right)\right)=G^{\jmath}(b)+\sum_{b^{\prime} \in \mathcal{B} \backslash\{b\}} c_{b^{\prime}} G^{\jmath}\left(b^{\prime}\right)
$$

for some $c_{b^{\prime}} \in q \mathbf{A}_{0}$. Also, since $\xi$ commutes with $\psi^{J}$-involutions, we have $\overline{c_{b}}=c_{b}$, $\overline{c_{b^{\prime}}}=c_{b^{\prime}}$. We claim the following: if $b^{\prime} \in \mathcal{B} \backslash\{b\}$ satisfies

(†) $\quad c_{b^{\prime}} \neq 0$ and $s\left(b^{\prime}\right)$ is maximal among $\left\{s\left(b^{\prime \prime}\right) \mid b^{\prime \prime} \in \mathcal{B} \backslash\{b\}\right.$ and $\left.c_{b^{\prime \prime}} \neq 0\right\}$,

then $T_{b^{\prime}}^{\jmath}(-i) \geq \boldsymbol{\lambda}_{-i}$ for all $i=0,1, \ldots, r$. By the case $r=1$, we have $I_{1}^{\jmath}\left(b^{\prime}\right) \succeq I_{1}^{\jmath}(b)$, which implies $T_{b^{\prime}}^{J}(0)=T_{b}^{J}(0)=\boldsymbol{\lambda}_{0}$, and $T_{b^{\prime}}^{J}(-1) \geq T_{b}^{J}(-1)=\boldsymbol{\lambda}_{-1}$. We proceed by induction on $i$. Assume that $i \geq 2$, and that $T_{b^{\prime}}^{\jmath}(-(i-1)) \geq \boldsymbol{\lambda}_{-(i-1)}$ for all $b^{\prime}$ satisfying $(\dagger)$. Suppose that there exists $b^{\prime}$ satisfying $(\dagger)$ such that $T_{b^{\prime}}^{j}(-i)<\boldsymbol{\lambda}_{-i}$. Let $b^{\prime \prime} \in \mathcal{B} \backslash\{b\}$ be such that $s\left(b^{\prime \prime}\right)=s\left(b^{\prime}\right)$ and $\varphi_{-b^{\prime \prime}}$ is minimal among such elements. Recall that $s\left(b^{\prime \prime}\right)=s\left(b^{\prime}\right)$ implies $\operatorname{wt}\left(b^{\prime \prime}\right)=\operatorname{wt}\left(b^{\prime}\right)$, and hence, $T_{b^{\prime \prime}}^{J}(-i)=$ $T_{b^{\prime}}^{J}(-i)<\boldsymbol{\lambda}_{-i}$. Then, we have

$$
\varepsilon_{-\underline{i}}\left(b^{\prime \prime}\right)=\varphi_{-\underline{i}}\left(b^{\prime \prime}\right)+T_{b^{\prime \prime}}^{J}(-(i-1))-T_{b^{\prime \prime}}^{J}(-i)>T_{b^{\prime \prime}}^{J}(-(i-1))-\lambda_{-i}+\varphi_{-\underline{i}}\left(b^{\prime \prime}\right) .
$$

By the minimality of $\varphi_{-\underline{i}}\left(b^{\prime \prime}\right)$, it holds that

$$
\left[f_{i}^{(t)} \sum_{b^{\prime} \in \mathcal{B} \backslash\{b\}} c_{b^{\prime \prime}} G^{J}\left(b^{\prime}\right): G^{J}\left(\tilde{f}_{i}^{t} b^{\prime \prime}\right)\right]=c_{b^{\prime}}\left[\begin{array}{c}
t \\
\varphi_{-\underline{i}}\left(b^{\prime \prime}\right)
\end{array}\right] \neq 0
$$

for all $T_{b^{\prime \prime}}^{J}(-(i-1))-\boldsymbol{\lambda}_{-i}+1 \leq t \leq T_{b^{\prime \prime}}^{J}(-(i-1))-\boldsymbol{\lambda}_{-i}+\varphi_{-\underline{i}}\left(b^{\prime \prime}\right)+1$. On the other hand, $f_{i}^{(t)} G_{\text {low }}^{J}\left(T_{b}^{J}\right)$ is the sum of $G_{\text {low }}^{J}\left(T_{\tilde{f}_{i}^{t} b}^{J}\right)$ and an A-linear combination of 
$G_{\text {low }}^{\jmath}\left(T_{\widehat{b}}^{\jmath}\right)$ such that $\widehat{b} \in C^{\jmath}\left(b_{t}\right)$ is strongly connected to $b^{\prime \prime \prime} \in C^{\jmath}\left(b_{t}\right)$ with $\operatorname{wt}^{\jmath}(b)<^{\jmath}$ $\mathrm{wt}^{J}\left(b^{\prime \prime \prime}\right)$. Hence, we have

$$
\begin{aligned}
\xi\left(G_{\text {low }}^{J}\left(T_{\widetilde{f}_{i}^{t} b}^{J}\right)\right) & =f_{i}^{(t)} \xi\left(G_{\text {low }}^{\jmath}\left(T_{b}^{\jmath}\right)\right)+\sum_{\widehat{b}} a_{\widehat{b}} G^{\jmath}(\widehat{b}) \\
& =f_{i}^{(t)} G^{\jmath}(b)+f_{i}^{(t)} \sum_{b^{\prime} \in \mathcal{B} \backslash\{b\}} c_{b^{\prime}} G^{\jmath}\left(b^{\prime}\right)+\sum_{\widehat{b}} a_{\widehat{b}} G^{\jmath}(\widehat{b})
\end{aligned}
$$

for some $a_{\widehat{b}} \in \mathbf{A}$. Here, note that we have $\widetilde{e}_{j} \widetilde{f}_{i}^{t} b=0$ for all $j=1, \ldots, i-1$, $\left[\xi\left(G_{\text {low }}^{\jmath}\left(T_{\widetilde{f}_{i}^{t} b}^{\jmath}\right)\right): G^{\jmath}\left(\widetilde{f}_{i}^{t} b\right)\right]=1$, and $s\left(\widetilde{f}_{i}^{t} b^{\prime \prime}\right)$ is maximal among $\left\{s\left(b^{\prime \prime \prime}\right) \mid b^{\prime \prime \prime} \neq\right.$ $\widetilde{f}_{i}^{t} b$ and $\left.\left[\xi\left(G_{\text {low }}^{\jmath}\left(T_{\tilde{f}_{i}^{t} b}^{\jmath}\right)\right): G^{\jmath}\left(b^{\prime \prime \prime}\right)\right] \neq 0\right\}$. Then, by our induction hypothesis on $i$, we obtain that $T_{\widetilde{f}_{i}^{t} b^{\prime \prime}}^{j}(-(i-1)) \geq T_{\widetilde{f}_{i}^{t} b}^{j}(-(i-1))=\boldsymbol{\lambda}_{-i}$, which is a contradiction since $t \geq T_{b^{\prime \prime}}^{J}(-(i-1))-\boldsymbol{\lambda}_{-i}+1$. Hence we must have $\left[\xi\left(G_{\text {low }}^{J}\left(T_{\widetilde{f}_{i}^{t} b}^{J}\right)\right): G^{\jmath}\left(\widetilde{f}_{i}^{t} b^{\prime \prime}\right)\right]=0$. Since

$$
\left[\xi\left(G_{\text {low }}^{\jmath}\left(T_{\widetilde{f}_{i}^{t} b}^{\jmath}\right)\right): G^{\jmath}\left(\widetilde{f}_{i}^{t} b^{\prime \prime}\right)\right]=c_{b^{\prime \prime}}\left[\begin{array}{c}
t \\
\varphi_{-\underline{i}}\left(b^{\prime \prime}\right)
\end{array}\right]+\left[f_{i}^{(t)} G^{\jmath}(b): G^{\jmath}\left(\widetilde{f}_{i}^{t} b^{\prime \prime}\right)\right]+a_{\widetilde{f}_{i}^{t} b^{\prime \prime}},
$$

and the second and the third term of the right-hand side lies in $\mathbf{A}$, we obtain

$$
c_{b^{\prime \prime}}\left[\begin{array}{c}
t \\
\varphi_{-\underline{i}}\left(b^{\prime \prime}\right)
\end{array}\right] \in \mathbf{A}
$$

for all $T_{b^{\prime \prime}}^{J}(-(i-1))-\boldsymbol{\lambda}_{-i}+1 \leq t \leq T_{b^{\prime \prime}}^{J}(-(i-1))-\boldsymbol{\lambda}_{-i}+\varphi_{-\underline{i}}\left(b^{\prime \prime}\right)+1$. By Lemma 10.1.1 this implies $c_{b^{\prime \prime}}=0$.

This far, we have proved that if $b^{\prime} \in \mathcal{B} \backslash\{b\}$ satisfies $(\dagger)$, then we have $T_{b^{\prime}}^{J}(-i) \geq$ $\boldsymbol{\lambda}_{-i}$ for all $i \in\{0,1, \ldots, r\}$. In particular, we have $\mathbb{I}^{J}\left(b^{\prime}\right)=\boldsymbol{\lambda}$ for such $b^{\prime}$ (since $\boldsymbol{\lambda}$ is maximal in $\left.P^{\jmath}(M)\right)$. In this case, the condition $T_{b^{\prime}}^{\jmath}(-i) \geq \boldsymbol{\lambda}_{-i}$ for all $i$ forces $b^{\prime}$ to satisfy that $T_{b^{\prime}}^{\jmath}=T_{b}^{\jmath}$. Hence, we have

$$
\xi\left(G_{\text {low }}^{J}\left(T_{b}^{J}\right)\right)=G^{J}(b)+\sum_{\substack{b^{\prime} \in \mathcal{B} \backslash\{b\} \\ T_{b^{\prime}}^{J}=T_{b}^{J}}} c_{b^{\prime}} G^{J}\left(b^{\prime}\right)+\sum_{\substack{b^{\prime \prime} \in C^{J}\left(b^{\prime}\right), c_{b^{\prime} \neq 0} \\ s\left(b^{\prime \prime}\right)<s\left(b^{\prime}\right)}} c_{b^{\prime \prime}} G^{J}\left(b^{\prime \prime}\right),
$$

as desired.

Lemma 10.2.2. Let $\boldsymbol{\lambda} \in P^{\jmath}(M)$ be a maximal element, $j \in \mathbb{I}^{\jmath} \backslash\{1\}, b \in \mathcal{B}$ such that $I^{\jmath}(b)=\lambda, \widetilde{e}_{i} b=0$ for all $i \in \mathbb{I}^{\jmath}$, and $\widetilde{e}_{j^{\prime}}(b) \neq 0$. Suppose the following:

(1) There exists a homomorphism $\xi: L(\boldsymbol{\lambda}) \rightarrow M$ of $\mathbf{U}^{3}$-modules such that $\xi\left(G_{\text {low }}^{\jmath}\left(T_{b^{\prime}}^{\jmath}\right)\right)=G^{\jmath}\left(b^{\prime}\right)$ for all $b^{\prime} \in C^{\jmath}(b)$ which is strongly connected to some $b^{\prime \prime} \in C^{\jmath}(b)$ with $\mathrm{wt}^{\jmath}(b)<{ }^{\jmath} \mathrm{wt}^{\jmath}\left(b^{\prime \prime}\right)$.

(2) $\xi$ commutes with the $\psi^{3}$-involutions on $L(\boldsymbol{\lambda})$ and $M$.

Then, we have

$$
\xi\left(G_{\text {low }}^{J}\left(T_{b}^{J}\right)\right)=G^{J}(b)+\sum_{\substack{b^{\prime} \in \mathcal{B} \backslash\{b\} \\ T_{b^{\prime}}^{J}=T_{b}^{J}}} c_{b^{\prime}} G^{J}\left(b^{\prime}\right)+\sum_{\substack{b^{\prime \prime} \in C^{J}\left(b^{\prime}\right), c_{b^{\prime}} \neq 0 \\ s\left(b^{\prime \prime}\right)<s\left(b^{\prime}\right)}} c_{b^{\prime \prime}} G^{J}\left(b^{\prime \prime}\right) .
$$

for some $c_{b^{\prime}}, c_{b^{\prime \prime}} \in \mathbf{A}_{0}$.

Proof. If we can prove that $c_{b}:=\left[\xi\left(G_{\text {low }}^{\jmath}\left(T_{b}^{\jmath}\right)\right): G^{\jmath}(b)\right]=1$, then the assertion follows from the previous lemma. Hence, we aim to show $c_{b}=1$. 
By the same argument as before, we have

$$
\left[\xi\left(G_{\text {low }}^{J}\left(T_{\widetilde{f}_{i}^{t} b}^{\jmath}\right)\right): G^{J}\left(\widetilde{f}_{i}^{t} b^{\prime \prime}\right)\right]=c_{b^{\prime \prime}}\left[\begin{array}{c}
t \\
\varphi_{-\underline{i}}\left(b^{\prime \prime}\right)
\end{array}\right]+c_{b}\left[f_{i}^{(t)} G^{\jmath}(b): G^{J}\left(\widetilde{f}_{i}^{t} b^{\prime \prime}\right)\right]+a_{\widetilde{f}_{i}^{t} b^{\prime \prime}},
$$

for all $b^{\prime \prime} \in \mathcal{B} \backslash\{b\}$ satisfying $(\dagger)$. Here, let us assume further that $s\left(b^{\prime}\right)>s(b)$. Then, we have $\left[f_{i}^{(t)} G^{\jmath}(b): G^{\jmath}\left(\tilde{f}_{i}^{t} b^{\prime \prime}\right)\right]=0$ since $f_{i}^{(t)} G^{\jmath}(b)$ is a linear combination of $G^{\jmath}(\tilde{b})$ with $s(\widetilde{b}) \leq s(b)+t \alpha_{-\underline{i}}<s\left(b^{\prime \prime}\right)+t \alpha_{-\underline{i}}=s\left(\widetilde{f}_{i}^{t} b^{\prime \prime}\right)$. Hence, we have $c_{b^{\prime \prime}}\left[\begin{array}{c}t \\ \varphi_{-\underline{i}}\left(b^{\prime \prime}\right)\end{array}\right] \in \mathbf{A}$, and therefore, $c_{b^{\prime \prime}}=0$ by Lemma 10.1.1. In particular, we obtain that $s(b)$ is maximal. Then, we have

$$
\left[e_{j}\left(c_{b} G^{J}(b)+\sum c_{b^{\prime}} G^{\jmath}\left(b^{\prime}\right)\right): G^{J}\left(\widetilde{e}_{j^{\prime}} b\right)\right]=c_{b}\left[\varphi_{\underline{j}}(b)+1\right]
$$

On the other hand, since $\left[e_{j} G_{\text {low }}^{J}\left(T_{b}^{j}\right): G_{\text {low }}^{J}\left(\widetilde{e}_{j^{\prime}} b\right)\right]=\left[\varphi_{\underline{j}}(b)+1\right]$, we have

$$
\left[e_{j}\left(c_{b} G^{\jmath}(b)+\sum c_{b^{\prime}} G^{\jmath}\left(b^{\prime}\right)\right): G^{\jmath}\left(\widetilde{e}_{j^{\prime}} b\right)\right]=\left[\varphi_{\underline{j}}(b)+1\right]
$$

and hence, $c_{b}=1$, as desired.

We prove Theorem 9.3 .6 by descending induction (with respect to $\preceq$ ) on $\boldsymbol{\lambda}$. As in the $r=1$ case, we may assume that $\boldsymbol{\lambda}$ is maximal among $P^{J}(M)$. Then, in order to complete the proof, we have to show the following:

(1) $I_{\boldsymbol{\lambda}}(M)$ has a basis $\left\{G^{\jmath}(b) \mid I^{\jmath}(b)=\boldsymbol{\lambda}\right\}$.

(2) There exists an isomorphism $\xi: L(\boldsymbol{\lambda})^{\oplus m_{\boldsymbol{\lambda}}} \rightarrow I_{\boldsymbol{\lambda}}(M)$ of $\mathbf{U}^{\jmath}$-modules which sends the $\mathrm{j}$-global basis elements of $L(\boldsymbol{\lambda})^{\oplus m_{\boldsymbol{\lambda}}}$ to those of $I_{\boldsymbol{\lambda}}(M)$, where $m_{\boldsymbol{\lambda}}$ denotes the multiplicity of $L(\boldsymbol{\lambda})$ in $M$.

Let $b_{1}, \ldots, b_{m_{\boldsymbol{\lambda}}} \in \mathcal{B}$ and $u_{1}, \ldots, u_{m_{\boldsymbol{\lambda}}} \in \mathcal{L}$ be distinct highest weight vectors of type $\boldsymbol{\lambda}$ with $u_{t}+q \mathcal{L}=b_{t}, t=1, \ldots, m_{\boldsymbol{\lambda}}$. By retaking the $u_{t}$ 's if necessary, we may assume that $\left[u_{t}: G^{\jmath}\left(b_{u}\right)\right]=\delta_{t, u}$ for all $t, u$. Let $\xi_{t}: L(\boldsymbol{\lambda}) \rightarrow M$ be the $\mathbf{U}^{3}$-homomorphism which sends $v_{\boldsymbol{\lambda}}$ to $u_{t}$.

Lemma 10.2.3. We have $\xi_{t}\left(G_{\text {low }}^{J}\left(T_{b_{t}}^{J}\right)\right)=G^{\jmath}\left(b_{t}\right)$ for all $t=1, \ldots, m_{\boldsymbol{\lambda}}$.

Proof. By the setting above, we can write

$$
\xi_{t}\left(G_{\text {low }}^{J}\left(T_{b_{t}}^{\jmath}\right)\right)=u_{t}=G^{\jmath}\left(b_{t}\right)+\sum_{\substack{b^{\prime} \\ I^{\prime}\left(b^{\prime}\right) \nsucceq \boldsymbol{\lambda}}} c_{b^{\prime}} G^{\jmath}\left(b^{\prime}\right), \quad c_{b^{\prime}} \in q \mathbf{A}_{0} .
$$

Then, we can apply Lemma 10.2.1 to obtain $\xi_{t}\left(G_{\text {low }}^{J}\left(T_{b_{t}}^{J}\right)\right)=G^{\jmath}\left(b_{t}\right)$ as desired.

In order to complete the proof, it suffices to prove the following: For each $t=1, \ldots, m_{\boldsymbol{\lambda}}$ and $b \in C^{\jmath}\left(b_{t}\right)$, we have $\xi_{t}\left(G_{\text {low }}^{\jmath}\left(T_{b}^{\jmath}\right)\right)=G^{\jmath}(b)$. We prove this statement by descending induction on $\mathrm{wt}^{\jmath}(b)$ and $I_{r-1}^{\jmath}(b)$. When $\mathrm{wt}^{\jmath}(b)$ is maximal, it must hold that $b=b_{t}$, and in this case, we have already shown that $\xi_{t}\left(G_{\text {low }}^{J}\left(T_{b_{t}}^{\jmath}\right)\right)=G^{J}\left(b_{t}\right)$. Suppose that $\mathrm{wt}^{\jmath}(b)<^{\jmath} \mathrm{wt}^{\jmath}\left(b_{t}\right)$, and the statement holds for all $b^{\prime} \in \bigsqcup_{t=1}^{m_{\lambda}} C^{\jmath}\left(b_{t}\right)$ such that $\mathrm{wt}^{\jmath}\left(b^{\prime}\right)^{\jmath}>\mathrm{wt}^{\jmath}(b)$ or $\mathrm{wt}^{\jmath}\left(b^{\prime}\right)=\mathrm{wt}^{\jmath}(b)$ and $I_{r-1}^{J}\left(b^{\prime}\right) \succ I_{r-1}^{J}(b)$. In this case, since $b$ is not a $\mathbf{U}^{\jmath}$-highest weight vector, the exists $i \in \overline{\mathbb{I}^{\jmath}}$ such that $\widetilde{e}_{i} b \neq 0$.

Lemma 10.2.4. Suppose there exists $i \in \mathbb{I}^{\jmath}$ such that $\widetilde{e}_{i} b \neq 0$. Then, the statement holds. 
Proof. Set $b^{\prime}:=\widetilde{e}_{i}^{\varepsilon_{i}(b)} b$. We prove the lemma by descending induction on $\varepsilon_{i}\left(b^{\prime}\right)$. Since $\mathrm{wt}^{\jmath}\left(b^{\prime}\right)>\mathrm{wt}^{\jmath}(b)$, we have $G^{\jmath}\left(b^{\prime}\right)=\xi_{t}\left(G_{\text {low }}^{\jmath}\left(T_{b^{\prime}}^{\jmath}\right)\right) \in \mathbf{U}^{\jmath} G^{\jmath}\left(b_{i}\right)$. We know that $G^{\jmath}(b)$ (resp., $G_{\text {low }}^{J}\left(T_{b}^{\jmath}\right)$ ) is the sum of $\widetilde{f}_{i}^{\left(\varepsilon_{i}(b)\right)} G^{\jmath}\left(b^{\prime}\right)$ (resp., $\widetilde{f}_{i}^{\left(\varepsilon_{i}(b)\right)} G_{\text {low }}^{J}\left(T_{b^{\prime}}^{\jmath}\right)$ ) and a $q \mathbb{Q}[q]$-linear combination of $G^{\jmath}\left(b^{\prime \prime}\right)$ (resp., $\left.G_{\text {low }}^{J}\left(T_{b^{\prime \prime}}^{J}\right)\right)$ with $\operatorname{wt}^{\jmath}\left(b^{\prime \prime}\right)=\mathrm{wt}^{\jmath}(b)$ and $\varepsilon_{i}\left(b^{\prime \prime}\right)>\varepsilon_{i}(b)$. By our induction hypothesis, $G^{\jmath}(b)-\xi_{t}\left(G_{\text {low }}^{\jmath}(b)\right)$ is a $q \mathbb{Q}[q]$-linear combination of $G^{\jmath}\left(b^{\prime \prime}\right)$ 's, and is $\psi_{M^{J}}^{J}$-invariant. Such a vector must be zero, and hence, we obtain $G^{\jmath}(b)=\xi_{t}\left(G_{\text {low }}^{J}(b)\right)$.

Lemma 10.2.5. Suppose there exists $j \in \mathbb{I}^{\jmath} \backslash\{1\}$ such that $\widetilde{e}_{j^{\prime}} b \neq 0$ and $\widetilde{e}_{i} b=0$ for all $i \in \mathbb{I}^{\jmath}$. Then, the statement holds.

Proof. Apply Lemma 10.2.2.

Now, one can complete the proof by combining Lemma 10.2.3 10.2.5 since each $b \in \mathcal{B}$ with $I^{J}(b)=\boldsymbol{\lambda}$ is connected to $b_{t}$ for some $t=1, \ldots, m_{\boldsymbol{\lambda}}$.

\section{REFERENCES}

[BW18a] Huanchen Bao and Weiqiang Wang, A new approach to Kazhdan-Lusztig theory of type $B$ via quantum symmetric pairs (English, with English and French summaries), Astérisque 402 (2018), vii+134. MR 3864017

[BW18b] Huanchen Bao and Weiqiang Wang, Canonical bases arising from quantum symmetric pairs, Invent. Math. 213 (2018), no. 3, 1099-1177, DOI 10.1007/s00222-018-0801-5. MR.3842062

[BWW18] Huanchen Bao, Weiqiang Wang, and Hideya Watanabe, Multiparameter quantum Schur duality of type B, Proc. Amer. Math. Soc. 146 (2018), no. 8, 3203-3216, DOI 10.1090/proc/13749. MR3803649

[BWW20] Huanchen Bao, Weiqiang Wang, and Hideya Watanabe, Canonical bases for tensor products and super Kazhdan-Lusztig theory, J. Pure Appl. Algebra 224 (2020), no. 8, 106347, 9, DOI 10.1016/j.jpaa.2020.106347. MR4074585

[BI03] Cédric Bonnafé and Lacrimioara Iancu, Left cells in type $B_{n}$ with unequal parameters, Represent. Theory 7 (2003), 587-609, DOI 10.1090/S1088-4165-03-00188-2. MR2017068

[DDPW08] Bangming Deng, Jie Du, Brian Parshall, and Jianpan Wang, Finite dimensional algebras and quantum groups, Mathematical Surveys and Monographs, vol. 150, American Mathematical Society, Providence, RI, 2008. MR2457938

[Deo87] Vinay V. Deodhar, On some geometric aspects of Bruhat orderings. II. The parabolic analogue of Kazhdan-Lusztig polynomials, J. Algebra 111 (1987), no. 2, 483-506, DOI 10.1016/0021-8693(87)90232-8. MR916182

[HK02] Jin Hong and Seok-Jin Kang, Introduction to quantum groups and crystal bases, Graduate Studies in Mathematics, vol. 42, American Mathematical Society, Providence, RI, 2002. MR1881971

[K90] Masaki Kashiwara, Crystalizing the q-analogue of universal enveloping algebras, Comm. Math. Phys. 133 (1990), no. 2, 249-260. MR.1090425

[K93] Masaki Kashiwara, Global crystal bases of quantum groups, Duke Math. J. 69 (1993), no. 2, 455-485, DOI 10.1215/S0012-7094-93-06920-7. MR 1203234

[K02] Masaki Kashiwara, On level-zero representations of quantized affine algebras, Duke Math. J. 112 (2002), no. 1, 117-175, DOI 10.1215/S0012-9074-02-11214-9. MR.1890649

[KL79] David Kazhdan and George Lusztig, Representations of Coxeter groups and Hecke algebras, Invent. Math. 53 (1979), no. 2, 165-184, DOI 10.1007/BF01390031. MR.560412

[LL19] C.-J. Lai and L. Luo, Schur algebras and quantum symmetric pairs with unequal parameters, arXiv:1808.00938v3

[Le99] Gail Letzter, Symmetric pairs for quantized enveloping algebras, J. Algebra 220 (1999), no. 2, 729-767, DOI 10.1006/jabr.1999.8015. MR.1717368 
[L90a] G. Lusztig, Canonical bases arising from quantized enveloping algebras, J. Amer. Math. Soc. 3 (1990), no. 2, 447-498, DOI 10.2307/1990961. MR.1035415

[L90b] George Lusztig, Quantum groups at roots of 1, Geom. Dedicata 35 (1990), no. 1-3, 89-113, DOI 10.1007/BF00147341. MR.1066560

[L10] George Lusztig, Introduction to quantum groups, Modern Birkhäuser Classics, Birkhäuser/Springer, New York, 2010. Reprint of the 1994 edition. MR2759715

[L03] G. Lusztig, Hecke algebras with unequal parameters, CRM Monograph Series, vol. 18, American Mathematical Society, Providence, RI, 2003. MR 1974442

[W17] H. Watanabe, Crystal basis theory for a quantum symmetric pair $\left(\mathbf{U}, \mathbf{U}^{\jmath}\right)$, to appear in Int. Math. Res. Not., arXiv:1704.01277

[X94] Nan Hua Xi, Representations of affine Hecke algebras, Lecture Notes in Mathematics, vol. 1587, Springer-Verlag, Berlin, 1994. MR1320509

Research Institute for Mathematical Sciences, Kyoto University, Kyoto 606-8052, JAPAN

Email address: hideya@kurims.kyoto-u.ac.jp 